\title{
The Longitudinal Index Theorem for Foliations
}

By

A. CONNES* and G. SKANDALIS**

Ingtroduction

In this paper, we use the bivariant $\mathbb{K}$ theory of Kasparov ([19]) as a basic tool to prove the $\mathbb{K}$-theoretical version of the index theorem for longitudinal elliptic differential operators for foliations which is stated as a problem in [10], Section 10. When the foliation is by the fibers of a fibration, this theorem reduces to the Atiyah-Singer index theorem for families ([2], Theorem 3.1). It implies the index theorem for measured foliations ([9], Theorem, p.136) and unlike the latter makes sense for arbitrary foliations, not necessarily gifted with a holonomy invariant transverse measure.

The index in the Atiyah Singer theorem for families ([2]) is an element of the $K$-theory $K^{0}(B)$ of the base space of the fibration. In the case of foliations the base $B$ is the space of leaves of the foliation $(V, F)$. This space of leaves, as a topological space, is often degenerate (if the foliation is minimal there are no nontrivial open sets in $V / F)$. The algebra $C(B)$ of continuous functions on $B$ is replaced by a canonically defined $C^{*}$-algebra: $C^{*}(V, F)$, cf. [9], [10]. The $K$ theory $K_{0}\left(C^{*}(V, F)\right)$ of this $C^{*}$-algebra plays the role of $K^{0}(B)$. In the case of a fibration $C^{*}(V, F)$ is (Morita) equivalent to $C(B)$ so that $K_{0}\left(C^{*}(V, F)\right)=\mathbb{K}^{0}(B)$.

Let $D$ be an elliptic differential operator along the leaves of the foliation $(V, F)$. Since $D$ is elliptic it has an inverse modulo $\mathbb{C}^{*}(V, F)$ hence it gives an element $\operatorname{Ind}_{a}(D)$ of $\mathbb{K}_{0}\left(C^{*}(V, \mathbb{F})\right.$ ). Let us now describe the topological index. Let $i$ be an auxiliary imbedding of the manifold $V$ in $\mathbb{R}^{2 n}$. Let $N$ be the total space of the normal bundle to the leaves: $N_{x}=\left(i_{*}\left(F_{x}\right)\right)^{\perp} \subset \mathbb{R}^{2 n}$. Let us foliate $\tilde{V}=V \times \mathbb{R}^{2 n}$ by $\widetilde{F}, \widetilde{F}_{(x, t)}=\mathbb{F}_{x} \times\{0\}$, so that the leaves of $(\tilde{V}, \widetilde{F})$ are just $\widetilde{L}=\mathbb{L} \times\{t\}$,

Communicated by H. Araki, August 16, 1983.

* Institut des Hautes Etudes Scientifiques, 35 route de Charires, 91440 Bures-sur-Yvette, France.

** Universite P. et M. Curie, Tour 45-46, HUER 48, 4 Place Jussieu, Paris, France. 
where $L$ is a leaf of $(V, F)$ and $t \in \mathbb{R}^{2 n}$. The map $(x, \xi) \rightarrow(x, i(x)+\xi)$ turns an open neighborhood of the 0-section in $N$ into an open transversal $T$ of the foliation $(\tilde{V}, \widetilde{F})$. For a suitable open neighborhood $\Omega$ of $T$ in $\tilde{V}$, the $C^{*}$-algebra $C^{*}(\Omega, \widetilde{F})$ of the restriction of $\widetilde{F}$ to $\Omega$ is (Morita) equivalent to $C_{0}(T)$, hence the inclusion $C^{*}(\Omega, \widetilde{F}) \subset C^{*}(\widetilde{V}, \widetilde{F})$ yields a $K$-theory map: $K^{0}(N) \rightarrow K_{0}\left(C^{*}(\tilde{V}, \widetilde{F})\right)$. Since $C^{*}(\tilde{V}, \widetilde{F})=C^{*}(V, F) \otimes C_{0}\left(\mathbb{R}^{2 n}\right)$, one has, by Bott periodicity, the equality $K_{0}\left(C^{*}(\tilde{V}, \widetilde{F})\right)=K_{0}\left(C^{*}(V, F)\right)$.

Using the Thom isomorphism $K^{0}\left(F^{*}\right)$ is identified with $K^{0}(N)$ so that one gets by the above construction, the topological index:

$$
\operatorname{Ind}_{t}: \mathbb{K}^{0}\left(F^{*}\right) \rightarrow K_{0}\left(C^{*}(V, F)\right) .
$$

Our main result is the equality: $\operatorname{Ind}_{a}(D)=\operatorname{Ind}_{t}\left(\left[\sigma_{D}\right]\right)$ where $\sigma_{D}$ is the longitudinal symbol of $D$ and $\left[\sigma_{D}\right]$ is its class in $K^{0}\left(F^{*}\right)$.

In the first section, we formalize the elliptic pseudo-differential calculus for families of operators on $X$ indexed by $Y$, in terms of the bivariant Kasparov theory. This gives a map of the $K$-theory with compact support $K^{*}\left(T^{*} X \times Y\right)$ to the bivariant group $K K^{*}(X, Y)$. We then compute directly the Kasparov product of two such elements.

In the second section we first recall the definition of [10] of the analytical element $f ! \in \mathbb{K} K(X, Y)$ corresponding to a $\mathbb{K}$-oriented map $f$ from $X$ to $Y$. We then prove that $\left(\mathrm{id}_{X}\right)$ ! is the unit of the ring $K K(X, X)$. Using the computation of Section $\mathbb{I}$, we then prove the equality: $(f \circ g) !=g ! \otimes f !$. Computing $f$ ! in the case of an immersion, one gets that in all cases the map $K(X) \rightarrow K(Y)$ given by $f$ ! coincides with the classical wrong way map in $K$-theory. This statement is an index theorem for morphisms.

In Section III we show that in the context of smooth manifolds the elements of the bivariant $\mathbb{K a s p a r o v}$ group $\mathbb{K} K(C(V), \mathbb{C}(W))$ have a natural interpretation in terms of correspondences between $V$ and $W$. The Kasparov product is then given by a simple fibered product formula whose existence relies on the transversality theorem. It follows then that, in this context, all Kasparov products can be computed in purely geometric term. For instance the Poincaré duality in analytical $K$ theory, is easily derived at the end of Chapter III from simple geometric considerations. As applications we also derive the odd index theorem of Baum-Douglas [6] and Kasparov [17]. As another example we exhibit geometrically the correspondence from a submanifold $W$ (of the manifold $V$ ) to the complement of $W$ in $V$ whose associated analytical element in $K K^{1}(W, V / W)$ is given by the exact sequence of $C^{*}$ algebras: $0 \rightarrow C_{0}(V / W) \rightarrow$ 
$C_{0}(V) \rightarrow C_{0}(W) \rightarrow 0$. In particular, the connecting map from the $\mathbb{K}$ homology of $V / W$ to the $K$ homology of $W$ has again a simple geometric description.

In Section IV we prove the above-mentioned longitudinal index theorem and at the same time the existence of a map $\mu_{:} K_{*, \tau}(B G) \rightarrow \mathbb{K}^{*}(V / F)$ from the geometric group [5], [10] of a foliation $(V, F)$ with graph $G$ to the analytical group. We then illustrate it by a simple example.

Finally, in the appendix we introduce in the general theory of Kasparov a notion of connection which allows to compute Kasparov products without modifying first the $C^{*}$ modules involved. It gives an implicit characterization of the Kasparov product which we use in a crucial manner in our computations throughout the paper. We also give a detailed description of the notion of $\mathbb{K}$ orientation for microbundles which is the natural framework of Section $\mathbb{I}$. All the results of this paper have been announced in [11].

\section{Table of Conterits}

I. The Kasparov product of pseudo-differential families.

III. Wrong way functoriality.

直耳. Composition of correspondences and applications.

$\mathbb{I V}$. The longitudinal index theorem.

$\mathbb{V}$. Appendix $A$. Connections and implicit characterization of Kasparov products.

$\mathbb{V}$. Appendix B. $\mathbb{K}$-orientations of microbundles.

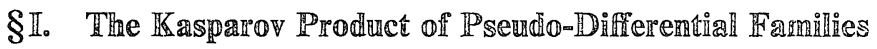

Let $X$ be a smooth manifold and $Y$ a locally compact parameter space. In this section we shall first interpret the construction of continuous families, indexed by $Y$, of pseudo-differential operators on $X$, as yielding a map $\Psi^{*}$ : $K\left(T^{*} X \times Y\right) \rightarrow K K(X, Y)$. Then we compute the Kasparov product of two such families from a formula at the symbol level.

For the simplicity of the statements that follow we will assume that the manifold $X$ is compact. We will indicate in Remarks $(1.5$ (a)) and (1.11 (a)) the minor changes needed in the non compact case.

Denote by $C_{0}(Y)$ the $C^{*}$-algebra of continuous functions vanishing at $\infty$ in $Y$.

Let $\Omega$ be an open subset of $X \times Y$ and $E$ a Hermitian vector bundle over $\Omega$. Let $C_{c}\left(\Omega, E \otimes \Omega^{1 / 2}\right)$ be the space of continuous (1/2 density) sections of $E$ 
with compact support. Let $\mathfrak{S}=\mathfrak{S}(\Omega, E)$ denote the completion of $C_{c}\left(\Omega, E \otimes \Omega^{1 / 2}\right)$ with respect to the $C_{0}(Y)$ valued inner product $\langle\xi, \eta\rangle(y)=\int\langle\xi(x, y), \eta(x, y)\rangle$. Then $\mathfrak{S}$ is a Hilbert $C^{*}$-module ([18], Definition 2$)$ over $C_{0}(Y)$.

1.1. Remarks. 1. We denote here by $\Omega^{1 / 2}$ the bundle of half densities in the $X$ direction. The scalar product $\langle\xi(x, y), \eta(x, y)\rangle$ is a density in the $X$ direction. Hence its integral over $\Omega_{y}=\{x \mid(x, y) \in \Omega\}$ is well defined.

In all this paper we will use half densities without mentioning the bundle they are attached to. Their use will be to give formulae which do not depend upon the choice of a measure in the Lebesgue class.

2. A Hilbert $C^{*}$-module $\mathcal{E}$ over $C_{0}(Y)$ is the space of continuous sections vanishing at $\infty$ of a continuous field $\left(\mathcal{E}_{y}\right)_{y \in X}$ of Hilbert spaces (in the sense of [12], 110$)$. Here we have $\mathfrak{S}(\Omega, E)_{y}=L^{2}\left(\Omega, E_{y}\right)$ where $E_{y}$ is the restriction of $E$ to $\Omega_{y}=\{x \mid(x, y) \in \Omega\}$. Note that the scalar product in $\mathbb{L}^{2}\left(\Omega_{y}, E_{y}\right)$ is canonically defined using half densities.

3. Following [18] and [19], we take all scalar products to be linear in the second variable, antilinear in the first.

We next define the algebra of order 0 pseudo-differential families. It is a subalgebra of the $C^{*}$-algebra $\mathfrak{L}(\mathfrak{S}(\Omega, E))$ of endomorphisms of the Hilbert $C_{0}(Y)$ module $\mathfrak{C}(\Omega, E)$ ([18], Definition 3$)$.

We shall first see the pseudo-differential families as acting on the $C^{\infty, 0}$ sections with compact support of the bundle $E$.

Recall ([2]) that a function $f$ on $X \times Y$ is of class $C^{\infty, 0}$ if the map $y \rightarrow f(\circ, y)$ from $Y$ to $C^{\infty}(X)$ is continuous. The notion of bundle of class $C^{\infty, 0}$ over an open subset $\Omega \subseteq X \times Y$ is defined analogously. If $E$ is such a bundle, $C_{c}^{\infty, 0}(E)$ denotes the space of $C^{\infty, 0}$ sections of $E$, with compact support in $\Omega$ (cf. [2], p. 121-124).

Set $T^{*} \Omega=\left\{(x, \xi, y) /(x, \xi) \in T^{*} X,(x, y) \in \Omega\right\}$. A symbol of order 0 is a function $a \in C^{\infty, 0}\left(T^{*} \Omega, \mathfrak{L}(E)\right)$ which, uniformly on compact subsets of $Y$, has an asymptotic expansion $a \sim \sum_{m=0}^{\infty} \sigma_{m}$ where $\sigma_{m}$ is homogeneous of degree $-m$. To such a symbol one associates a continuous linear map $f \rightarrow P f$ from $C_{c}^{\infty, 0}(\Omega, \mathbb{E} \otimes$ $\left.\Omega^{1 / 2}\right)$ to $C^{\infty, 0}\left(\Omega, E \otimes \Omega^{1 / 2}\right)$ by the usual formula:

$$
(P f)(x, y)=\int \exp \left(i \phi\left(x, x^{\prime}, \xi\right)\right) a(x, y, \xi) \chi\left(x, x^{\prime}, y\right) f\left(x^{\prime}, y\right) d x^{\prime} d \xi^{(*)} .
$$

Here the integral is an oscillating integral, $\phi$ is a phase function and $\chi$ is a

(*) Where $d \xi$ stands for $(2 \pi)^{-\operatorname{dim} x} \times$ Lebesgue measure. 
cut off function associated with the diagonal of $X \times X\left(\chi\left(x, x^{\prime}, y\right) \in \mathfrak{R}\left(E_{x^{\prime}, y} \otimes \Omega_{x^{\prime}}^{1 / 2}\right.\right.$, $\left.E_{x, y} \otimes \Omega_{x}^{1 / 2}\right), \chi(x, x, y)=1_{E x, y}$ cf., for instance [7] or [16]).

As $(P f)(x, y)$ only depends upon the restriction of $f$ to $X \times\{y\}, \mathbb{P}$ is a family $\left(P_{y}\right)_{y \in Y}$ of pseudo-differential operators on $X$. We shall say that the support of $P$ is contained in a closed subset $K$ of $\Omega$, when, for each $y \in Y$, the distribution kernel of $\mathbb{P}_{y}$ has support in $K_{y} \times K_{y}\left(K_{y}=\{x \in X,(x, y) \in K\}\right)$.

It is clear (cf. [24], Theorem 1, p. 243) that a family $P$ as above, with compact support $K \subset \Omega$, extends to an endomorphism (still called $P$ ) of the $\mathbb{C}_{0}(Y)$ module $\mathfrak{S}(\Omega, E)$. Let $\Psi_{0}^{*}(\Omega, E)$ denote the norm closure in $\Omega(S(\Omega, E)$ ) of the *-algebra generated by the above $P$ 's and the ideal $\Re(S(\Omega, E))$ of compact endomorphisms of $\mathfrak{S}(\Omega, E)([18]$, Definition 4$)$.

A bounded section $f \in C_{b}(\Omega, \mathfrak{L}(E))$ determines an endomorphism-still noted $f$-of $\mathcal{S}(\Omega, E)$ by the formula $(f \xi)(x, y)=f(x, y) \xi(x, y)\left(\xi \in \mathbb{C}_{c}(\Omega, \mathbb{E} \otimes\right.$ $\left.\Omega^{1 / 2}\right)$ ). For $P \in \Psi_{0}^{*}(\Omega, E)$ and $f \in C_{b}(\Omega, \Omega(E))$ boih $f P$ and $P f \in \Psi_{0}^{*}(\Omega, E)$. Hence the closed subspace of $\mathfrak{R}(\mathscr{C}(\Omega, E))$ generated by $\Psi_{0}^{*}(\Omega, E)$ and $C_{b}(\Omega, \mathscr{R}(E))$ is a $C^{*}$-algebra $\Psi^{*}(\Omega, E)$.

Every $P \in \Psi^{*}(\Omega, E)$ has a symbol of order $0, \sigma(P)$ which lies in the $C^{*}$. algetra $\mathfrak{S}(\Omega, E)=C_{0}\left(S^{*} \Omega, \Omega(E)\right)+C_{b}\left(\Omega, \Omega(E)\right.$ ) (as subspaces of $C_{b}\left(S^{*} \Omega, \Omega(E)\right)$ ). Here

$$
S^{*} \Omega=\left\{(x, \xi, y) ;(x, y) \in \Omega, \xi \text { is a half line of } \mathbb{T}_{x}^{*}(X)\right\}^{(*)} .
$$

The following proposition is a particular case of [9], p. 138 (cf. [24], Coroliary, p. 265):

1.2. Proposition. The following sequences are exact:

$$
\begin{aligned}
& 0 \rightarrow \mathfrak{S}(\mathfrak{Q}(\Omega, E)) \rightarrow \Psi_{0}^{*}(\Omega, E) \stackrel{\sigma}{\rightarrow} C_{0}\left(S^{*} \Omega, \mathfrak{L}(E)\right) \rightarrow 0 \\
& 0 \rightarrow \Re(\mathfrak{S}(\Omega, E)) \rightarrow \Psi^{*}(\Omega, E) \stackrel{\sigma}{\rightarrow} \mathfrak{S}(\Omega, E) \rightarrow 0 .
\end{aligned}
$$

The construction of the map $\Psi^{*}: K\left(T^{*} X \times Y\right) \rightarrow K K(X, Y)$ follows now in a formal manner. It will be useful to use a rather large class of symbols $\sigma \in \mathbb{K}\left(T^{*}\right.$ $X \times Y$ ) only defined above an open subset $\Omega$ of $X \times Y$ and satisfying:

1.3. Defimition. A Clifford symbol is a triple $(\Omega, E, \sigma)$ where $\Omega$ is an open subset of $X \times Y, E$ is a $\mathbb{Z} / 2$ graded Hermition bundle of class $\mathbb{C}^{\infty, 0}$ over $\Omega$, and $\sigma \in \mathfrak{S}(\Omega, E)$ satisfies:

(a) $\sigma(x, \xi, y)$ is of degree 1 (for the $\mathbb{Z} / 2$ grading of $\mathbb{Q}(E)$ ) for all $(x, \xi, y) \in$

(*) Or $\xi \in T_{\tilde{z}}^{*} X\|\xi\|=1$ for a given Riemannian metric. 
$S^{*} \Omega$

(b) $\sigma=\sigma^{*}, \sigma^{2}=1$,

(c) $\sigma$ has compact support. This means that there exists a compact subset $K$ of $\Omega$ such that $\sigma(x, \xi, y)$ only depends on $(x, y)$ outside $K$.

Such a symbol is called trivial if $\sigma$ is everywhere independent of $\xi$. The $K$-theory obtained from stable homotopy classes of Clifford symbols (modulo the trivial ones) is canonically isomorphic (using the excision map) to the $\mathbb{K}$ theory with compact supports $K\left(T^{*} X \times Y\right.$ ) (cf. [1], §2).

Let $(\Omega, E, \sigma)$ be a Clifford symbol. By Proposition 1.2 , there exists $P=$ $P_{\sigma} \in \Psi^{*}(\Omega, E)$, of degree $1^{(*)}$, such that $\sigma(P)=\sigma$ 。

1.4. Proposition. The pair $\left(\mathfrak{S}(\Omega, E), \mathbb{P}_{\sigma}\right)$ defines an element of $K K(X, Y)$. Moreover its class $\Psi^{*}([\sigma])$ only depends upon the class of $(\Omega, E, \sigma)$ in $K\left(T^{*} X \times Y\right)$.

Proof. That $\mathbb{P}_{\sigma}$ defines an element of $K K(X, Y)$ means that $P_{\sigma}-P_{\sigma}^{*}, P_{\sigma}^{2}-1$ and $\left[f, P_{\sigma}\right] \in \Re(S(\Omega, E)$ ) (for $f \in C(X)$ ). This follows from Proposition 1.2. Note that a homotopy of symbols can be lifted to a homotopy between $\mathbb{K a s p a r o v}$ elements using Proposition 1.2 with $Y \times[0,1]$ instead of $Y$.

1.5. Remarks. (a) Assume that $X$ is not compact. Put

$$
\begin{aligned}
& \mathfrak{\Omega}_{X}(\mathfrak{L}(\Omega, E))=\left\{T \in \mathfrak{L}(\mathfrak{S}), T f \in \mathfrak{R}(\mathfrak{S}), \forall f \in C_{0}(X)\right\} \\
& \Psi_{0, X}^{*}(\Omega, E)=\left\{T \in \mathfrak{L}(\mathfrak{S}), f T \in \Psi_{0}^{*}, \forall f \in C_{0}(X)\right\} \text { and } \\
& \Psi_{X}^{*}(\Omega, E)=\left\{T \in \mathfrak{L}(\mathfrak{S}), f T \in \Psi^{*}(\Omega, E), \forall f \in C_{0}(X)\right\}=\Psi_{0, X}^{*}+C_{b}(\Omega, \mathscr{L}(E)) .
\end{aligned}
$$

Let also:

$$
\begin{aligned}
& C_{0, X}\left(S^{*} \Omega, \mathfrak{R}(E)\right)=\left\{\sigma \in C_{b}\left(S^{*} \Omega, \mathfrak{L}(E)\right), f \sigma \in C_{0}\left(S^{*} \Omega, \mathfrak{L}(E)\right), V f \in C_{0}(X)\right\} \\
& \mathfrak{S}_{X}(\Omega, E)=\left\{\sigma \in C_{b}\left(\mathbb{R}^{*} \Omega, \mathfrak{L}(E)\right), f \sigma \in \mathcal{S}(\Omega, E)\right\}=C_{0, X}\left(S^{*} \Omega, \mathfrak{L}(E)\right)+C_{b}(\Omega, \mathfrak{L}(E)) .
\end{aligned}
$$

One uses instead of Proposition 1.2 the exactness of:

$$
0 \rightarrow \Re_{X}(\mathfrak{S}) \rightarrow \Psi_{0, X}^{*} \rightarrow C_{0, X}\left(S^{*} \Omega, \mathfrak{L}(E)\right) \rightarrow 0 \text { and } 0 \rightarrow \Re_{X}(\mathfrak{E}) \rightarrow \Psi_{X}^{*} \rightarrow \mathfrak{S}_{X} \rightarrow 0 \text {. }
$$

One then defines the $K$-theory $K_{X}\left(T^{*} X \times Y\right)$ as the group of stable homotopy classes of Clifford symbols $\sigma$ satisfying: the map $\operatorname{Pr}_{X}$ : Supp $\sigma \rightarrow X$ is proper (instead of (c) of Definition 1.3).

The map $\Psi^{*}$ is then defined from $K_{X}\left(T^{*} X \times Y\right)$ to $K K(X, Y)$.

(b) The above construction of the map $\Psi^{*}$ works (with obvious minor changes) when one replaces $\mathbb{K}^{0}$ by $K^{1}$ and $K K^{0}$ by $K K^{1}$ (The bundle $E$ of Defi-

(*) Note that $\mathfrak{S}(\Omega, E)$ is $\mathscr{Z} / 2$ graded. Also $\Psi^{*}(\Omega, E)$ is a graded subalgebra of $\mathscr{\Omega}(\mathfrak{S}(\Omega, E)$ ), and the map $\sigma: \Psi *(\Omega, E) \rightarrow \Im(\Omega, E)$ is grading preserving. 
nition 1.3 is not graded here).

(c) The groups $K\left(T^{*} X \times Y\right)$ and $K K(X, Y)$ are independent of the smooth structure of $X$ (using microbundles for the first term-see Appendix B). However the map $\Psi^{*}$ uses the $\mathbb{C}^{\infty}$ structure (in order to construct pseudo-differential operators). It is not clear whether it depends upon the choice of a smooth structure or not (cf. Remark $2.10 \mathrm{c}$ ).

(d) It turns out (Corollary 3.9) that the map $\Psi^{*}$ is an isomorphism.

Let now $X_{1}, X_{2}$ be smooth compact manifolds and $Y$ a locally compact space. Let $\left(\Omega_{1}, E^{1}, \sigma_{1}\right) \in \mathbb{K}\left(T^{*} X_{1} \times X_{2}\right)$ and $\left(\Omega_{2}, E^{2}, \sigma_{2}\right) \in K\left(T^{*} X_{2} \times Y\right)$ be Clifford symbols. We next compute the Kasparov product $\Psi^{*}\left(\sigma_{1}\right) \otimes_{X_{2}} \Psi^{*}\left(\sigma_{2}\right) \in K K\left(X_{1}, Y\right)$.

To do so, we define the cup product of the symbols $\sigma=\sigma_{1} \times_{X_{2}} \sigma_{2} \in K\left(T^{*}\left(X_{1}\right.\right.$ $\left.\left.\times X_{2}\right) \times Y\right)$. We then prove the equality $\Psi^{*}\left(\sigma_{1}\right) \otimes_{C\left(X_{2}\right)} \Psi^{*}\left(\sigma_{2}\right)=\operatorname{Pr}_{X_{1}{ }^{*}}\left(\Psi^{*}(\sigma)\right)$ where $\operatorname{Pr}_{X_{1}}: X_{1} \times X_{2} \rightarrow X_{1}$ is the projection.

Put $X=X_{1} \times X_{2}$, and let $\Omega$ be the open subset of $X \times Y, \Omega=\left\{\left(x_{1}, x_{2}, y\right),\left(x_{1}, x_{2}\right)\right.$ $\left.\in \Omega_{1},\left(x_{2}, y\right) \in \Omega_{2}\right\}$. Let $E$ be the graded bundle over $\Omega, E_{\left(x_{1}, x_{2}, y\right)}=E_{\left(x_{1}, x_{2}\right)}^{1} \hat{\otimes} E_{\left(x_{2}, y\right)}^{2}$.

1.6. Definition. A Clifford symbol $\sigma \in \mathfrak{S}(\Omega, E)$ is called a cup product of $\sigma_{1}$ and $\sigma_{2}$ if, and only if it satisfies:

(a) (Connection) $\sigma\left(x_{1}, 0, x_{2}, \xi_{2}, y\right)=1_{E^{1}} \hat{\otimes} \sigma_{2}\left(x_{2}, \xi_{2}, y\right)$ for $\left(x_{1}, x_{2}\right) \in \operatorname{Supp} \sigma_{1}$.

(b) (Positivity) $\left[\sigma_{1}\left(x_{1}, \xi_{1}, x_{2}\right) \hat{\otimes} 1_{E^{2}}, \sigma\left(x_{1}, \xi_{1}, x_{2}, \xi_{2}, y\right)\right] \geq 0^{(*)}$.

The existence and uniqueness (up to homotopy) of a cup product is an easy special case of the proof of Theorem A.3 of the appendix.

Remark. In fact, it is very easy to give a formula for $\sigma$ :

$$
\sigma\left(x_{1}, \xi_{1}, x_{2}, \xi_{2}, y\right)=M^{1 / 2} \sigma_{1}\left(x_{1}, \xi_{1}, x_{2}\right) \hat{\otimes} 1_{E^{2}}+(1-M)^{1 / 2} 1_{E^{1}} \hat{\otimes} \sigma_{2}\left(x_{2}, \xi_{2}, y\right)
$$

where $M$ is a continuous function on $S^{*} \Omega$ with $0 \leq M \leq 1$, such that:

( $\alpha) \quad M\left(x_{1}, 0, x_{2}, \xi_{2}, y\right)=0$ when $\left(x_{1}, x_{2}\right) \in \operatorname{Supp} \sigma_{1}$

( $\beta) \quad M\left(x_{1}, \xi_{1}, x_{2}, 0, y\right)=1$ when $\left(x_{2}, y\right) \in \operatorname{Supp} \sigma_{2}$

(r) $M$ is independent of $\left(\xi_{1}, \xi_{2}\right)$ for $\left(x_{1}, x_{2}, y\right)$ outside some compact $K \subseteq \Omega$.

Let us now compute the Kasparov product $\Psi^{*}\left(\sigma_{1}\right) \otimes_{X_{2}} \Psi^{*}\left(\sigma_{2}\right)$. The map $f_{1} \hat{\otimes} f_{2} \rightarrow f \quad$ where $\quad f\left(x_{1}, x_{2}, y\right)=f_{1}\left(x_{1}, x_{2}\right) \hat{\otimes} f_{2}\left(x_{2}, y\right)\left(f_{1} \in C_{c}\left(\Omega_{1}, E^{1} \otimes \Omega^{1 / 2}\right), f_{2} \in\right.$ $\left.C_{c}\left(\Omega_{2}, E^{2} \otimes \Omega^{1 / 2}\right)\right)$ identifies $\mathfrak{S}\left(\Omega_{1}, E^{1}\right) \hat{\otimes}_{C\left(X_{2}\right)} \mathfrak{\mathcal { E }}\left(\Omega_{2}, E^{2}\right)$ with $\mathfrak{S}(\Omega, E)$.

Let $P_{2} \in \Psi^{*}\left(\Omega_{2}, E^{2}\right)$ and $P \in \Psi^{*}(\Omega, E)$ be such that $\sigma\left(\mathbb{P}_{2}\right)=\sigma_{2}, \sigma(P)=\sigma$ (where $\sigma$ is a cup product of $\sigma_{1}$ and $\sigma_{2}$ ).

We construci $P_{1}$ somewhat more carefully: Write $\sigma_{1}=\sigma_{1}^{\prime}+\sigma_{1}^{\prime \prime}$ where $\sigma_{1}^{\prime} \in$

(*) This is of course the graded commutator. 
$C_{b}\left(\Omega, \mathscr{L}\left(\mathbb{E}^{1}\right)\right)$ and $\sigma_{1}^{\prime \prime}\left(x_{1}, \xi_{1}, x_{2}\right)=0$ outside Supp $\sigma_{1}$. Let $\Omega_{1}^{\prime} \subseteq \Omega$ be the interior of Supp $\sigma_{1}$. We have $\sigma_{1}^{\prime \prime} \in \mathbb{C}_{0}\left(S^{*} \Omega_{1}^{\prime}, \mathfrak{L}\left(\mathbb{E}^{1}\right)\right)$. Let $\mathbb{P}_{1}^{\prime \prime} \in \Psi_{0}^{*}\left(\Omega_{1}^{\prime}, \mathbb{E}^{1}\right) \subseteq \Psi_{0}^{*}\left(\Omega_{1}, \mathbb{E}^{1}\right)$ be such that $\sigma\left(P_{1}^{\prime \prime}\right)=\sigma_{1}^{\prime \prime}$. Let also $\mathbb{P}_{1}^{\prime} \in \Psi^{*}\left(\Omega_{1}, \mathbb{E}^{1}\right)$ be the operator of multiplication by $\sigma_{1}^{\prime}$. Put $\mathbb{P}_{1}=\mathbb{P}_{1}^{\prime}+\mathbb{P}_{1}^{\prime \prime}$.

Note that $\left(\mathbb{P}_{1}^{2}-1\right) \eta,\left[\mathbb{P}_{1}, f\right] \eta \in \mathfrak{S}\left(\Omega_{1}^{\prime}, E^{1}\right) \subseteq \mathfrak{S}\left(\Omega_{1}, E^{1}\right)$ for all $\eta \in \mathcal{S}\left(\Omega_{1}, E^{1}\right)$ and $f \in C\left(X_{1}\right)$. Hence the support of the Kasparov bimodule $\left(S_{\mathcal{C}}\left(\Omega_{1}, E^{1}\right), P_{1}\right)$ is contained in $\mathfrak{S}_{\mathcal{C}}\left(\Omega_{1}^{\prime}, \mathbb{E}^{1}\right)$.

The next lemma shows that $\mathbb{P}$ is a $\mathbb{P}_{2}$ connection for $\left(\mathfrak{S}_{(}\left(\Omega_{1}, \mathbb{E}^{1}\right), \mathbb{P}_{1}\right)$ (Appendix, Definition A.4 and Remark A.6.4).

1.7. Lemmand Let $\eta_{1}, \eta_{1}^{\prime} \in S_{\mathcal{L}}\left(\Omega_{1}, \mathbb{E}^{1}\right)$ and $Q \in \Psi^{*}(\Omega, E)$. Then $\mathbb{T}_{\eta_{1}}^{*} Q T_{\eta_{1}^{\prime}} \in$ $\Psi^{*}\left(\Omega_{2}, E^{2}\right)$ and $\sigma\left(\mathbb{T}_{\eta_{1}}^{*} Q T_{\eta_{1}^{\prime}}\right)=\tau_{2}$ with

$$
\tau_{2}\left(x_{2}, \xi_{2}, y\right)=\int_{x_{1} \in X_{1}}\left\langle\eta_{1}\left(x_{1}, x_{2}\right), \sigma_{Q}\left(x_{1}, 0, x_{2}, \xi_{2}, y\right) \eta_{1}^{\prime}\left(x_{1}, x_{2}\right)\right\rangle \in \mathcal{L}\left(E_{x_{2}, y}^{2}\right) .
$$

Recall that $T_{\eta_{1}}\left(\eta_{2}\right)=\eta_{1} \hat{\otimes} \eta_{2} \in \mathfrak{S}(\Omega, E)\left(\eta_{2} \in \mathfrak{S}\left(\Omega_{2}, E^{2}\right)\right)$. This means that $\mathbb{T}_{\eta_{1}}\left(\eta_{2}\right)\left(x_{1}, x_{2}, y\right)=\eta_{1}\left(x_{1}, x_{2}\right) \hat{\otimes} \eta_{2}\left(x_{2}, y\right)$ and $T_{\eta_{1}}^{*}(\eta)\left(x_{2}, y\right)=\int_{x_{1} \in X_{1}}\left\langle\eta_{1}\left(x_{1}, x_{2}\right), \eta\left(x_{1}, x_{2}, y\right)\right\rangle$.

Proof. It is of course enough to prove this for $\eta_{1}$ and $\eta_{1}^{\prime}$ with small sup-

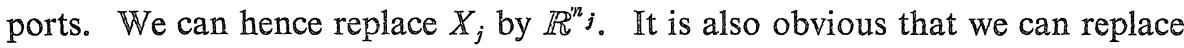
$E^{j}$ by trivial one dimensional bundles. We also assume that $\eta_{1}\left(x_{1}, x_{2}\right)=f_{1}\left(x_{1}\right) f_{2}\left(x_{2}\right)$, $\eta_{1}^{\prime}\left(x_{1}, x_{2}\right)=f_{1}^{\prime}\left(x_{1}\right) f_{1}^{\prime}\left(x_{2}\right)$ where $f_{j}, f_{j}^{\prime} \in \mathbb{C}_{c}^{\infty}\left(\mathbb{R}^{n}{ }^{j}\right)$. By a density argument we may assume that $Q$ is given by the formula

$$
Q h\left(x_{1}, x_{2}, y\right)=\int e^{i\left(\left\langle x_{1}-x_{1}^{\prime}, \xi_{1}\right\rangle+\left\langle x_{2}-x_{2}^{\prime}, \xi_{2}\right\rangle\right)} a\left(x_{1}, \xi_{1}, x_{2}, \xi_{2}, y\right) h\left(x_{1}^{\prime}, x_{2}^{\prime}, y\right) d x_{1}^{\prime} d x_{2}^{\prime} d \xi_{1} d \xi_{2}
$$

where $a$ is a total symbol of order $0\left(h \in C_{c}^{\infty, 0}\left(\mathbb{R}^{n}{ }_{1} \times \mathbb{R}^{n}{ }_{2} \times Y\right)\right)$.

Let $g \in C_{c}^{\infty, 0}\left(R^{n}{ }_{2} \times Y\right)$. Then $\left(\left(T_{\eta_{1}}^{*} Q T_{\eta_{1}^{\prime}}\right) g\right)\left(x_{2}, y\right)$ is given by the formula $\int e^{i\left\langle x_{2}-x_{2}^{\prime}, \xi_{2}\right\rangle} b\left(x_{2}, x_{2}^{\prime}, \xi_{2}, y\right) g\left(x_{2}^{\prime}, y\right) d x_{2}^{\prime} d \xi_{2}$ where

$$
b\left(x_{2}, x_{2}^{\prime}, \xi_{2}, y\right)=\int e^{i\left\langle x_{1}, \xi_{1}\right\rangle} a\left(x_{1}, \xi_{1}, x_{2}, \xi_{2}, y\right) \hat{f}_{1}^{\prime}\left(\xi_{1}\right) f_{2}^{\prime}\left(x_{2}^{\prime}\right) \bar{f}_{1}\left(x_{1}\right) \bar{f}_{2}\left(x_{2}\right) d x_{1} d \xi_{1} .
$$

This $b$ defines a symbol of order 0 (cf. [7], Proposition 4.8) whose principal part is given by:

$$
\begin{aligned}
\tau\left(x_{2}, \xi_{2}, y\right) & =\lim _{t \rightarrow+\infty} b\left(x_{2}, x_{2}, t \xi_{2}, y\right) \\
& =\int e^{i\left\langle x_{1}, \xi_{1}\right\rangle} \sigma_{Q}\left(x_{1}, 0, x_{2}, \xi_{2}, y\right) \hat{f}_{1}^{\prime}\left(\xi_{1}\right) f_{2}^{\prime}\left(x_{2}\right) \bar{f}_{1}\left(x_{1}\right) \bar{f}_{2}\left(x_{2}\right) d x_{1} d \xi_{1} \\
& =\int \sigma_{Q}\left(x_{1}, 0, x_{2}, \xi_{2}, y\right) f_{1}^{\prime}\left(x_{1}\right) f_{2}^{\prime}\left(x_{2}\right) \bar{f}_{1}\left(x_{1}\right) \bar{f}_{2}\left(x_{2}\right) d x_{1}
\end{aligned}
$$


where $\sigma_{Q}\left(x_{1}, \xi_{1}, x_{2}, \xi_{2}, y\right)=\lim _{t \rightarrow+\infty} a\left(x_{1}, t \xi_{1}, x_{2}, t \xi_{2}, y\right)$ is the principal symbol of $Q$.

The difficulty with proving the positivity condition $\left[\mathbb{P}_{1} \hat{\otimes} \mathbb{1}, \mathbb{P}\right] \geq 0$ modulo $\mathfrak{N}\left(\mathfrak{S}(\Omega, E)\right.$ ) (Appendix, Theorem $\left.\mathbb{A}_{0} 3\right)$ comes from the fact that $\mathbb{P}_{1} \hat{\otimes}_{C\left(E_{2}\right)} 1_{\mathfrak{S}\left(\Omega_{2}, E^{2}\right)}$ $\notin \Psi^{*}(\Omega, E)$. Indeed it is of the form (pseudo-differential of order 0 on $\left.X_{1}\right) \hat{\otimes} 1$ which is not pseudo-differential of order 0 (cf. [1], p. 513 or [24], pp. 207 and 210). However, using the next two lemmas we get that $\left[\mathbb{P}_{1} \hat{\otimes} 1, \mathbb{P}\right] \in \Psi^{*}(\Omega, E)$ and has nonnegative symbol.

1.8. Lemma Let $G \in \Psi^{*}(\Omega, E)$ with $\sigma_{G}\left(x_{1}, 0, x_{2}, \xi_{2}, y\right)=0$ for $\left(x_{1}, x_{2}\right) \in$ Supp $\sigma_{1}$. Then $\left(\mathbb{P}_{1} \hat{\otimes} 1\right) G \in \Psi^{*}(\Omega, E)$ and its symbol is given by:

$$
\sigma\left(x_{1}, \xi_{1}, x_{2}, \xi_{2}, y\right)=\left(\sigma_{P_{1}}\left(x_{1}, \xi_{1}, x_{2}\right) \hat{\otimes} 1_{E_{2}}\right) \sigma_{G}\left(x_{1}, \xi_{1}, x_{2}, \xi_{2}, y\right) \text {. }
$$

Note that this symbol makes sense for $\xi_{1} \neq 0$ and extends by continuity to $\xi_{1}=0\left(\xi_{2} \neq 0\right)$.

Proof. Write $P_{1}=\mathbb{P}_{1}^{\prime}+\mathbb{P}_{1}^{\prime \prime}$. Obviously $\mathbb{P}_{1}^{\prime} \hat{\otimes} \in \Psi^{*}(\Omega, E)$. We hence restrict our attention to $P_{1}^{\prime \prime}$. By a density argument we may assume that $\sigma_{G}$ is equal to 0 in some neighborhood of $\left\{\left(x_{1}, x_{2}\right) \in \operatorname{Supp} \sigma_{1}, \xi_{1}=0\right\}$ and hence that there exists $G^{\prime} \in \Psi^{*}(\Omega, E)$ such that $G-\left(\phi+\left(\Delta_{X_{1}} \hat{\otimes} 1\right)\left(\Delta_{X}+1\right)^{-1}\right) G^{\prime} \in \Re(\mathfrak{S}(\Omega, E))$. Here $\phi$ is a multiplication operator by $\phi \in \mathbb{C}_{b}(\Omega), \phi \geq 0$ and $\phi\left(x_{1}, x_{2}, y\right)=0$ if $\left(x_{1}, x_{2}\right)$ $\in \operatorname{Supp} \sigma_{1} ; \Delta_{X_{1}}$ and $\Delta_{X}$ are the Laplacians of $X_{1}$ and $X$.

Now $P_{1}^{\prime \prime} \Delta_{X_{1}}$ is pseudo-differential of order 2. Hence by [1], p. 514 (5.4) (cf. $[24]$, p. 270, Theorem 7) $\left(P_{1}^{\prime \prime} \Delta_{X_{1}}\right) \hat{\otimes} 1$ is in the closure of pseudo-differentials of order 2. So that $\left(\mathbb{P}_{1}^{\prime \prime} \Delta_{X_{1}} \hat{\otimes} 1\right)\left(\Delta_{X}+1\right)^{-1}$ is in the closure of pseudo-differentials of order 0 . As $\left(\mathbb{P}_{1}^{\prime \prime} \hat{\otimes} 1\right) \phi=0$ we get that $\left(\mathbb{P}_{1} \hat{\otimes} 1\right) G \in \Psi^{*}(\Omega, E)$ and has the right symbol.

1.9. Lemma. Let $G \in \Psi^{*}(\Omega, E)$. Assume that $\sigma_{G}=\sigma(G)$ satisfies: $\sigma_{G}\left(x_{1}, \xi_{1}\right.$, $\left.x_{2}, \xi_{2}, y\right) \in \mathbb{1}_{E^{1}} \hat{\otimes} \mathfrak{L}\left(E^{2}\right)$ and $\sigma_{G}\left(x_{1}, 0, x_{2}, \xi_{2}, y\right)$ is independent of $x_{1}$ for $\left(x_{1}, x_{2}\right) \in$ Supp $\sigma_{1}$. Then $\left[P_{1} \hat{\otimes} 1, G\right] \in \Re(\mathfrak{Q}(\Omega, E))$.

Proof. Using Lemma 1.7 we get that $G$ is a $Q_{2}$ connection where $Q_{2} \in$ $\Psi^{*}\left(\Omega_{2}, E^{2}\right)$ satisfies $\mathbb{1}_{E} 1 \hat{\otimes} \sigma\left(Q_{2}\right)\left(x_{2}, \xi_{2}, y\right)=\sigma\left(x_{1}, 0, x_{2}, \xi_{2}, y\right)$ for all $\left(x_{1}, x_{2}\right) \in \operatorname{Supp} \sigma_{1}$. Therefore, for all $k \in \mathfrak{\Omega}\left(\mathfrak{S}\left(\Omega_{1}^{\prime}, E^{\prime}\right)\right.$, we have $[k \hat{\otimes} 1, G] \in \mathfrak{R}(\mathfrak{S}(\Omega, E))$.

Using Lemma 1.8 we get that $\left[\mathbb{P}_{1} \hat{\otimes} \mathbb{1}, G\right]$ only depends, up to $\Omega(S(\Omega, E)$ ), upon the transverse symbol $\sigma_{G}\left(x_{1}, 0, x_{2}, \xi_{2}, y\right)$. Note also that $\left[\mathbb{P}_{1}^{\prime} \hat{\otimes} 1, G\right] \in$ $\Re(\mathfrak{R}(\Omega, \mathbb{E}))$.

Thanks to the above remarks, we know that the problem is local and only depends upon the symbols. We thus may assume that $X_{1}=\mathbb{T}^{n}{ }_{1}$ and that $G$ is 
invariant under translations by $\mathbb{T}^{n}{ }^{n}$ (for a given trivialization of the bundle $E^{1}$ along T $^{n}{ }^{1}$ ).

Let $Q_{1} \in \Psi_{0}^{*}\left(\mathbb{T}^{n}{ }_{1} \times X_{2}, E^{1}\right)$ be of the form $Q_{1}=\phi_{1} Q_{1}^{\prime}$ where $\phi_{1} \in C\left(\mathbb{T}^{{ }_{1}} \times\right.$ $\left.X_{2}, \mathfrak{L}\left(E^{1}\right)\right)$ is a multiplication operator and $Q_{1}^{\prime}$ is $\mathbb{T}^{{ }^{n}{ }^{1}}$ translation invariant. Then $\left[Q_{1}^{\prime} \hat{\otimes} 1, G\right]=0$ and $\left[\phi_{1} \hat{\otimes} 1, G\right] \in \Re(\mathfrak{S}(\Omega, E))$.

The result now follows from the fact that symbols of the form $\sigma\left(Q_{1}\right)$ generate

$$
C\left(S^{*} \mathbb{T}^{n}{ }_{1} \times X_{2}, \mathfrak{Q}\left(E^{1}\right)\right)=\mathfrak{S}\left(X_{1} \times X_{2}, E^{1}\right) \text {. }
$$

1.10. Theorem. Let $\sigma_{1} \in K\left(T^{*} X_{1} \times X_{2}\right), \sigma_{2} \in K\left(T^{*} X_{2} \times Y\right)$ be Clifford symbols. Let $\sigma \in K\left(T^{*}\left(X_{1} \times X_{2}\right) \times Y\right)$ be a cup product of $\sigma_{1}$ and $\sigma_{2}$. Then one has $\Psi^{*}\left(\sigma_{1}\right)$ $\otimes_{X_{2}} \Psi^{*}\left(\sigma_{2}\right)=\operatorname{pr}_{X_{1^{*}}}\left(\Psi^{*}(\sigma)\right)\left(\operatorname{pr}_{X_{1}}: X_{1} \times X_{2} \rightarrow X_{1}\right.$ is the projection).

Proof. Let $P, P_{1}, P_{2}$ be as above. By Lemma 1.7 we know that $P$ is a $P_{2}$ connection for $\left(\mathfrak{S}\left(\Omega_{1}, E^{1}\right), P_{1}\right)$.

Write now $\sigma=\sigma^{\prime}+1_{E_{1}} \hat{\otimes} \sigma^{\prime \prime}$ where $\sigma^{\prime}\left(x_{1}, 0, x_{2}, \xi_{2}, y\right)=0$ for $\left(x_{1}, x_{2}\right) \in \operatorname{Supp} \sigma_{1}$. Let $P=P^{\prime}+P^{\prime \prime}$ be a corresponding decomposition. By Lemma 1.9 we get $\left[P_{1} \hat{\otimes} 1, P^{\prime \prime}\right] \in \Re(\mathfrak{S}(\Omega, E))$. By Lemma $1.8, Q=\left[P_{1} \hat{\otimes} 1, P^{\prime}\right] \in \Psi^{*}(\Omega, E)$ and $\sigma(Q)$ $=\left[\sigma_{1} \hat{\otimes} 1, \sigma^{\prime}\right]=\left[\sigma_{1} \hat{\otimes} 1, \sigma\right] \geq 0$.

1.11. Remarks. (a) When $X_{1}$ and $X_{2}$ are not compact the cup product of the symbols is defined by the same conditions. Note however that $\operatorname{Supp} \sigma_{1}$ is not compact but the projection $\operatorname{Supp} \sigma_{1} \rightarrow X_{1}$ is proper. Also $\operatorname{Supp} \sigma$ is not compact but the projection Supp $\sigma \rightarrow X_{1}$ is proper. Note here that it wouldn't be enough to require that the projection Supp $\sigma \rightarrow X=X_{1} \times X_{2}$ is proper. Indeed such a $\sigma$ would then only give an element $\Psi^{*}[\sigma] \in K K\left(X_{1} \times X_{2}, Y\right)$ which does not project to $K K\left(X_{1}, Y\right)$ when the map $X_{1} \times X_{2} \rightarrow X_{1}$ is not proper.

Let $K_{X_{1}}\left(T^{*}\left(X_{1} \times X_{2}\right) \times Y\right)$ be the $K$-theory of Clifford symbols $\sigma$ whose support satisfies Supp $\sigma \rightarrow X_{1}$ is proper. We get a map $\Psi_{X_{1}}^{*}: K_{X_{1}}\left(T^{*}\left(X_{1} \times X_{2}\right)\right.$ $\times Y) \rightarrow K K\left(X_{1}, Y\right)$ based on an exact sequence $0 \rightarrow \Re_{X_{1}}(\mathcal{S}(\Omega, E)) \rightarrow \Psi_{X_{1}}^{*}(\Omega, E) \rightarrow$ $\mathfrak{S}_{X_{1}}(\Omega, E) \rightarrow 0$ (cf. Remark 1.5 (a)).

The equality of Theorem 1.10 reads: $\Psi_{X_{1}}^{*}\left(\sigma_{1} \times_{X_{2}} \sigma_{2}\right)=\Psi^{*}\left(\sigma_{1}\right) \otimes_{X_{2}} \Psi^{*}\left(\sigma_{2}\right)$. Note that when $X_{2}$ is compact $K_{X_{1}}\left(T^{*}\left(X_{1} \times X_{2}\right) \times Y\right)$ and $K_{X_{1} \times X_{2}}\left(T^{*}\left(X_{1} \times X_{2}\right) \times Y\right)$ coincide. In that case we have $\Psi_{X_{1}}^{*}=\operatorname{pr}_{X_{1} *} \circ \Psi^{*}$.

(b) In all the above construction one may obviously replace $K^{0}$ by $K^{1}$ and $K K^{0}$ by $K K^{1}$.

(c) When $\Omega_{1}=X_{1} \times X_{2}, \Omega_{2}=X_{2} \times Y$ the computation of the Kasparov product simplifies: In that case $P_{\sigma}$ is an $\mathscr{S}\left(\Omega_{1}, E^{1}\right)$ connection (not only an $\left.\left(S_{1}, E^{1}\right), P_{1}\right)$ connection). Also a simple formula for a cup product of the 
symbols is given by:

$$
\begin{aligned}
& \sigma\left(x_{1}, \xi_{1}, x_{2}, \xi_{2}, y\right) \\
& =\left(\left\|\xi_{1}\right\|^{2}+\left\|\xi_{2}\right\|^{2}\right)^{-1 / 2}\left(\left\|\xi_{1}\right\| \sigma_{1}\left(x_{1}, \xi_{1}, y\right) \hat{\otimes} 1_{E^{2}}+1_{E^{1}} \hat{\otimes}\left\|\xi_{2}\right\| \sigma_{2}\left(x_{2}, \xi_{2}, y\right)\right) .
\end{aligned}
$$

However, in all our examples it would be artificial to replace $\Omega$ by $X \times Y$.

(d) For each Kasparov bimodule $(\mathcal{E}, F)$ over $C_{0}\left(X_{1}\right), C\left(X_{2}\right)$ (with $X_{2}$ compact) let $j(\mathcal{E}, F)$ be the Kasparov bimodule $(\mathcal{E}, F)$ over $C_{0}\left(X_{1} \times X_{2}\right), C\left(X_{2}\right)$ where the $C_{0}\left(X_{1}\right) \otimes C\left(X_{2}\right)$ action is given by $\left(f_{1} \otimes f_{2}\right) \xi=f_{1} \xi f_{2}$. Theorem 1.10 can be formulated more precisely as the equality: $\Psi^{*}(\sigma)=j\left(\Psi^{*}\left(\sigma_{1}\right)\right) \otimes_{C\left(X_{2}\right)} \Psi^{*}\left(\sigma_{2}\right)$. (Note that with the notations of [19], $\S 4$, Definition $4, j(\mathcal{E}, F)=\Delta^{*} \tau_{C\left(X_{2}\right)}(\mathcal{E}, F)$ where $\Delta: X_{2} \rightarrow X_{2} \times X_{2}$ is the diagonal map).

(e) One may take $\sigma_{1} \in \mathbb{K}\left(T^{*} X_{1} \times Y_{1} \times Z\right), \sigma_{2} \in K\left(T^{*}\left(Z \times X_{2}\right) \times \mathbb{Y}_{2}\right),\left(X_{j}, Z\right.$ smooth compact manifolds, $Y_{j}$ locally compact spaces). The Kasparov product $\Psi^{*}\left(\sigma_{1}\right) \otimes_{Z} \Psi^{*}\left(\sigma_{2}\right) \in K K\left(X_{1} \times X_{2}, Y_{1} \times Y_{2}\right)$ is then equal to $\left(\mathrm{pr}_{X_{1} \times X_{2}}\right)^{*}\left(\Psi^{*}(\sigma)\right)$ for any Clifford symbol $\sigma \in K\left(T^{*}\left(X_{1} \times Z \times X_{2}\right) \times Y_{1} \times Y_{2}\right)$ satisfying:

(a) (Connection) $\sigma\left(x_{1} .0, z, \eta, x_{2}, \xi_{2}, y_{1}, y_{2}\right)=1_{E^{1}} \hat{\otimes} \sigma_{2}\left(z, \eta, x_{2}, \xi_{2}, y_{2}\right)$ for $\left(x_{1}, y_{1}, z\right) \in \operatorname{Supp} \sigma_{1}$.

(b) (Positivity) $\left[\sigma_{1} \hat{\otimes} \mathbb{1}_{E^{1}}, \sigma\right] \geq 0$ 。

\section{§IH.Wrong Way Fuanctori̊linity}

In this section we prove the formula $(g \circ f) !=f ! \otimes g !$ stated as a problem in [10], $\S 10$ 。

Let us first recall the construction of $f !$ (Cf. [10], p. 599):

Let $X$ and $Y$ be smooth manifolds and $f: X \rightarrow Y$ a continuous map. We assume that $f$ is $K$-oriented; this means that the Euclidean vector bundle $\mathbb{T}_{f}=$ $T^{*} X \oplus f^{*} T Y$ is endowed with a $\operatorname{Spin}^{c}$ structure $S$. Here $S$ is a complex Hermitian vector bundle; each $\xi \in\left(T_{f}\right)_{x}$ defines an endomorphism $c(\xi)$ of the fiber $S_{z}$ $(x \in X)$ such that:

(a) The map $\xi \rightarrow c(\xi)$ is linear.

(b) $c(\xi)=c(\xi)^{*}, c(\xi)^{2}=\|\xi\|^{2}$ (Clifford condition).

(c) $S_{x}$ is irreducible as a module over $\operatorname{Cliff}_{C}\left(T_{f_{x}}\right)$ (i.e. $\operatorname{dim} S_{x}=2^{m}$ where $m$ is the integral part of $\frac{\operatorname{dim} X+\operatorname{dim} Y}{2}$ ).

If moreover $\operatorname{dim} X+\operatorname{dim} Y$ is even:

$(d)^{(*)} \quad$ The bundle $S$ is $\mathbb{Z} / 2$ graded and $c(\xi)$ is of degree 1 for all $\xi$.

Let us assume that the Riemannian metric on $Y$ is chosen with injectivity

(*) Or equivalently $T_{f}$ is oriented. This is automatic by (c) in the odd dimensional case. 
radius 1 . For $y_{1}, y_{2} \in Y$ with $d\left(y_{1}, y_{2}\right)<1$, we let $y_{2}-y_{1} \in T_{y_{1}}(Y)$ be the unique tangent vector of norm less than 1 , whose exponential is equal to $y_{2}\left(\exp _{y_{1}}\left(y_{2}-y_{1}\right)\right.$ $\left.=y_{2}\right)$. Let $\Omega$ be the open subset of $X \times Y, \Omega=\{(x, y), d(f(x), y)<1\}$.

Put $\left.\sigma(x, \xi, y)=M^{1 / 2}(x, y)\|\xi\|^{-1} c(\xi, 0)+(1-M)^{1 / 2}(x, y) \| y-f(x)\right) \|^{-1} c(0, y-$ $f(x)) \in \mathfrak{R}\left(S_{x}\right)\left((x, y) \in \Omega, \xi \in T_{x}^{*} X, \xi \neq 0\right)$ where $M(x, y)=1$ if $d(x, y)<\varepsilon$ and $M(x, y)=0$ if $d(x, y)>1-\varepsilon(\varepsilon>0)$.

Then $\left(\Omega, \operatorname{pr}_{x}^{*} S, \sigma\right)$ defines a Clifford symbol (Definition 1.3). Let $d$ be equal modulo 2 to $\operatorname{dim} X+\operatorname{dim} Y$.

2.1. Definition ([10], $\S 10$, p. 599). Let $f: X \rightarrow Y$ be a $K$ oriented map. The element $f ! \in K K^{d}(X, Y)$ is defined to be $\Psi^{*}([\sigma])$. Here $[\sigma]$ denotes the class of the Clifford symbol $\sigma$ in $K_{X}^{d}\left(T^{*} X \times Y\right)$ (cf. Remark 1.5 (a)).

Let us first check that this construction of elements of $K K(X, Y)$ is not trivial, proving that $\left(\operatorname{Id}_{X}\right)$ ! is equal to the unit $1_{X}$ of the $\operatorname{ring} K K(X, X)$.

Note that $K(X)$ is a subring of $K K(X, X)$ : to each Hermitian vector bundle $E$ one associates the $C(X), C(X)$ bimodule $C(X ; E)$. As $C(X)$ acts on the left by compact operators this defines an element $(E)=j([E])$ of $K K(X, X)$ (see Remark $1.11(d))$.

Let $\mathcal{E}$ be a $C_{0}(X), C_{0}(Y)$ bimodule. We say that the support of $\mathcal{E}$ is disjoint from $\Omega=\Omega_{1} \times \Omega_{2} \subset X \times Y$ if and only if for all $f_{1} \in C_{c}\left(\Omega_{1}\right) \subset C_{0}(X), f_{2} \in C_{c}\left(\Omega_{2}\right)$ and $\xi \in \mathcal{E}$ we have $f_{1} \xi f_{2}=0$. This defines the support of $\mathcal{E}$ as a closed subset of $X \times Y$.

2.2. Lemma. Let $X$ be a smooth compact manifold. Let $p$ be the map from $X$ to $\mathrm{pt}$ and $p_{*}: K K(X, X)=K K(\mathrm{pt}, X)=K(X)$ the corresponding map. Then $p_{*} \circ j$ $=\mathrm{id}_{K(X)}$. Moreover, there exists a neighbourhood $\Omega$ of $\Delta(X)=\{(x, x), x \in X\}$ such that for every Kasparov $C(X), C(X)$ bimodule $(\mathcal{E}, F)$ with $\operatorname{Supp} \mathcal{E} \subseteq \Omega$ one has: $j \circ p_{*}[(\mathcal{E}, F)]=[(\mathcal{E}, F)]$.

Proof. Choose a Riemannian metric in $X$ with injectivity radius 1 and put $\Omega=\{(x, y), d(x, y)<1\}$. Let $\mathcal{E}$ be a $C(X), C(X)$ bimodule whose support is in \&. Consider $\mathcal{E}$ as the space of continuous sections of the continuous field of Hilbert spaces $\left(\mathcal{E}_{x}\right)_{x \in X}$. Let then $\left(\phi_{t}\right)_{t \in[0,1]}$ be the family of $C(X)$ actions on $\mathcal{E}$ given by $\phi_{t}(f) \xi=\left(f \circ p_{t, x}\right) \xi$ where $p_{t, x}(y)=\exp _{x} t(y-x)\left(\xi \in \mathcal{E}_{x},(x, y) \in \Omega\right)$.

Using $\phi_{t}$ we get a homotopy between $(\mathcal{E}, F)$ and $\left(\mathcal{E}_{1}, F\right)$ where $\mathcal{E}_{1}$ is a commutative bimodule (i.e. satisfies $f \xi=\xi f$ for all $f \in C(X) \xi \in \mathcal{E}_{1}$ ). But then by definition of $p_{*}$ and $j$ one has $\left[\left(\varepsilon_{1}, F\right)\right]=j \circ p_{*}\left[\left(\varepsilon_{1}, F\right)\right]$.

2.3. Remarks. (a) If $X$ is not compact, the $\operatorname{ring} \operatorname{Vect}(X)$ of stable isomor- 
phism classes of complex vector bundles is equal to the subring of $K K(X, X)$ of classes of commutative bimodules. The above proof shows that if $(\mathcal{E}, F)$ is a Kasparov bimodule such that the support of $\mathcal{E}$ is contained in a suitable neighbourhood of the diagonal, its class $[(\varepsilon, F)]$ belongs to Vect $X$.

(b) Let $(\mathcal{E}, F)$ be a Kasparov $C(X), C(X)$ bimodule and let $\mathcal{E}^{\prime}$ be its support (Definition A.4).

If the support of the $C(X), C(X)$ bimodule $\mathcal{E}^{\prime}$ is contained in the neighbourhood $\Omega$ of $\Delta(X)$ (of Lemma 2.2) then $j \circ p_{*}[(\mathcal{E}, F)]=[(\mathcal{E}, F)]$ (see Remark A.6.2).

(c) In the above lemma $X$ does not need to be a smooth manifold. If $X$ is just a topological manifold there still exists (by [21], Theorem 2) a neighbourhood $\Omega$ of the diagonal $\Delta$ in $X \times X$ such that the first projection $\operatorname{pr}_{1}: \Omega \rightarrow X$ is a retraction by deformation of $\Omega$ to $\Delta$. Hence the proof of Lemma 2.2 applies.

Let now $E$ be a Euclidean vector bundle over a locally compact space $X$. Consider the continuous family of Hilbert spaces $\left(\mathfrak{S}_{x}\right)_{x \in X}, \mathfrak{E}_{x}=\mathbb{L}^{2}\left(\mathbb{E}_{x} ; A_{C} \mathbb{E}_{x}\right)$, graded by even and odd forms.

Let $M$ be a continuous function on $\mathbb{R}^{+}, 0 \leq M \leq 1$, which is 0 around 0 and 1 around $+\infty$. For $x \in X, \xi \in E_{x}, \eta \in E_{x}^{*} \simeq E_{x}(\eta \neq 0)$ let $\alpha_{x}(\xi, \eta) \in \mathbb{R}\left(\Lambda_{C} E_{x}\right)$ be defined by $\alpha_{x}(\xi, \eta)(\omega)=\left(M^{1 / 2}(\| \xi||)\|\xi\|^{-1} \xi+i(1-M)^{1 / 2}(\|\xi\|)\|\eta\|^{-1} \eta\right) \wedge \omega$.

Let $\left(P_{x}\right)_{x \in X}$ be a continuous family of order zero pseudo-differential operators trivial at $\infty\left(P_{x}\right.$ acting on $\left.\mathfrak{S}_{x}\right)$ whose principal symbol is given by $\sigma_{x}(\xi, \eta)=$ $\alpha_{x}(\xi, \eta)+\alpha_{x}(\xi, \eta)^{*}$.

The pair $(\mathfrak{S}, P)$ defines an element of $K K(X, X)$ (see Section $\mathbb{I}$ ). We have:

2.4. Lemma. (cf. [16]) The commutative bimodule $\mathfrak{S}$ with the endomorphism $P$ defines the unit element $1_{X} \in K K(X, X)$.

Proof. Assume for simplicity that $X$ is compact. As the bimodule $\mathfrak{S}$ is commutative we have $[(\mathfrak{S}, P)]=j \circ p_{*}[(\mathfrak{S}, P)]$. Now the class of $p_{*}[(\mathfrak{S}, P)]$ in $K(X)$ is the index of the family $\left(P_{x}^{+}\right)_{x \in X}$ where $P_{x}^{+}$is the restriction of $P_{x}$ to $\mathfrak{S}_{x}^{(0)}$ $\left(P_{x}^{+}: \mathfrak{S}_{x}^{(0)} \rightarrow \mathfrak{S}_{x}^{(1)}, \mathfrak{S}_{x}^{(0)}\right.$ and $\mathfrak{S}_{x}^{(1)}$ are the homogeneous parts of the graded Hilbert space $\mathfrak{S}_{x}$ ). Now by [16], Theorem 5.3 the equivariant $O(n)$ index of $F_{x}^{+}$is 1 . Hence, the index of the family $\left(P_{x}^{+}\right)_{x \in X}$ is the trivial one dimensional bundle.

Let $X$ be a smooth manifold. Recall that the bundle $\mathbb{T}_{\operatorname{Ia}_{X}}=T^{*} X \oplus T X$ admits the following $\operatorname{Spin}^{c}$ structure: $S_{x}=\Lambda_{C} T_{x} X$, and for $\xi \in T_{x}^{*} X, \nu \in \mathbb{T}_{x} X$ $c(\xi, \nu)=\alpha(\xi, \nu)+\alpha(\xi, \nu)^{*}$, where $\alpha(\xi, \nu)(\omega)=(i \xi+\nu) \wedge \omega,\left(\omega \in \Lambda_{C} T_{x} X\right)$.

2.5. Proposition. Let $X$ be a smooth manifold and let $\mathbb{I d}_{X}$ be $\mathbb{K}$-oriented as shown above. Then $\left(\mathrm{Id}_{X}\right)$ ! is the unit element $1_{X}$ of the ring $K K(X, X)$. 
Proof. Let $\Omega$ be a neighbourhood of the diagonal $\Delta$ in $X \times X$ and $\Psi: T X \rightarrow$ $\Omega$ a diffeomorphism of the form $\Psi(x, \nu)=\left(x, \exp _{x}(1+\|\nu\|)^{-1} \nu\right)^{(*)}$. Let $(\mathfrak{C}, P)$ be the Kasparov bimodule corresponding to the tangent bundle $T X$ as in Lemma 2.4. If we transport $(\mathfrak{C}, P$ ) to $\Omega$ through the diffeomorphism $\Psi$, we get $j \circ$ $p_{*}\left(\operatorname{Id}_{X}\right)$ !. Now by Lemma $2.2 j \circ p_{*}\left(\operatorname{Id}_{X}\right) !=\left(\operatorname{Id}_{X}\right)$ ! and by Lemma $2.4(\mathfrak{S}, P)=$ $1_{X}$. Hence $\left(\mathbb{I d}_{X}\right) !=1_{X}$.

Let now $X_{1}, X_{2}$ and $Y$ be smooth manifolds and let $f_{1}: X_{1} \rightarrow X_{2}$ and $f_{2}$ : $X_{2} \rightarrow Y$ be $K$-oriented continuous maps. Let $S_{1}$ and $S_{2}$ be the corresponding Spin ${ }^{c}$ structures of the bundles $T_{f_{1}}$ and $T_{f_{2}}$. Let also $S_{X_{2}}$ be the $\operatorname{Spin}^{c}$ structure of the bundle $T_{\operatorname{Id}_{X_{2}}}$ which is discussed above. Then there exists a unique $\operatorname{Spin}^{c}$ structure $S$ of $T_{f_{2}^{\circ} f_{1}}$ such that the Spin structures $S_{1} \hat{\otimes} f_{2}^{*} S_{2}$ and $f_{2}^{*} S_{X_{2}} \hat{\otimes} S$ of $T_{f_{1}} \oplus f_{2}^{*} T_{f_{2}} \simeq f_{2}^{*} T_{\operatorname{Id~}_{X_{2}}} \oplus T_{f_{2^{\circ} f_{1}}}$ coincide (see Appendix B, Proposition B.6.(c)).

2.6. Theorem. Let $X_{1}, X_{2}, Y$ be smooth manifolds, $f_{1}: X_{1} \rightarrow X_{2}, f_{2}: X_{2} \rightarrow Y$ be $K$-oriented continuous maps. Let $f_{2} \circ f_{1}$ be K-oriented as shown above. We then have $\left(f_{2} \circ f_{1}\right) !=f_{1} ! \otimes_{X_{2}} f_{2} !$.

Proof. Assume for simplicity that $X_{1}$ and $X_{2}$ are compact. We shall compare two elements of the form $\Psi^{*}[\sigma]$ of $K K\left(X_{1} \times X_{2}, Y\right)$.

The first one is $j\left(f_{1} !\right) \otimes_{X_{2}} f_{2}$ ! and hence (Theorem 1.10, Remark $1.11(\mathrm{~d})$ ) is of the form $\Psi^{*}[\sigma]$ where $\sigma \in K\left(T^{*}\left(X_{1} \times X_{2}\right) \times Y\right)$ is a cup product of $\sigma_{1}$ and $\sigma_{2}$ $\left(\sigma_{1} \in K\left(T^{*} X_{1} \times X_{2}\right)\right.$ and $\sigma_{2} \in K\left(T^{*} X_{2} \times Y\right)$ are the Clifford symbols corresponding to the $K$-orientations of $f_{1}$ and $f_{2}$ - see Definition 2.1).

Let us define the second. Let $\delta_{2} \in K\left(T^{*} X_{2} \times X_{2}\right)$ be the Clifford symbol corresponding to the $K$-orientation of $\operatorname{Id}_{X_{2}}$. Let $\sigma_{1}^{\prime} \in K\left(T^{*} X_{2} \times X_{1}\right)$ be equal to $\left(\operatorname{id}_{T^{*} X_{2}} \times f_{1}\right)^{*}\left(\delta_{2}\right)$. Let now $u \in K K\left(X_{1} \times X_{2}, X_{1}\right)$ be equal to $j\left(\Psi^{*}\left[\sigma_{1}^{\prime}\right]\right)$. As we have $\Psi^{*}\left(\left[\sigma_{1}^{\prime}\right]\right)=f_{1}^{*} \Psi^{*}\left(\delta_{2}\right)=f_{1}^{*}\left(1_{X_{2}}\right)$ (Proposition 2.5), $u$ is equal to $g_{*}\left(1_{X_{1}}\right)$, where $g$ : $X_{1} \rightarrow X_{1} \times X_{2}$ is defined by $g\left(x_{1}\right)=\left(x_{1}, f_{1}\left(x_{1}\right)\right)$.

Let $\sigma_{2}^{\prime}$ be the symbol of $\left(f_{2} \circ f_{1}\right)$ !. Let $\sigma^{\prime} \in K\left(T^{*}\left(X_{1} \times X_{2}\right) \times Y\right)$ be a cup product of $\sigma_{1}^{\prime}$ and $\sigma_{2}^{\prime}$. Then $u \otimes_{X_{1}}\left(f_{2} \circ f_{1}\right) !=\Psi^{*}\left[\sigma^{\prime}\right]$.

By the choice of the $K$-orientation of $f_{2} \circ f_{1}$ we have $[\sigma]=\left[\sigma^{\prime}\right]$ in $K\left(T^{*}\left(X_{1} \times\right.\right.$ $\left.X_{2}\right) \times Y$ ).

We thus get the equality $j\left(f_{1} !\right) \otimes_{X_{2}} f_{2} !=g_{*}\left(f_{2} \circ f_{1}\right)$ !. Let $p: X_{1} \times X_{2} \rightarrow X_{1}$ be the projection. We have $p_{*}\left(j\left(f_{1} !\right) \otimes_{X_{2}} f_{2} !\right)=f_{1} ! \otimes_{X_{2}} f_{2} !$ and $p \circ g=\mathbb{I d}_{X_{1}}$. Hence $\left(f_{2} \circ f_{1}\right) !=f_{1} ! \otimes_{X_{2}} f_{2} !$

Let us state an easy corollary of this theorem. Note that if $f_{1}$ and $f_{2}$ :

(*) $X$ is endowed with a Riemannian structure with injectivity radius equal to 1 . 
$X \rightarrow Y$ are homotopic and $f_{1}$ is $K$-oriented, then $f_{2}$ is naturally $K$-oriented and one has $f_{1} !=f_{2}$ ! (even if $f_{1}$ and $f_{2}$ are proper, we do not have to assume that the homotopy is through proper maps).

2.7. Corollary. Let $X$ and $Y$ be smooth manifolds and $f: X \rightarrow Y$ a K-oriented homotopy equivalence. Then $f$ ! defines a natural isomorphism of $K^{*}(A, B \otimes$ $\left.C_{0}(X)\right)$ with $K K^{*}\left(A, B \otimes C_{0}(Y)\right)$ and of $K K^{*}\left(A \otimes C_{0}(X), B\right)$ with $K K^{*}\left(A \otimes C_{0}(Y)\right.$, $B)^{(*)}$.

Proof. Let $g: Y \rightarrow X$ be a homotopy inverse of $f$. As $f \circ g$ and $f$ are $K$ oriented so is $g$ (see Appendix B, Proposition 6.c) and we have $g ! \otimes_{X} f !=(f \circ g)$ ! $=1_{Y}$ and $f ! \otimes_{Y} g !=(g \circ f) !=1_{X} . \quad$ The result then follows from [19], Theorem 6 , $\S 4$.

In particular, if $X$ is a contractible smooth manifold then $K K^{*}\left(A \otimes C_{0}(X)\right.$, $B)=K K^{*}\left(A, B \otimes C_{0}(X)\right)=K K^{*}(A, B)$.

We shall now see how $f ! \in K K(X, Y)$ simplifies for immersions and submersions (cf. [10], pp. 598-599).

Let $f: X \rightarrow Y$ be an immersion, $N$ its normal bundle. Let $\tilde{f}$ be the natural extension of $f$ to an etale map from $N$ to $Y(\tilde{f}$ is a diffeomorphism from $N$ to a tubular neighbourhood of $f(X)$ when $f$ is a proper imbedding. In general $\tilde{f}$ is constructed locally using the tubular neighbourhood construction).

As $T_{f}$ is isomorphic to $T_{\operatorname{Id}_{X}} \oplus N, K$-orientations of $f$ and Spin $^{c}$ structures of $N$ are in a one to one correspondence. Assume therefore that $S$ is a $\operatorname{Spin}^{c}$ structure for $N$. Let us define the corresponding element $f_{\mathrm{im}}$ ! of $K K(X, Y)$.

On $C_{c}(N, S)$ consider the $C_{0}(Y)$ valued inner product given by: $\langle\xi, \eta\rangle(y)$ $=\sum_{\tilde{f}(\nu)=y}\langle\xi(\nu), \eta(\nu)\rangle$; the right action of $C_{0}(Y)$ and the left action of $C_{0}(X)$ are given by $(g \xi h)(\nu)=g(\pi(\nu)) \xi(\nu) h(\tilde{f}(\nu))(\pi: N \rightarrow X$ is the projection).

Let $\mathcal{E}$ be the Hilbert $C_{0}(Y)$-module, completion of $C_{c}(N, S)$ with respect to this inner product. Let $F \in \mathfrak{L}(\mathcal{E})$ be given by $(F \xi)(\nu)=(1-M)^{1 / 2}\|\nu\|^{-1} c(\nu) \xi(\nu)$, where $M(\nu)=1$ for $\nu=0$ and $M(\nu)=0$ for large $\|\nu\|$. For $g \in C_{0}(X)$ we have $g F=F g$. Moreover if $g \in C_{c}(X),\left(F^{2}-1\right) g$ has compact support in $N$ so that $\left(F^{2}-1\right) g \in \Re(\mathcal{E})$. Thus the pair $(\mathcal{E}, F)$ defines an element $f_{\text {im }} ! \in K K^{\operatorname{dim} X+\operatorname{dim} Y}(X, Y)$.

2.8. Proposition. Let $f: X \rightarrow Y$ be a K-oriented immersion. Then $f !=f_{\mathrm{im}} !$

Proof. We shall check that $f$ ! as defined in Definition 2.1 is equal to the Kasparov product $\left(\operatorname{Id}_{X}\right) ! \otimes_{X} f_{\text {im }} !$.

(*) Of degree $\operatorname{deg}(f !)=\operatorname{dim} X+\operatorname{dim} Y$. 
Let $S_{1}, S_{2}, S$ be the Spin ${ }^{c}$ structures corresponding to $T_{\operatorname{Id}_{X}}, N$, and $T_{f^{\circ}}$ We have $S=S_{1} \hat{\otimes} S_{2}$. Let $\Omega_{1} \subset X \times X$ be the open neighbourhood of the diagonal $\Delta$ on which $\left(\mathbb{I d}_{X}\right)$ ! is defined. Let $\Omega \subseteq X \times Y$ be the open set defined by $\Omega=\{(x$, $\left.\tilde{f}(\nu)), x \in X, \nu \in N,(x, \pi(\nu)) \in \Omega_{1}\right\}$. Then $\left(\mathbb{I d}_{X_{1}}\right)$ ! is represented by $\left(S_{\mathcal{C}}\left(\Omega_{1}, S_{1}\right), P_{1}\right)$ and $f$ ! is represented by $(\mathfrak{S}(\Omega, S), \mathbb{P})$ (with the notations of Section 1 ). Let $(\mathcal{E}, F)$ represent $f_{\mathrm{im}}$ ! as defined above.

The bimodule $\mathscr{S}_{(}\left(\Omega_{1}, S_{1}\right) \hat{\otimes}_{C_{0}(X)} \mathcal{E}$ identifies easily with $\mathscr{C}(\Omega, S)$.

As $F$ commutes with the action of $C_{0}(X), 1 \hat{\otimes} F \in \mathfrak{L}(\mathfrak{\mathcal { S }}(\Omega, S))$ is well defined and is an $F$-connection (cf. Remark A.6.3). As $\sigma(P)$ agrees with $1 \hat{\otimes} F$ at infinity in $N, P-1 \hat{\otimes} F$ is a 0 connection. Hence $P$ is an $F$ connection.

The positivity condition $\left[P, P_{1} \hat{\otimes} 1\right] \geq 0$ is here easy to check as $P_{1} \hat{\otimes} 1$ is a pseudo-differential family (though not trivial at $\infty$ ).

Let now $f: X \rightarrow Y$ be a smooth submersion. Let $S$ be a $\operatorname{Spin}^{c}$ structure on the bundle $F=\operatorname{Ker}(d f)(\subseteq T X)$.

On $C_{c}\left(X, S \otimes \Omega^{1 / 2}\right)^{(*)}$ consider the $C_{0}(Y)$ valued inner product given by $\langle\xi, \eta\rangle(y)=\int_{f(x)=y}\langle\xi(x), \eta(x)\rangle$; let also $C_{0}(X)$ act on the right and $C_{0}(Y)$ on the left by $(g \xi h)(x)=g(x) \xi(x) h(f(x))$. Let $\varepsilon$ be the $C_{0}(X), C_{0}(Y)$ bimodule completion of $C_{c}\left(X, S \otimes \Omega^{1 / 2}\right)$ with respect to the $C_{0}(Y)$ valued inner product. Let $\left(P_{y}\right)_{y \in Y}$ be a continuous family of order zero pseudo-differential operators acting on $L^{2}\left(f^{-1}\{y\}, S\right)$ with principal symbol $\sigma(x, \xi)=\|\xi\|^{-1} c(\xi)\left(x \in f^{-1}\{y\}, \xi \in T_{x}^{*}\left(f^{-1}\right.\right.$ $\left.\{y\})=\operatorname{ker}\left(d f_{x}\right)^{*}\right)$. To construct $P$ (as an element of $\mathfrak{L}(\mathcal{E})$ ) and check that, for $g \in C_{c}(X), g\left(P^{2}-1\right)$ and $[g, P]$ belong to $\Omega(\mathcal{E})$, one may proceed locally in $X$, and hence assume $X=X_{1} \times Y$ and $f=\operatorname{pr}_{Y}$ where Proposition 1.2 applies.

The pair $(\mathcal{E}, P)$ defines an element $f_{\text {sub }}$ ! of $K K^{*}(X, Y)$.

2.9. Proposition. Let $X$ and $Y$ be smooth manifolds and $f: X \rightarrow Y$ a Koriented submersion. Then $f !=f_{\text {sub }}$ !.

Proof. One checks that $f_{\text {sub }} ! \otimes_{Y} I_{Y} !=f !$. It is clear that the two bimodules coincide. Write $f !=[(\mathcal{E}, P)]$ (as in Definition 2.1). To show that $P$ is a Kasparov product is a local problem on $X$, so that the proof of Theorem 1.10 applies.

2.10. Remarks. (a) Let $f: X \rightarrow Y$ be a continuous oriented map ( $X$ and $Y$ are smooth manifolds). One can define, using Poincaré duality, the push forward $f$ ! in cohomology with compact support, $f$ !: $H_{c}^{*}(X) \rightarrow H_{c}^{*}(Y)$.

If $f$ is $K$-oriented, then it is oriented, but the diagram

(*) Cf. Remark 1.1.1. 


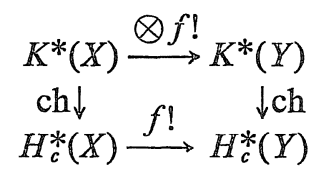

does not commute. But introducing the Todd genus $\operatorname{Td}(f)=\operatorname{Td}(T X) / \operatorname{Td}\left(f^{*} T Y\right)$ $\stackrel{\text { def }}{=} \operatorname{Td}\left(T_{C} X\right) / \operatorname{Td}\left(T_{f}\right) \in H^{*}(X)$, we get $\operatorname{ch}\left(x \otimes_{X} f !\right)=f !(\operatorname{Td}(f) \cdot \operatorname{ch}(x)), x \in K^{*}(X)$. (This is seen by factorizing $f$ through $Y \times \mathbb{R}^{n}$, using an imbedding of $X$ in $\mathbb{R}^{n}$.)

(b) Let $f_{i}: X_{i} \rightarrow Y_{i}(i=1,2)$ be $K$-oriented maps. Then $f_{1} \times f_{2}: X_{1} \times X_{2} \rightarrow$ $Y_{1} \times Y_{2}$ is naturally $K$-oriented. One has rather obviously (using Remark 1.11(e)), $\left(f_{1} \times f_{2}\right) !=f_{1} ! \otimes_{C} f_{2} !$. Combining this with Proposition 2.5 and Theorem 2.6 we get:

Let $f_{1}: X_{1} \rightarrow Y_{1} \times Z$ and $f_{2}: Z \times X_{2} \rightarrow Y_{2}$ be $K$-oriented maps. Let $g: X_{1} \times X_{2}$ $\rightarrow Y_{1} \times Y_{2}$ be the composition $\left(\operatorname{Id}_{Y_{1}} \times f_{2}\right) \circ\left(f_{1} \times \operatorname{Id}_{X_{2}}\right)$. We have $g !=f_{1} ! \otimes_{Z} f_{2}$ ! (cf. [19], Theorem 4, §4).

(c) Let $f: X \rightarrow Y$ be a $K$-oriented map. The element $f$ ! is better described using the $K$-orientation of the microbundle $\tau_{f}=\left|T_{f}\right|=T^{*} X \times_{X} f^{*} \tau_{y}$ (cf. Appen$\operatorname{dix} \mathrm{B}):$ This $K$-orientation is given by a triple $\left(\Omega_{0}, E, \sigma_{0}\right)$, where $\Omega_{0} \subset T^{*} X \times Y$ is a neighbourhood of $\{(x, 0, f(x)), x \in X\}, E$ is a (graded) vector bundle over $X$ and $\sigma_{0}(x, \xi, y) \in \mathfrak{R}(E)$ for $(x, \xi, y) \in \Omega_{0}$ (cf. Definition B. 1).

Let $\Omega \subseteq X \times Y$ be a neighbourhood of the graph of $f$ and $\varepsilon>0$ such that for $(x, y) \in \Omega$ and $\|\xi\| \leq \varepsilon,(x, \xi, y) \in \Omega_{0}$. Let $M \in C_{c}(\Omega)$ be such that $0 \leq M \leq \varepsilon$ and $M(x, f(x))=\varepsilon$ for all $x$ in $X$. For $(x, y)$ in $\Omega$ and $\xi \in T_{x}^{*} X, \xi \neq 0$ put $\sigma(x, \xi, y)$ $=\sigma_{0}\left(x,\|\xi\|^{-1} M(x, y) \xi, y\right)$. Then $(\Omega, \mathbb{E}, \sigma)$ is a Clifford symbol (Definition 1.3). One then defines $f$ ! putting $f !=\Psi^{*}([\sigma])$. The advantage of this presentation is to make it clear that $f$ ! can as well be defined if we just assume that $Y$ is a topological manifold and $f: X \rightarrow Y$ is $K$-oriented.

Obviously Theorem 2.6 remains true if $Y$ is a topological manifold.

Note that at the formal level the map $\sigma_{0} \rightarrow f$ ! is given by the composition $K\left(\tau_{f}\right) \stackrel{e}{\rightarrow} K\left(T^{*} X \times Y\right) \stackrel{\Psi^{*}}{\rightarrow} K K(X, Y)$. Here $e$ is the map $\sigma_{0} \rightarrow \sigma$; it is an "excision type" map $\left(\sigma_{0}\right.$ is an element of $K\left(\Omega_{0}\right)$ and $\Omega_{0}$ is an open subset of $T^{*} X \times Y$ ).

With this presentation, it is obvious that if we change $\sigma_{0}$ to some $u \sigma_{0}$ (where $u$ is a unit of $\left.K^{0}(X)\right), f !$ is changed into $j(u) \otimes_{X} f$ !.

Notice moreover that a $K$-orientation of $f$ is a $K$-orientation of the microbundle $\tau_{X} \times_{X} f^{*} \tau_{Y}$. Hence it makes sense to change the smooth structure of $X$ but keep the same $K$-orientation. We obtain an element $f^{\prime} ! \in K K(X, Y)$. Let us compare $f$ ! and $f^{\prime}$ !.

Call $\delta$ and $\delta^{\prime}$ the $K$-orientations of $K\left(\tau_{X} \times_{X} \tau_{X}\right)$ corresponding to the Spin ${ }^{c}$ 
structures of the complex bundles $T^{*} X \oplus T X$ for the two smooth structures of $X$ (cf. Remark 8.4). Let $u$ be the unit of $K^{0}(X)$ such that $\delta^{\prime}=u \delta$.

Let $g: X \rightarrow X$ be $K$-oriented by $\delta^{\prime}$. We have $g^{\prime} !=1_{X}$ and $g !=j(u)$. But by Theorem 2.6 we have $f !=g ! \otimes_{X} f^{\prime} !$. Hence $f !=j(u) \otimes_{X} f^{\prime} !$. Note that $(u-1)$ is a 2-torsion element of $K^{0}(X)$ (cf. [25]).

\section{$\S$ HH. Composition of Correspondences and Applications}

In this section we shall adopt the point of view according to which the elements of $\operatorname{KK}(A, B)$ are the natural formalisation of the algebraic notion of correspondence. At the topological level, we shall use the definition of correspondence between $X$ and $Y$ of [4] (both $X$ and $Y$ are locally compact spaces with $Y$ a manifold) as given by $\left(Z, E, f_{X}, g_{Y}\right)$ where $Z$ is a smooth manifold, $E$ is a complex vector bundle over $Z, f_{X}: Z \rightarrow X$ is continuous and proper, while $g_{Y}$ : $Z \rightarrow Y$ is continuous and $K$-oriented.

The results of the last section allow to define, for each correspondence, $X \stackrel{f_{X}}{\leftarrow}(Z, E) \stackrel{g_{Y}}{\rightarrow} Y$ an element: $\left(f_{X}\right)_{*}\left((E) \otimes_{Z} g_{Y} !\right) \in K K(X, Y)$ where $(E)=j[E] \in$ $K K(Z, Z)$ is the class of $E$, and plays a rather trivial role as a multiplicity. The main interest of this construction of elements of $K K(X, Y)$ (beyond its surjectivity) is that the Kasparov product has a simple topological interpretation as the composition of correspondences: Take $X, M, Y$ where $X$ is locally compact, $M$ is a smooth manifold, $Y$ is a topological manifold, and correspondences $X \leftarrow\left(Z_{1}, E_{1}\right)$ $\stackrel{g}{\rightarrow} M \stackrel{f}{\leftarrow}\left(Z_{2}, E_{2}\right) \rightarrow Y$. One will get a correspondence $X \leftarrow(Z, E) \rightarrow Y$ by defining $Z$ as the fibered product $Z_{1} \times{ }_{M} Z_{2}$, and $E=\operatorname{pr}_{1}^{*} E_{1} \times \operatorname{pr}_{2}^{*} E_{2}$. For $Z$ to be a manifold, one has to assume that the maps $f$ and $g$ are smooth and transverse (i.e., $\left.\quad V\left(x_{1}, x_{2}\right) \in Z, d f T_{x_{2}}\left(Z_{2}\right)+d g T_{x_{1}}\left(Z_{1}\right)=T_{f\left(x_{2}\right)} M\right)$.

The homotopy invariance of both $g$ ! and $f_{*}$ shows that this assumption of transversality is not harmful recalling the following (cf. [15], §III)

3.1. Lemma. Let $Z_{1}, Z_{2}, M$ be smooth manifolds, $g: Z_{2} \rightarrow M$ a proper smooth map, then the subset of $C^{\infty}\left(Z_{1}, M\right)$ off's which are transverse to $g$ is a dense $G_{\delta}$.

Having defined $Z=Z_{1} \times_{M} Z_{2}$ as above, note that $\mathrm{pr}_{1}: Z \rightarrow Z_{1}$ is proper (since $f$ is proper) so that the composition $f_{X}: Z \rightarrow Z_{1} \rightarrow X$ is proper. To end the construction of $\left(Z, E, f_{X}, g_{Y}\right)$ with $g_{Y}$ the composition $Z \rightarrow Z_{2} \rightarrow Y$ one has to exhibit the $K$-orientation of $g_{Y}$, i.e. (Prop. B. 6 (c)) of pr $_{2}$. One has $T_{\mathrm{pr}_{2}}=T^{*} Z+$ $\left(\mathrm{pr}_{2}\right)^{*} T Z_{2}$ so that 


$$
T_{\mathrm{pr}_{2}} \times_{Z}\left(f \circ \mathrm{pr}_{2}\right)^{*} T_{\mathrm{Id}_{\mu K}}=\operatorname{prr}_{1}^{*} T_{g} \times_{Z} \operatorname{pr}_{2}^{*} T_{\mathrm{Id}_{Z_{2}}}
$$

which (since $g$ is $K$-oriented) gives a $K$-orientation for $\mathrm{pr}_{2}$.

3.2. Theorem. The Kasparov product $\left[Z_{1}, E_{1}\right] \otimes_{M}\left[Z_{2}, E_{2}\right]$ is given by the composition $[Z, E]$ of the correspondences.

(The brackets around a correspondence indicate the associated element of the analytical group).

Proof. Using Theorem 2.6, the problem easily reduces to show that

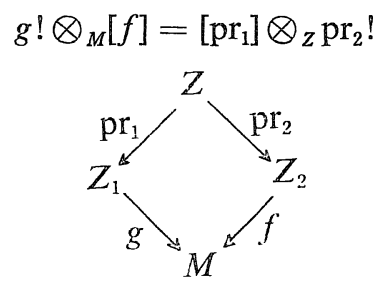

Replace $M$ by $M \times \mathbb{R}^{2 n}, Z_{2}$ by $Z_{2} \times \mathbb{R}^{2 n}, f$ by $f \times \operatorname{id}_{R^{2 n}}$ and $g$ by $(g, i)$ where $i$ : $Z_{1} \rightarrow \mathbb{R}^{2 n}$ is an embedding. Then $Z$ does not change and the two sides of the equality we want to prove are replaced by their (external) tensor product (over $\mathbb{C})$ by the Bott element $\beta \in K K\left(\mathrm{pt}, \mathbb{R}^{2 n}\right.$ ) (use Remark 2.10 (b)). So by Bott periodicity (i.e. the invertibility of $\beta$, cf. [19], Theorem $7, \S 5$ ) we are reduced to the case when $g$ is an embedding. Note then that the normal bundle $N_{2}$ of $\mathrm{pr}_{2}$ is equal to $\operatorname{pr}_{1}^{*} N$ where $N$ is the normal bundle of $g$. Using Proposition 2.8, to compute $g$ ! and $\mathrm{pr}_{2}$ !, the equality follows from the naturality of the Thom isomorphism.

3.3. Remark. Let $(Z, E),\left(Z^{\prime}, E^{\prime}\right)$ be correspondences between $X, Y \times M$ and $M \times X^{\prime}, Y^{\prime}$ then the Kasparov product $[Z, E] \times{ }_{M}\left[Z^{\prime}, E^{\prime}\right] \in K K\left(X \times X^{\prime}, Y \times Y^{\prime}\right)$ is given by the composition of the correspondences $\left(Z \times X^{\prime}, E\right)$ between $X \times X^{\prime}$, $Y \times M \times X^{\prime}$ and $\left(Y \times Z^{\prime}, E^{\prime}\right)$ between $Y \times M \times X^{\prime}, Y \times Y^{\prime}$. To see this, one uses [19] and Remark 2.10 (b).

To define the composition of the correspondences $X \leftarrow Z_{1} \stackrel{g}{\rightarrow} M$ and $M \stackrel{f}{\leftarrow} Z_{2}$ $\rightarrow Y$, one first has to make $f$ and $g$ smooth and transverse by a small $C^{0}$ perturbation. A natural question is then to see how the correspondence $Z_{1} \times{ }_{M} Z_{2}=$ $\left\{\left(x_{1}, x_{2}\right), g\left(x_{1}\right)=f\left(x_{2}\right)\right\}$ depends upon the above modification of $f$ and $g$. By Theorem 3.2 the above correspondences deine the same element of $K K(X, Y)$.

Let $f^{\prime}, g^{\prime}, f^{\prime \prime}, g^{\prime \prime}$ be such modifications, by construction there exist smooth homotopies 


$$
\left(f_{t}\right) \in C^{\infty}\left(Z_{2} \times[0,1], M\right),\left(g_{t}\right) \in C^{\infty}\left(Z_{1} \times[0,1], M\right)
$$

with $f_{0}=f^{\prime}, g_{0}=g^{\prime}, f_{1}=f^{\prime \prime}, g_{1}=g^{\prime \prime}$. But it may be impossible to ensure the transversality of $f_{t}$ and $g_{t}$ for all $t$, and to construct a homotopy between the correspondences $Z^{\prime}$ and $Z^{\prime \prime}$. However $Z^{\prime}$ and $Z^{\prime \prime}$ are cobordant in the following sense $\mathrm{e}^{(*)}$ :

3.4. Definition. Two correspondences $\left(Z^{\prime}, E^{\prime}\right),\left(Z^{\prime \prime}, E^{\prime \prime}\right)$ between $X$ and $Y$ are cobordant iff there exists a "correspondence with boundary" $(Z, E)$ such that

$$
\begin{gathered}
\partial Z=Z^{\prime} \cup Z^{\prime \prime},\left.E\right|_{Z^{\prime}}=E^{\prime},\left.E\right|_{Z^{\prime \prime}}=E^{\prime \prime}, \quad f_{X}^{\prime}=f_{\left.X\right|_{Z^{\prime}}}, \\
f_{X}^{\prime \prime}=f_{\left.X\right|_{Z^{\prime \prime}}}, \quad g_{Y}^{\prime}=g_{\left.Y\right|_{Z^{\prime}}}, \quad g_{Y}^{\prime \prime}=-g_{\left.Y\right|_{Z^{\prime}} ^{\prime \prime}}
\end{gathered}
$$

(the same map with the opposite K-orientation).

In this definition the restriction $\partial g$ of $g$ to the boundary of the manifold $Z$ is $K$-oriented by the equality $\left(\tau_{\partial g} \times_{\partial z} \nu\right)=i * \tau_{g}$, where $\nu$ is the (inward) normal bundle of $\partial Z$ while $i: \partial Z \rightarrow Z$ is the inclusion.

3.5. Proposition. Two cobordant correspondences define the same analytical element.

Proof. Let us take the notations of Definition 3.4, let $D=\left(Z \cup_{\partial Z} Z\right)$ be the double of $Z, \delta: Z \rightarrow D$ the inclusion, $p: D \rightarrow Z$ the projection. To show that the correspondence $\left(\partial Z, i^{*} E\right)$ gives $0 \in K K(X, Y)$ it is enough, using the following diagram, to show that $[\delta \circ i] \otimes_{\partial z}(\delta \circ i) !=0$.

$$
X \stackrel{f \circ p}{\longleftarrow} D \stackrel{\delta \circ i}{\longleftarrow} \partial Z \stackrel{\delta \circ i}{\longrightarrow} D \stackrel{g \circ p}{\longrightarrow} Y .
$$

Using the triviality of the normal bundle of $\partial Z$ in $Z$, construct $i^{\prime}: \partial Z \rightarrow Z$ homotopic to $i$ with $i^{\prime}(\partial Z) \cap \partial Z=\phi$. It suffices to show that $[i] \otimes_{\partial Z}\left(i^{\prime}\right) ! \in$ $K K^{1}(Z, \stackrel{\circ}{Z})$ is the 0 -element. As an element of $K K^{1}(\partial Z, \stackrel{\circ}{Z}), i^{\prime} !$ is given by the exact sequence $0 \rightarrow C_{0}(\stackrel{\circ}{Z}) \rightarrow C_{0}(Z) \rightarrow C_{0}(\partial Z) \rightarrow 0$ and hence $[i] \otimes_{\partial Z}\left(i^{\prime}\right)$ ! is given by the split exact sequence:

$$
0 \rightarrow C_{0}(\stackrel{\circ}{Z}) \rightarrow C_{0}(D) \rightarrow C_{0}(Z) \rightarrow 0 .
$$

Another way of showing that $[\delta \circ i] \otimes_{\partial z}(\delta \circ i) !=0$ is to see that this element of $K K(D, D)$ is of the form $j(U)$ for $U \in K^{1}(D)$ equal to $(\delta \circ i) ! \otimes_{D} \mathfrak{U}$ where $\mathfrak{U}$ is the trivial one dimensional complex bundle, $\mathfrak{U} \in K^{0}(\partial Z)$. One then computes $U$ and shows that its class is 0 .

Let us also remark that one can easily interpret as a correspondence, the

(*) Using for instance [15], Lemma 1.2, p.170, with $A$ the diagonal in $M \times M$. 
element of $K K^{1}(W, V \backslash W)$ given by the exact sequence: $0 \rightarrow C_{0}(V \backslash W) \rightarrow C(V)$ $\rightarrow C(W) \rightarrow 0$ where $W$ is a submanifold of $V$.

Let $S$ be the unit sphere of the normal bundle of $W$ in $V, \pi$ the projection of $S$ on $W$ and $i$ the imbedding of $S$ in $V \backslash W$ via the tubular neighborhood construction.

3.6. Proposition. The class in $K K^{1}\left(C(W), C_{0}(V \backslash W)\right)$ of the exact sequence $0 \rightarrow C_{0}(V \backslash W) \rightarrow C(V) \rightarrow C(W) \rightarrow 0$ is equal to the class $[\varepsilon]$ of the correspondence:

$$
W \stackrel{\pi}{\leftarrow} S \stackrel{i}{\rightarrow} V \backslash W
$$

Proof. Let $i$ be an identification of $N$ with a tubular neighborhood of $W$ in $V$. Let $\rho$ be the completely positive lifting of the homomorphism of restriction: $C(V) \rightarrow C(W)$, given by the conditions: $\rho(f) \in C_{0}(N) \subset C(V)$ and:

$$
\rho(f)(x, \xi)=\chi(\|\xi\|) f(x), \quad \forall(x, \xi) \in N
$$

where $\chi(t)=1-t / 2, t \in[0,2], \chi(t)=0, V t>2$. Applying the generalized Stinespring construction to $\rho([18]$, Theorem 3$)$ gives a Kasparov bimodule $(\mathcal{E}, F)$ whose class in $K K^{1}(W, V \backslash W)$ is that of the above exact sequence. One has $\mathcal{E}=C_{0}(N)$ with the obvious $C^{*}$ module structure over $C(V)$, the action of $C(W)$ on the left is given $(f \xi)(n)=f(\pi(n)) \xi(n), \forall f \in C(V), \xi \in C_{0}(N)$ (where $\pi$ : $N \rightarrow V$ is the projection). Finally the endomorphism $F$ is given by the multiplication operator:

$$
(F \xi)(x, \xi)=(2 \chi-1)(|| \xi \mid), \quad \forall(x, \xi) \in N
$$

It is now clear that if we let $i$ be the inclusion of $S=\{(x, \xi) \in N,\|\xi\|=1\}$ in $V$, one has exactly $[(\mathcal{E}, F)]=\left(i_{\text {im }}\right)$ !, hence the result follows using Proposition 2.8 .

We shall now apply Theorem 3.2 to formally deduce the analytic form of the Poincaré duality. Let $V$ be a compact smooth manifold. Let $\sigma_{V}$ be the correspondence between pt and $V \times T V$ given by

$$
\mathrm{pt} \leftarrow V \stackrel{\Delta}{\rightarrow} T V \times V \quad(\Delta(x)=((x, 0), x))
$$

$\left(\Delta\right.$ is an immersion whose normal bundle is complex ${ }^{(*)}$ so that it is naturally $K$-oriented). Let $\rho_{V}$ be the correspondence between $V \times T V$ and pt, given by: $V \times T V \stackrel{\delta}{\leftarrow} T V \rightarrow \mathrm{pt}$, where $T V$ is $K$-oriented as in Proposition B.6 (a), $\delta(x, \xi)=$

(*) Given $x \in V, \xi+i \eta \in T_{x}(V) \otimes C$, one puts $\widetilde{\Delta}(\xi)=\left(\exp _{x}(\xi), 0, x\right), \tilde{\Delta}(i \eta)=(x, \eta, x)$. 
$(x,(x, \xi))$.

3.7. Corollary. One has:

a) $\left[\sigma_{V}\right] \otimes_{V}\left[\rho_{V}\right]=1_{T V},\left[\sigma_{V}\right] \otimes_{T V}\left[\rho_{V}\right]=1_{V}$.

b) For any $C^{*}$-algebras $A, B$ the Kasparov product by $\left[\sigma_{V}\right]$ gives natural isomorphisms

$K K(A \otimes C(V), B) \rightarrow K K\left(A, B \otimes C_{0}(T V)\right), K K\left(A \otimes C_{0}(T V), B\right) \rightarrow K K(A, B \otimes C(V))$.

Proof. a) Follows from a direct computation of the composition of these correspondences using Remark 3.3.

b) Follows from a) and [19 (Theorem 6, §4)].

The next corollary shows first (a), b)) the naturality of the Poincare duality and then (c), d)) that the functors $f \rightarrow[f]$ and $f \rightarrow f$ ! are Poincaré dual.

3.8. Corollary. Let $V$ and $W$ be smooth compact manifolds, $f: V \rightarrow W$ a continuous map, $d f=T V \rightarrow T W$ a continuous extension of $f$. One has:

a) $\left[\sigma_{V}\right] \otimes_{T V}(d f) !=\left[\sigma_{W}\right] \otimes_{W}[f]$.

b) $(d f) ! \otimes_{T W}\left[\rho_{W}\right]=[f] \otimes_{V}\left[\rho_{V}\right]$.

c) $(d f) !=\left[\sigma_{W}\right] \otimes_{W}[f] \otimes_{V}\left[\rho_{V}\right]$.

d) $\left[\sigma_{V}\right] \otimes_{T V}(d f) ! \otimes_{T W}\left[\rho_{W}\right]=[f]$.

Proof. From Corollary 3.7 a) all these equalities are equivalent. To prove a) one again computes directly the composition of these correspondences (using Remark 3.3).

In [20] G.G. Kasparov proves the Poincaré duality in a very similar way.

3.9. Remark. Let Cliff $V=\operatorname{Cliff}(V, T V)$ be the graded $C^{*}$-algebra of continuous sections of the bundle of Clifford algebras $\mathrm{Cliff}_{C} T_{x}$. The Thom isomorphism [19], Theorem 8, $\$ 5$ gives two elements $\beta \in K K($ Cliff $V, T V), \alpha \in$ $K K(T V$, Cliff $V)$ such that $\alpha \otimes \beta=1_{T V}, \beta \otimes \alpha=1_{\text {Cliff } V}$.

One can translate Corollaries 3.7 and 3.8 replacing $C_{0}(T V)$ by Cliff $V$, using

$$
\tilde{\rho}_{V}=\beta \otimes_{T V} \rho_{V} \in K K(C(V) \otimes \text { Cliff } V, \mathbb{C})
$$

and $\tilde{\sigma}_{V}=\sigma_{V} \otimes_{T V} \alpha \in K K(\mathbb{C}$, Cliff $V \otimes C(V))$. In fact $\tilde{\rho}_{V}$ is equal to the following element of $K K(C(V) \otimes C$ liff $V, \mathbb{C})$. The Hilbert space is the space of $L^{2}$ sections of the complex vector bundle over $V$ with fiber $\operatorname{Cliff}_{C}\left(T_{x}(V)\right.$ ). Both $C(V)$ and Cliff $V$ act by (left) multiplication operators and the operator $F$ is an ordinary pseudo-differential operator of order 0 with principal symbol $\sigma(x, \xi)=\operatorname{ir}(\xi) \varepsilon$, 
$\xi \in T^{*} V,\|\xi\|=1$, where $r(\xi)$ is the right Clifford multiplication by $\xi, \varepsilon$ is the grading of $\operatorname{Cliff}_{C}\left(T_{x}(V)\right)$.

To see that these two descriptions of $\tilde{\rho}_{V}$ coincide, one checks that the Kasparov product $\alpha \otimes_{\text {cliff } V} \tilde{\rho}_{V}$ is (for both of them) equal to $\rho_{V}$.

The next proposition shows the meaning of the Poincaré duality

$$
K^{*}(T V) \stackrel{\otimes \rho_{V}}{\longrightarrow} K K(V, \mathrm{pt}), K K(V, \mathrm{pt}) \stackrel{\sigma_{V} \otimes}{\longrightarrow} K^{*}(T V)
$$

as associating to a symbol a pseudo-differential operator and vice versa.

3.10. Proposition. a) Let $\sigma \in K K\left(\mathrm{pt}, T^{*} V \times Y\right)$ ( $Y$ a locally compact space), then $[\sigma] \otimes_{T^{*} V}\left[\rho_{V}\right]=\Psi^{*}(\sigma) \in K K(V, Y)($ cf. Section $I)$.

b) Let $P \in K K(V, Y)$ then $P=\Psi^{*}(\sigma)$, with $[\sigma]=\sigma_{V} \bigotimes_{V} P \in K^{*}(T V \times Y)$.

Proof. a) Let $S$ be a (graded) $\operatorname{Cliff}(V) \otimes C_{0}(Y)$ module corresponding to $[\sigma]$ through the Thom isomorphism. One has

$$
[\sigma] \otimes_{T^{*} V}\left[\rho_{V}\right]=[\sigma] \otimes \alpha \otimes \tilde{\rho}_{V}=S \otimes \tilde{\rho}_{V}
$$

which (using the second description of $\tilde{\rho}_{V}$ ) is the family (indexed by $Y^{(*)}$ ) of Dirac operators on $V$ with coefficients in $S$. On the other hand, as $[\sigma]=\beta \otimes[S]$ it is clear that $P_{\sigma}$ is also the family of Dirac operators on $V$ with coefficients in $S$.

b) Follows from a) and Corollary 3.8.

3.11. Corollary. The map $\Psi^{*}$ from $K^{*}\left(T^{*} V \times Y\right)$ to $K K^{*}(V, Y)$ of Section $I$ is bijective. In particular any element of $K^{*}(V, Y)$ is given by a family of pseudo-differential operators on $V$.

Combining Proposition 3.10 with the multiplicativity (Theorem 2.6), one gets the Atiyah-Singer index theorem (see [1]). Indeed let $P_{\sigma}$ be an elliptic pseudo-differential operator on $V,\left[P_{\sigma}\right] \in K K(V, \mathrm{pt})$ its class and $\pi: V \rightarrow \mathrm{pt}$ the obvious map. Then the analytical index of $P_{\sigma}$ is obviously

$$
\pi_{*}\left[P_{\sigma}\right]=[\pi] \otimes_{V}\left[P_{\sigma}\right] \in K K(\mathrm{pt}, \mathrm{pt}) .
$$

The topological index of $P_{\sigma}([1])$ is defined using an embedding $j: V \rightarrow \mathbb{R}^{n}$ by $[\sigma] \otimes_{T V}(d j) !_{\mathrm{im}} \otimes_{T R^{n}}(d p) !\left(\right.$ with $\left.p: \mathbb{R}^{n} \rightarrow \mathrm{pt}\right)$. One has

$$
\begin{aligned}
& \operatorname{Ind}_{a}\left(P_{\sigma}\right)=[\pi] \bigotimes_{V}\left[P_{\sigma}\right]=[\pi] \bigotimes_{V}\left([\sigma] \otimes_{T^{*} V}\left[\rho_{V}\right]\right) \\
= & {[\sigma] \otimes_{T^{*} V}\left([\pi] \bigotimes_{V}\left[\rho_{V}\right]\right)=[\sigma] \bigotimes_{T V}(d \pi) ! . }
\end{aligned}
$$

(*) We assume $Y$ compact to simplify, replacing it by the one point compactification $Y^{+}$. 
As $\pi=p \circ j$ the equality $\operatorname{Ind}_{a}\left(P_{\sigma}\right)=\operatorname{Ind}_{t}\left(P_{\sigma}\right)$ follows from Theorem 2.6.

Combining Proposition 3.10 with Theorem 3.2., one gets the odd index theorem, cf. [17] and [6], Theorem 24.3. Indeed let $\mu: K_{1}^{t}(V) \rightarrow K_{1}^{a}(V)=$ $K K^{1}(V, p t)$ be the map defined in [6], (18.1), p. 36 from the topological group to be the analytical group. In our notation $\mu\left(M, E_{0}, f\right)=f_{*}\left(\left(E_{0}\right) \otimes_{M} p\right.$ !) where $p$ is the map: $M \rightarrow$ pt. Let $E$ be a complex vector bundle on the smooth compact manifold $V, \sigma: \pi^{*}(E) \rightarrow \pi^{*}(E)$ a self-adjoint Clifford symbol, $A=P_{\sigma}$ a corresponding self-adjoint order 0 pseudo-differential operator on $V$ (from $E$ to $E)$. Then by Proposition 3.8, $[A] \in K_{1}^{a}(V)=K K^{1}(V, \mathrm{pt})$ is equal to $[\sigma] \otimes_{T^{*} V} \rho_{V}$. Put $M=S^{*} V, E_{0}=E^{+}$(cf. [6], p. 48, (24.1)), $f=\pi: S^{*} V \rightarrow V$, so that Theorem 24.3 of [6] is the equality $\pi_{*}\left(\left(E^{+}\right) \otimes_{S^{*} V} p_{S^{*} V} !=[\sigma] \otimes_{T^{*} V} \rho_{V}\right.$. This result follows using the following diagram, from Theorem 3.2 and the equality $\left[E^{+}\right] \otimes_{S^{*} V} i$ ! $=[\sigma] \in K K^{1}\left(\mathrm{pt}, T^{*} V\right)$ where $i: S^{*} V \rightarrow T^{*} V$ is the inclusion.

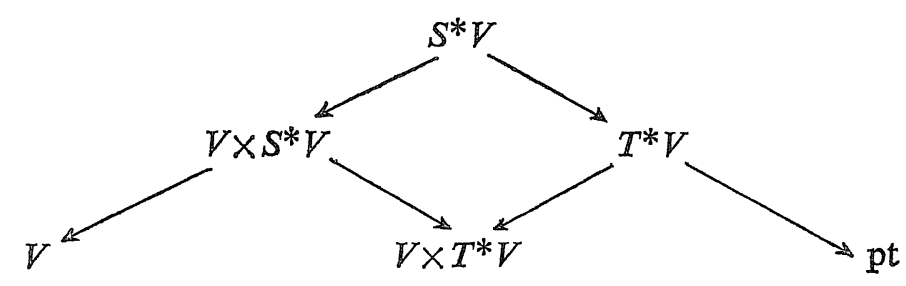

$\S \mathbb{E}$. The Longitudinal Hndex Theorem

Given a foliation $(V, F)$ and a $K$-oriented map $f$ from a manifold $W$ to the space of leaves $V / F$, we associate an element $f !$ of $K K(W, V / F)\left(\stackrel{\text { 'def }}{=} K K\left(C_{0}(W)\right.\right.$, $\left.C^{*}(V, F)\right)$ ). We first discuss this contruction when $f$ is a submersion. The general case is then obtained by factorizing the map $f$ through a submersion $\pi(W \stackrel{j}{\rightarrow} X \stackrel{\pi}{\rightarrow} V \mid F)$.

The longitudinal index theorem is then obtained, considering the map $V \rightarrow V / F$, and factorizing it in different ways.

The element $f$ ! corresponding to a $K$-oriented submersion $f: W \rightarrow V / F$ is naturally written as the Kasparov product of two elements:

1) An element $p_{W} ! \in K K\left(W, W / F_{W}\right)$ where $F_{W}$ is the pull back foliation by $f$ in $W$ and $p_{W}: W \rightarrow W / F_{W}$ is the natural projection.

2) A quite trivial element $\varepsilon_{f} \in \mathbb{K} K\left(W / F_{W}, V / F\right)$ which is given by a Hilbert $C^{*}(V, F)$ module $\varepsilon_{f}$ on which $C^{*}\left(W, F_{W}\right)$ acts by compact operators.

The element $p_{W}$ ! will be constructed using the extension of pseudo-differential 
operators of [9] (§IX, p. 138).

The main fact about this construction is the functoriality $(f \circ g) !=g ! \bigotimes_{W} f !$ where $g: X \rightarrow W, f: W \rightarrow V \mid F$ are $K$ oriented submersions. This will allow us to prove that for a general ( $K$ oriented) $f: W \rightarrow V / F$ the element $j ! \otimes \pi !$ does not depend upon the factorization

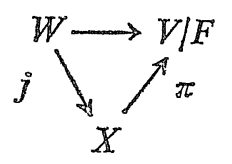

where $\pi$ is a submersion, and hence to define $f$ ! for general maps by $f !=j ! \otimes \pi$ !.

We first construct the element $\varepsilon_{f} \in K K\left(W / F_{W}, V / F\right)$ corresponding to a smooth submersion $W \rightarrow V / F$. Let us fix some notations (see [13], [10]).

Let $V, F$ be a foliated manifold and let $G$ be the graph of the foliation i.e., the holonomy groupoid ([26] or also [9], §VII).

4.1. Definition. 1. A map $f: W \rightarrow V / F$ is given by its graph which is a principal (right) $G$ bundle $G_{f}$ over $W$. We call $r_{f}: G_{f} \rightarrow W$ and $s_{f}: G_{f} \rightarrow G^{(0)}=V$ the corresponding maps.

2. The map $f: W \rightarrow V / F$ is called a submersion if the map $s_{f}$ is a submersion.

For $\alpha \in G_{f}$ and $r \in G$ with $s_{f}(\alpha)=r(\gamma)$ the action of $\gamma$ on $\alpha$ is noted $\alpha \circ \gamma$. Recall that we have $s_{f}(\alpha \circ \gamma)=s(\gamma), r_{f}(\alpha \circ \gamma)=r_{f}(\alpha)$ and if $r_{f}\left(\alpha_{1}\right)=r_{f}\left(\alpha_{2}\right)$ there exists a unique $r \in G$ with $\alpha_{2}=\alpha_{1} \circ \gamma$ ( $G_{f}$ is principal).

Recall also that an equivalent way of giving the map $f$ is a cocycle $\left(\Omega_{i}, \gamma_{i j}\right)$ on $W$ with values in $G\left(\Omega_{i}\right.$ is an open covering of $W$ and $\gamma_{i j}: \Omega_{i} \cap \Omega_{j} \rightarrow G$ satisfies

$$
r_{i j}(x) \circ \gamma_{j k}(x)=r_{i k}(x) \quad\left(\forall x \in \Omega_{i} \cap \Omega_{j} \cap \Omega_{k}\right) .
$$

Recall (cf. [10]) that given the cocycle $\left(\Omega_{i}, \gamma_{i j}\right), G_{f}$ is obtained by gluing together the open sets $\widetilde{\Omega}_{i}=\left\{(x, \gamma) \in \Omega_{i} \times G, \gamma_{i i}(x)=r(\gamma)\right\}$ by the map $\tilde{\Omega}_{i} \rightarrow$ $\tilde{\Omega}_{j},(x, \gamma) \rightarrow\left(x, r_{j i}(x) \circ \gamma\right)$. One has

$$
r_{f}(x, \gamma)=x, \quad s_{f}(x, \gamma)=s(\gamma) \quad\left(\text { if }(x, \gamma) \in \tilde{\Omega}_{i}\right)
$$

The map $f$ is a submersion iff $\gamma_{i i}: \Omega_{i} \rightarrow V$ are transverse to the foliation $F$ (i.e., $d_{\gamma_{i i}} T \Omega_{i}+F=T V$, cf. [22], p. 378).

Let $f: W \rightarrow V / F$ be a submersion. Call $\tau=\mathbb{T V} / F$ the transverse bundle on $V$ and $\pi: T V \rightarrow \tau$ the projection. Then the subbundle of $T W, \mathbb{K e r} \pi \circ d_{\boldsymbol{\gamma}_{i i}}$ is well defined (it does not depend upon $i$ ) and is integrable. Hence it defines the pull 
back foliation $f^{*}(F)=F_{W}$ in $W$ (cf. [22], p. 373).

Let $G_{W}$ be the graph of this foliation. Then $G_{W}$ acts naturally and freely on $G_{f}$, in the following way:

4.2. Lemma. a) Let $w(t), t \in[0,1]$ be a path drawn in a leaf of $W$. Let $\alpha \in G_{f}$ with $r_{f}(\alpha)=w(0)$. Then there exists a unique path $\alpha(t)$ in $G_{f}$ satisfying

$$
\alpha(0)=\alpha, \quad r_{f}\left(\alpha_{t}\right)=w(t), \quad s_{f}\left(\alpha_{t}\right)=s_{f}(\alpha) .
$$

b) $\alpha(1)$ only depends upon the holonomy of the path $w$. Moreover, if $\alpha(1)=\alpha$, $w$ is a loop with trivial holonomy.

If $\gamma$ is the class of $W$ in $G_{W}$ one puts $r \circ \alpha=\alpha(1)$. This lemma proves that this action is well defined and free.

Proof. a) Any path $w$ can be lifted in a path $\beta$ with $\beta(0)=\alpha$ and $r_{f}(\beta(t))=$ $w(t)$, consider then the path $s_{f}(\beta(t))$. It is drawn in the leaf of $s_{f}(\alpha)$ in $V$. Call then $r_{t}$ the class in $G$ of the path $s_{f}(\beta(u)) 0 \leqq u \leqq t$, and put $\alpha(t)=\beta(t) \circ \gamma(t)$.

The uniqueness of the lifting is given by the fact that if $\alpha$ and $\alpha^{\prime}$ are two liftings: as $r_{f}(\alpha(t))=r_{f}\left(\alpha^{\prime}(t)\right), \alpha(t)=\alpha^{\prime}(t) \circ \gamma(t)$ where $r(t) \in G$, but $r(\gamma(t))=s(\gamma(t))$ $=s_{f}(\alpha)$ hence $r$ is constant and

$$
r(t)=s_{f}(\alpha) \in V=G^{(0)}(\subset G) .
$$

b) It is enough to show that given a loop $w$ the corresponding $\alpha(1)$ is equal to $\alpha$ if and only if the holonomy of $w$ is trivial. As $G_{f}$ is a $G$ principal bundle there exists a unique $r \in G$ with $\alpha(1)=\alpha \circ \gamma$. Let $r(t), t \in[0,1]$ be a path in $G$ joining $s_{f}(\alpha)$ with $r^{-1}$, with $r(\gamma(t))=s_{f}=r(\gamma)=s(\gamma)$. Put then $\beta(t)=\alpha(t) r(t)$. It is a loop drawn in a leaf of $G_{f}$ (which is foliated by the pull back foliation of any of the maps $r_{f}$ or $s_{f}$ ). This loop has the same holonomy as its two projections in $V$ and $W$. Thus $r$ has the same holonomy as the loop $w(t)$.

Let us now define the Hilbert $C^{*}(V, F)$ module $\varepsilon_{f}$ :

It is the completion of $C_{c}\left(G_{f}, \Omega^{1 / 2}\right)^{(*)}$ with respect to its $C_{c}\left(G, \Omega^{1 / 2}\right)^{(*)} \subset$ $C^{*}(V, F)$ valued inner product

$$
\langle\xi, \eta\rangle(\gamma)=\int_{\infty \circ \gamma=\beta} \overline{\xi(\alpha)} \eta(\beta)
$$

The right module structure is given by:

$$
(\xi h)(\alpha)=\int_{\beta \circ \gamma=\alpha} \xi(\beta) h(\gamma), \quad \xi \in C_{c}\left(G_{f}, \Omega^{1 / 2}\right), \quad h \in C_{c}\left(G, \Omega^{1 / 2}\right)
$$

(*) Where $\Omega^{1 / 2}$ is a suitable $1 / 2$ density bundle (cf. [9], $\S$ VII and Remark 1.1.1). 
The left $C_{c}\left(G_{W}, \Omega^{1 / 2}\right)$ action on $C_{c}\left(G_{f}, \Omega^{1 / 2}\right)$ is given by:

$$
\pi_{f}(g) \xi(\alpha)=\int_{\gamma \circ \beta=\infty} g(\gamma) \xi(\beta)
$$

4.3. Proposition. a) This action extends to an injective *-homomorphism $\pi_{f}: C^{*}\left(W, F_{W}\right) \rightarrow \mathbb{R}\left(\mathcal{E}_{f}\right)$.

b) Moreover $\pi_{f}\left(C^{*}\left(W, F_{W}\right)\right) \subseteq \Omega\left(\varepsilon_{f}\right)$.

c) $\pi_{f}\left(C^{*}\left(W, F_{W}\right)\right) \mathcal{E}_{f}$ is dense in $\mathcal{E}_{f^{\circ}}$

Proof. a) The elements of $C^{*}\left(W, F_{W}\right)$ are families, indexed by the space of leaves of $W$, of operators $P_{L}, P_{L} \in \mathbb{R}\left(L^{2}(\tilde{L})\right)(\tilde{L}$ is the holonomy covering of $L([10], \S 7)$. The elements of $\mathcal{L}\left(\mathcal{E}_{f}\right)$ are families, indexed by the space of leaves of $V$, of operators $Q_{L}, Q_{L} \in \mathfrak{R}\left(\mathfrak{S}_{L}\right)$ where $\mathfrak{S}_{L}=\mathbb{L}^{2}\left(G_{f, x}\right)(x \in L), G_{f, x}=s_{f}^{-1}(\{x\})$. As $G_{W}$ acts freely on $G$ each $G_{f, x}$ is partitioned in holonomy coverings of leaves of $W$ (those are in fact the connected components of $G_{f, x}$ ). Hence for $g \in C_{c}\left(G_{W}, \Omega^{1 / 2}\right)$

$$
\operatorname{Sup}_{L_{W} \in W / F_{W}}\left\|P_{L_{W}}\right\|=\operatorname{Sup}_{L_{V} \in V / F}\left\|Q_{L_{V}}\right\|
$$

where $P_{L_{W}}$ is the family corresponding to $g$, and $Q_{L_{V}}$ the one corresponding to $\pi_{f}(g)$. Thus

$$
\|g\|=\left\|\pi_{f}(g)\right\|
$$

b) Let $\Omega \subset W$ be a small enough open subset of an $\Omega_{i}$ such that $r_{i i}(\Omega) \subset \Omega^{\prime}$, where $\Omega$ and $\Omega^{\prime}$ are foliation charts. Assume also that the induced map from $\Omega / F_{W}$ to $\Omega^{\prime} / F$ is injective (reducing again $\Omega$ if necessary; here $\Omega / F_{W}$ represents the space of plaques of $\Omega$ ).

Let $G_{\Omega^{\prime}} \subset G$ (resp. $G_{\Omega} \subset G_{W}$ ) be the graph of the foliation $F$ (resp. $F_{W}$ ) restricted to $\Omega^{\prime}\left(\right.$ resp. $\Omega$ ). Let $\Omega^{\prime \prime} \subset \widetilde{\Omega}_{i} \subset G_{f}, \Omega^{\prime \prime}=\left\{(x, \gamma) ; x \in \Omega, \gamma \in G_{\Omega^{\prime}}, r(\gamma)=\right.$ $\left.\gamma_{i i}(x)\right\} . \quad \Omega^{\prime \prime}$ is an open subset of $G_{f}$.

If $\alpha, \beta \in \Omega^{\prime \prime}$ satisfy $s(\alpha)=s(\beta)$, there exists a unique $\gamma \in G_{\Omega}$ such that $\gamma \circ \beta=\alpha$. Call it $\alpha \circ \beta^{-1}$. $\xi(\alpha) \overline{\eta(\beta)}$.

For $\xi, \eta \in C_{c}\left(\Omega^{\prime \prime}, \Omega^{1 / 2}\right) \subset \mathcal{E}_{f}, \quad \theta_{\xi, \eta}=\pi_{f}\left(\xi * \eta^{*}\right)$ where $\xi * \eta *(\gamma)=\int_{\alpha^{\circ} \beta^{-1}=\gamma}$

Thus $\pi_{f}\left(C^{*}\left(\Omega, F_{W}\right)\right) \subset \Omega\left(\mathcal{E}_{f}\right)$; but these algebras generate $C^{*}\left(W, F_{W}\right)$.

c) Let $h_{n} \in C_{c}\left(G_{W}, \Omega^{1 / 2}\right)$ be a sequence satisfying:

1) $h_{n}(\gamma) \geq 0, \forall r$,

2) $\operatorname{Sup}\left\{l(\gamma), r \in\right.$ Support $\left.h_{n}\right\} \rightarrow 0$ when $n \rightarrow \infty$ ( $l$ is the length of $\gamma$ )

3) (for a given trivialisation of the bundle $\Omega^{1 / 2}$ ) $\int_{\gamma(\gamma)=x} h_{n}(\gamma) \rightarrow 1$ uniformly 
on compact sets.

Then, obviously $\pi_{f}\left(h_{n}\right) \xi-\xi$ tends to 0 uniformly for any $\xi \in C_{c}\left(G_{f}, \Omega^{1 / 2}\right)$ and has support in a fixed compact set. Hence

$$
\left\|h_{n} * \xi-\xi\right\|_{\mathcal{E}_{f}} \rightarrow 0
$$

4.4. Corollary. The (trivially graded) bimodule $\mathcal{E}_{f}$ (together with the zero operator!) defines an element $\varepsilon_{f}$ of $K K\left(W / F_{W}, V / F\right)$.

Proof. This is a translation of b) of Proposition 4.3.

Let now $X \stackrel{g}{\rightarrow} W / F_{W}$ be another submersion. The composition $f \circ g$ is a submersion $X$ to $V / F$ which is given by its graph $G_{f^{\circ} g}=G_{g} \times G_{W} G_{f}$. It is the quotient of $\left\{(\beta, \alpha) \in G_{g} \times G_{f}, s_{g}(\beta)=r_{f}(\alpha)\right\}$ by the relation $(\beta, \alpha) \sim\left(\beta \gamma, \gamma^{-1} \alpha\right)$ for $r \in G_{W}, r(\gamma)=s_{g}(\beta)$. (Note that since $G_{g}$ is a $G_{W}$ principal bundle, the $G_{W}$ action on it is free and proper. Hence $G_{f^{\circ} g}$ makes sense as a manifold).

By construction $G_{f^{\circ} g}$ is a $G=G_{V}$ principal bundle over $X$ on which $G_{X}$ acts on the left $\left(G_{X}\right.$ is the graph of the pull back foliation $g^{-1}\left(F_{W}\right)=(f \circ g)^{-1} F$ on $\left.X\right)$. If $\tilde{g}: X \rightarrow W$ is smooth and transverse to the foliation $F_{W}$, the map $f \circ \tilde{g}$ can also be viewed as the cocycle $\left(\tilde{g}^{-1}\left(\Omega_{i}\right), \gamma_{i j} \circ \tilde{g}\right)$ or as the pull back by $g$ of the $G$ principal bundle $G_{f}$ over $W$.

4.5. Proposition. The bimodule $\mathcal{E}_{f^{\circ} \mathrm{g}}$ is canonically isomorphic to $\mathcal{E}_{g} \otimes_{C^{*}\left(W, F_{W}\right)} \mathcal{E}_{f} . \quad$ In particular,

$$
\varepsilon_{f^{\circ} g}=\varepsilon_{g} \bigotimes_{W / F_{W}} \varepsilon_{f}\left(\in K K\left(X / F_{X}, V / F\right)\right) .
$$

Proof. For $\xi \in C_{c}\left(G_{f}, \Omega^{1 / 2}\right), \eta \in C_{c}\left(G_{g}, \Omega^{1 / 2}\right)$, we let $\eta * \xi(\gamma)=\int_{\beta^{\circ} \alpha=\gamma} \eta(\beta) \xi(\alpha)$ (where $\beta \circ \alpha$ is the class in $G_{f^{\circ} g}$ of $(\beta, \alpha)$ ). Then $\eta * \xi \in C_{c}\left(G_{f^{\circ} g}, \Omega^{1 / 2}\right)$. The equality $\left\langle\eta_{1} * \xi_{1}, \eta_{2} * \xi_{2}\right\rangle=\left\langle\xi_{1},\left\langle\eta_{1}, \eta_{2}\right\rangle \xi_{2}\right\rangle$ is obvious. Hence $\eta \otimes \xi \rightarrow \eta * \xi$ is an isometry

$$
\varepsilon_{g} \otimes_{C^{*}(W, F W)} \mathcal{E}_{f} \rightarrow \varepsilon_{f^{\circ} g}
$$

To show that this map is surjective, it is enough to prove that $\pi_{f^{\circ} g}(h) \xi$ is in its image for all $h \in C^{*}\left(X, F_{X}\right)$ and $\xi \in \mathcal{E}_{f^{\circ} g}$ (Proposition 4.3 (c)). But (using Proposition 4.3 (b) applied to $\mathrm{g}$ ), it is then enough to prove that for $\eta_{1}, \eta_{2} \in \varepsilon_{g}$ such that $\theta_{\eta_{1}, \eta_{2}} \in \pi_{g}\left(C^{*}\left(X, F_{X}\right)\right)$ one has

$$
\left(\eta_{1} * \eta_{2}^{*}\right) * \xi \in \mathcal{E}_{g} \otimes \mathcal{E}_{f} .
$$

But $\eta_{2}^{*} * \xi$ makes sense obviously in $\mathcal{E}_{f}\left(\eta_{2}^{*} * \xi(\beta)=\int_{\alpha^{\circ} \beta=\gamma} \bar{\eta}_{2}(\alpha) \xi(\gamma)\right)$. One then 
uses the associativity

$$
\left(\eta_{1} * \eta_{2}^{*}\right) * \xi=\eta_{1} *\left(\eta_{2}^{*} * \xi\right)
$$

Let $(V, F)$ be a foliation, and $E$ a Hermitian $\mathbb{Z} / 2$ graded bundle on $V$.

Let us recall the exact sequence of pseudo-differential operators of ([9], p. 138, (IX)). Choose an auxiliary phase function $\varphi(\gamma, \eta)$, defined for $\gamma$ in a neighborhood of $V=G^{(0)}$ in $G$ and for $\eta \in F_{r(\gamma)}^{*}$, and locally of the form $\langle r(\gamma)-$ $s(\gamma), \eta\rangle$, and choose a smooth $\chi, \chi(\gamma) \in \mathbb{L}\left(E_{r(\gamma)} \otimes \Omega_{F}^{1 / 2}, E_{s(\gamma)} \otimes \Omega_{F}^{1 / 2}\right)$, with support contained in the domain of definition of $\varphi$, and which is the identity for $r \in G^{(0)}=V$. Let $\mathcal{E}_{V, E}$ be the $C^{*}$-module over $C^{*}(V, F)$ corresponding to the field $\mathfrak{S}_{L}=L^{2}(\tilde{L}, E), L \in V / F$. It is obtained as the completion of $C_{c}(G$, $\left.\Omega^{1 / 2} \otimes r^{*}(E)\right)$ with respect to the $C^{*}(V, F)$ valued inner product

$$
\langle\xi, \eta\rangle(\gamma)=\int_{\gamma_{1}^{-1} \gamma_{2}=\gamma}\left\langle\xi\left(\gamma_{1}\right), \eta\left(\gamma_{2}\right)\right\rangle_{E}
$$

By construction it is a left $C_{b}(V)$ module.

Let $\sigma \in C_{c}^{\infty}\left(F_{1}^{*}, \mathfrak{R}(E)\right)$, where $F_{1}^{*}$ is the space of half lines in $F^{*}$, extending it smoothly to $F^{*}$ in such a way that it coincides with $\sigma$ outside a compact set, the formula:

$$
\begin{gathered}
\left(P_{\sigma} \xi\right)\left(\gamma^{\prime}\right)=\int_{\xi \in C_{c}^{\infty}\left(G, \Omega^{i \varphi(\gamma, \eta)} \sigma(s(r), \eta) \chi(\gamma) \xi\left(r r^{\prime}\right) d r d \eta\right.}, \\
\left.r^{*}(E)\right)
\end{gathered}
$$

defines an endomorphism of the $C^{*}$-module $\varepsilon_{V, E}$.

Let $\Psi_{0}^{*}$ be the $C^{*}$-subalgebra of $\mathfrak{R}\left(\mathcal{E}_{V, E}\right)$ generated by such $P_{\sigma}{ }^{2}$ s. Then one has an exact sequence:

$$
0 \rightarrow \Re\left(\mathcal{E}_{V, E}\right) \rightarrow \Psi_{0}^{*} \stackrel{\sigma}{\rightarrow} C_{0}\left(F_{1}^{*}, \mathfrak{\Omega}(E)\right) \rightarrow 0,
$$

where the symbol map $\sigma$ is given as usually (cf. [9], p. 138). For our purpose, with $V$ non compact, we let $\Psi^{*}$ be the $C^{*}$-subalgebra of $\mathfrak{L}\left(\mathcal{E}_{V, E}\right): \Psi^{*}=\{P \in$ $\left.\mathfrak{L}\left(\mathcal{E}_{V, E}\right), P h \in \Psi_{0}^{*}, h P \in \Psi_{0}^{*}, \forall h \in C_{0}(V)\right\}$, and we let $\Re_{V}\left(\mathcal{E}_{V, E}\right)=\left\{T \in \mathcal{L}\left(\mathcal{E}_{V, E}\right), T h\right.$ $\left.\in \Re\left(\mathcal{E}_{V, E}\right), h T \in \Omega\left(\mathcal{E}_{V, E}\right), \forall h \in C_{0}(V)\right\}$. One gets:

4.6. Proposition. The following sequence is exact:

$$
0 \rightarrow \Re_{V}\left(\varepsilon_{V, E}\right) \rightarrow \Psi^{*} \stackrel{\sigma}{\rightarrow} C_{b}\left(F_{1}^{*}, \mathfrak{L}(E)\right) \rightarrow 0 .
$$

Proof. The only thing to prove is that $\sigma$ defined by the equality $\sigma(h P)=$ $h \sigma(P), h \in C_{0}(V)$ is surjective. Given $a \in C_{b}\left(F_{1}^{*}, \mathfrak{L}(E)\right), \varepsilon \geq 0$ and $h_{1}, h_{2} \in C_{c}(V)$, $0 \leq h_{1} \leq h_{2} \leq 1$ and $P_{1} \in \Psi_{0}^{*}$ with $h_{1} \sigma\left(P_{1}\right)=h_{1} a$, one can find $P_{2},\left\|P_{2}\right\| \leq \operatorname{Sup}(2\|a\|$, 
$\left.\left\|P_{1}\right\|\right)$ with $h_{2} \sigma\left(P_{2}\right)=h_{2} a$ and $\left\|\left(P_{2}-P_{1}\right) h_{1}\right\| \leq \varepsilon$. Thus, one can construct a bounded sequence $P_{n} \in \Psi_{0}^{*}$ such that $P_{n} h$ is convergent in $\Psi_{0}^{*}$ with $\sigma\left(P_{n} h\right) \rightarrow a h$ for all $h \in C_{c}(V)$. Thus $P \xi=\lim _{n \rightarrow \infty} P_{n} \xi$ exists for any $\xi \in \mathcal{E}_{V, E}=\overline{C_{c}(V) \mathcal{E}_{V, E}}$ and $P \in \Psi^{*}, \sigma(P)=a^{(*)}$.

We can now associate to a given $\operatorname{Spin}^{c}$ structure $S$ on the bundle $F$ an element $p$ ! of $K K(V, V / F)$ ( $p$ the projection $V \rightarrow V / F)$. Indeed the $C^{*}$-module $\mathcal{E}_{V, S}$ is naturally $\mathbb{Z} / 2$ graded and for any $D \in \Psi^{*}$ of degree one, with symbol $\sigma(D)(x, \xi)=c(\xi /\|\xi\|) \in$ End $S$, the pair $\left(\mathcal{E}_{V, S}, D\right)$ is a Kasparov bimodule, whose class is independent of the choice of $D$. (Note that this construction works for any Clifford symbol for the bundle $F^{*}$ and gives a canonical map of $K_{V}\left(F^{*}\right)$ to $K K(V, V / F))$.

We can now prove the crucial lemma of this section:

4.7. Lemma. Let $g: W \rightarrow V$ be a smooth K-oriented (by a $\operatorname{Spin}^{c}$ structure) submersion, let $\mathbb{F}_{W}$ be the pull back foliation on $W^{(* *)}, p_{W}: W \rightarrow W \mid F_{W}$, Then:

$$
g ! \otimes_{V} p_{V} !=p_{W} ! \otimes_{W / F_{W}} \varepsilon_{p_{V^{\circ} g}} \in K K(W, V / F)
$$

where $\varepsilon_{p_{V^{\circ} g}}$ is defined by Proposition 4.3.

Proof. Let $f=p_{V} \circ g$. Let us compute the right hand side. The bimodule $\mathcal{E}_{W, S_{W}} \otimes_{C^{*}\left(W, F_{W}\right)} \mathcal{E}_{f}$ is easily described as the completion $\mathcal{E}_{f, S_{W}}$ of $C_{c}\left(G_{f}, r_{f}^{*}\left(S_{W}\right)\right.$ $\left.\otimes \Omega^{1 / 2}\right)$ with the $C^{*}(V, F)$ valued inner product given by $\left\langle\xi_{1}, \xi_{2}\right\rangle(\gamma)=$ $\int_{\infty \circ \gamma=\beta}\left\langle\xi_{1}(\alpha), \xi_{2}(\beta)\right\rangle$. As a Kasparov bimodule, $p_{W} ! \hat{\otimes}_{W / F_{W}} \varepsilon_{f}$ is given by

$$
\left(\mathcal{E}_{W, S_{W}} \hat{\otimes}_{C^{*}\left(W, F_{W}\right)} \mathcal{E}_{f}, D_{W} \hat{\otimes} 1\right)=\left(\mathcal{E}_{f, s_{W}}, Q\right)
$$

(where $Q=\pi_{f}\left(D_{W}\right)$ ), $\pi_{f}$ is defined as in Proposition 4.3).

Recall that $G_{f}=W \times_{V} G_{V}$. By Proposition 2.9., we can describe $g$ ! by the Kasparov bimodule $\left(\mathcal{E}_{g, s_{g}}, D_{g}\right)$ corresponding to the field $\left(L^{2}\left(g^{-1}(x), S_{g}\right)\right.$, $\left.D_{g, x}\right)_{x \in V}$, where $D_{g, x}$ is a Dirac operator of order 0 on $g^{-1}(x)$. The bimodule $\mathcal{E}_{g, S_{g}} \hat{\otimes}_{C_{0}(V)} \mathcal{E}_{V, S}$ is naturally isomorphic to $\mathcal{E}_{f, S_{W}}$ by the map $i(\xi \otimes \eta)(w, r)=$ $\xi(w) \hat{\otimes} \eta(\gamma) \in\left(S_{g, w} \otimes \Omega^{1 / 2}\right) \hat{\otimes}\left(S_{V, r(\gamma)} \otimes \Omega^{1 / 2}\right)=S_{W} \otimes \Omega^{1 / 2}$.

It is hence enough to check that $Q \in$ End $\mathcal{E}_{f, S_{W}}$ is a $D_{V}$ connection and satisfies the positivity condition:

$$
\left[D_{g} \hat{\otimes} 1, Q\right] \geq 0 \quad \text { (modulo } \Omega\left(\mathcal{E}_{f, s_{W}}\right) .
$$

(*) In order to construct $P$ we have to prove that $P_{n}^{*} \xi$ also converges: In the inductive step, we impose also $\left\|h_{1}\left(P_{2}-P_{1}\right)\right\| \leq \varepsilon$.

(**) Since $F_{W}=\operatorname{Ker} d g+g^{*} F$ it inherits a natural $\operatorname{Spin}^{c}$ structure, with Clifford module $S_{W}=S_{g} \hat{\otimes} g^{*} S_{V}$ 
Let $\xi, \eta \in \mathcal{E}_{g, s_{g}}$ and $P \in \Psi_{W, s_{W^{\circ}}^{*}}^{*} \quad$ Set $P_{2}=T_{\xi}^{*} \pi_{f}(P) T_{\eta^{\circ}} \quad\left(T_{\xi}(\zeta)=\xi \otimes_{V / F} \zeta \in\right.$ $\left.\mathcal{E}_{W, S_{W}}, \zeta \in \mathcal{E}_{V, S_{V}}\right)$. Let $\Omega \subseteq W$ be a foliation chart for $W, F_{W}$. If $P$ has support in $G_{W}(\Omega)$ and $\xi, \eta$ have support in $\Omega$, then $P_{2} \in \Psi_{V, s_{V}}^{*}$ and $\sigma\left(P_{2}\right)(x, \alpha)=\int_{z \in g^{-1}(x)}$ $\left\langle\xi(z), \sigma_{p}\left(z, g^{*}(\alpha) \eta(z)\right\rangle\left(\alpha \in F_{x}^{*} ; g^{*}(\alpha) \in F_{W, z}^{*}\right)\right.$, (Lemma 1.7). This obviously remains true for general $P, \xi, \eta$.

Now since $\sigma_{Q}\left(z, g^{*}(\alpha)\right)=1_{s_{g, z}} \hat{\otimes} \sigma_{D_{V}}(g(z), \alpha)\left(z \in W, \alpha \in F_{g(z)}\right)$, we can see that $Q$ is a $D_{V}$ connection (Remark A.6.4).

Let us now check the positivity condition. We may assume that both $D_{g}$ and $D_{W}$ have supports as close to the diagonal as we wish. In particular, we may assume that each $D_{g, x}$ is diagonal in the decomposition of $g^{-1}(x)$ in connected components. In particular $F_{g} \hat{\otimes} 1=\pi_{f}\left(\widetilde{F}_{g}\right)$ where $\widetilde{F}_{g} \in \mathbb{L}\left(\mathcal{E}_{W, s_{W}}\right)$. It is now enough to prove that $h\left[\tilde{F}_{g}, D_{W}\right] \in \Psi_{0, W}^{*}$ and has non-negative symbol for $h \in C_{c}(W), h \geq 0$. As $D_{W} \in \Psi_{W}^{*},\left[h, D_{W}\right] \in \Omega\left(\mathcal{E}_{W, s_{W}}\right)$. Then we just have to compute $\left[h \widetilde{F}_{g}, D_{W}\right]$. We may now take $h$ with as small support as we wish. Hence (as the support of $\widetilde{F}_{g}$ is close to the diagonal) we may assume that there exists a foliation chart $\Omega_{W}$ and $k \in C_{c}\left(\Omega_{W}\right) \subseteq C_{c}(W)$ such that $k h=h$ and $h \widetilde{F}_{g} k=h \widetilde{F}_{g}$. Hence we now have to compute $\left[h \widetilde{F}_{g}, k D_{W} k\right]$. The whole situation is reduced to the case $V=T \times U_{2}, W=T \times U_{1} \times U_{2}, g=\operatorname{pr}_{T \times U_{1}}$ and the obvious foliation. So the answer follows from Lemmas 1.8, 1.9.

4.8. Definition. Let $W \stackrel{f}{\rightarrow} V \mid F$ be a smooth submersion. Assume that the bundle $F_{W}=d f^{-1}(F)$ defining the pull back foliation is $\operatorname{Spin}^{c}$. The equality $f !=$ $p_{W} ! \otimes_{W / F_{W}} \varepsilon_{f}$ defines the element $f ! \in K K(W, V / F)$.

The following consequence of Lemma 4.7 will allow to extend this definition to arbitrary $K$-oriented smooth maps $W \rightarrow V / F$.

49. Proposition. a) Let $X \stackrel{\pi}{\rightarrow} \underset{\mathrm{f}}{\mathrm{f}} \rightarrow V / F$ be smooth K-oriented submersions. Then $(f \circ \pi) !=\pi ! \otimes_{W} f !$.

b) Assume that the following diagram of smooth $K$-oriented maps, is commutative:

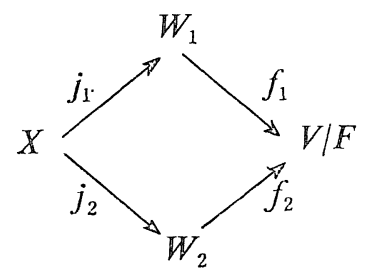

with $f_{1}, f_{2}$ submersions. Then $j_{1} ! \otimes_{W_{1}} f_{1} !=j_{2} ! \otimes_{W_{2}} f_{2} !$. 
Proof. a) By Lemma $4.7,\left(p_{W} \circ \pi\right) !=\pi ! \otimes_{W} p_{W} !$ Thus

$$
\left(p_{W} \circ \pi\right) ! \otimes \varepsilon_{f}=\pi ! \otimes_{W} p_{W} ! \otimes \varepsilon_{f}=\pi ! \otimes_{W} f ! \text { 。 }
$$

But $\left(p_{W} \circ \pi\right) !=p_{X} ! \otimes \varepsilon_{p_{W} \circ \pi} !$ so that $\left(p_{W} \circ \pi\right) ! \otimes \varepsilon_{f}=p_{X} ! \otimes \varepsilon_{f^{\circ} \pi} \stackrel{\text { def }}{=} p_{f^{\circ} \pi} !$ (by Proposition 4.4). Hence $(f \circ \pi) !=\pi ! \otimes_{W} f !$.

b) From Theorem 2.13 and a) above it is enough to construct $W$ and smooth $K$-oriented maps making the following diagram commute:

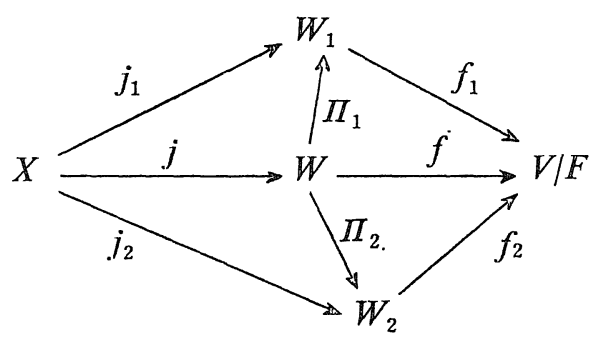

where $\Pi_{1}, \Pi_{2}, f$ are submersions.

Let us construct $W$. It is roughly speaking the fibered product of $W_{1}$ and $W_{2}$ over $V / F$. More precisely let $G_{f}=\left\{\left(\alpha_{1}, \alpha_{2}\right) \in G_{f_{1}} \times G_{f_{2}}, s_{f_{1}}\left(\alpha_{1}\right)=s_{f_{2}}\left(\alpha_{2}\right)\right\}=$ $G_{f_{1}} \times{ }_{V} G_{f_{2}}$. It is a manifold and the diagonal action of $G$ on the right makes it a principal $G$-bundle over $W=G_{f} / G$. The projections $\pi_{i}: G_{f} / G \rightarrow G_{f_{i}} / G$ are the obvious ones and they are submersions.

The commutativity of the diagram means that there exists an isomorphism $\theta$ of $G$-bundles of $j_{1}^{*}\left(G_{f_{1}}\right)$ with $j_{2}^{*}\left(G_{f_{2}}\right)$. One then defines the map $j: X \rightarrow W$, by $j(x)=\left(\alpha_{1}, \alpha_{2}\right)$ if $r_{f_{1}}\left(\alpha_{1}\right)=j_{1}(x), r_{f_{2}}\left(\alpha_{2}\right)=j_{2}(x)$ and $\alpha_{2}=\theta\left(\alpha_{1}\right)$.

4.10. Definition. Let $X \stackrel{g}{\rightarrow} V / F$ be a smooth $K$-oriented map, then $g ! \in$ $K K(X, V / F)$ is defined as any Kasparov product $j ! \otimes f !$ for any factorization $g=f \circ j$ of $g$ through a K-oriented submersion $f: W \rightarrow V / F$.

(The existence of such a factorization is proven, using the transverse microbundle (cf. [13]) in [10], §11.)

4.11. Theorem. For $X \stackrel{g}{\rightarrow} V / F$ the element $g ! \in K K(X, V \mid F)$ only depends upon the K-oriented homotopy class of $g$. For $Y \stackrel{h}{\rightarrow} X K$-oriented, one has $(g \circ h) !=h ! \otimes_{X} g !$.

Proof. One may assume that the homotopy $g_{t}$ between $g_{0}$ and $g_{1}$ is smooth. Consider it as a smooth map $X \times[0,1] \stackrel{g}{\rightarrow} V \mid F \times[0,1]$ then $g ! \in K K(X \times[0,1]$, 
$V / F \times[0,1])$ defined as in Definition 2 , gives by restriction $([0,1] \rightarrow \mathrm{pt})$ an element $\alpha \in K K(X, V / F \times[0,1])$ which is a homotopy between $g_{0}$ ! and $g_{1} !$. The second assertion follows from Theorem 2.6, together with Proposition 4.8.

Recall that the geometric group $K_{*, \tau}(B G)$ was defined in [10], as equivalence classes of cycles $(M, E, f)$, where $M$ is a compact smooth manifold, $E$ is a bundle over $M$, and $f: M \rightarrow V / F$ is smooth and $K$-oriented ${ }^{(*)}$

4.12. Corollary. The element $\mu(M, E, f): f !(E)=[E] \otimes_{M} f ! \in K^{*}(V / F)$, depends only upon the equivalence class of the cycle $(M, E, f)$.

Proof. The invariance under bordism follows from Proposition 3.5. Let $(\tilde{M}, \widetilde{E}, p \circ f)$ be a vector bundle modification of $(M, E, f)$. One has by hypothesis $p ![\widetilde{E}]=[E]$ hence the equality follows from Theorem 4.10 .

In [10] the analytical element associated to a geometric cycle was constructed in a different manner. We shall now check that this other construction gives the same result.

The construction of [10] used a Kasparov bimodule associated to an étale map $W \rightarrow V / F$; we first note that it coincides exactly with our $\mathcal{E}_{f}$, which (since $F_{W}=0$ ), is by Definition 4.6 equal to $f$ !.

4.13. Proposition. Let $X \stackrel{f}{\rightarrow} V / F$ be a K-oriented smooth map. Then for any factorisation $f=p \circ e \circ j^{(* *)}$ :

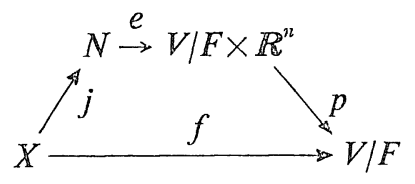

where $N$ is a manifold, $e$ is étale, and $p$ is the obvious projection, one has $f !=j !$ $\bigotimes_{N} e ! \otimes_{\mathbb{R}^{n}} \beta^{-1}$ where $\beta^{-1} \in K K\left(\mathbb{R}^{n}, \mathrm{pt}\right)$ is the Bott element ([19], §5).

Proof. We may assume that $X=N$ since $j ! \otimes(p \circ e) !=f !$ (Definition 4.8). Let us consider the two maps $g_{0}, g_{1}$ from $N \times \mathbb{R}^{n}$ to $V / F \times \mathbb{R}^{n}, g_{0}(n, t)=(p \circ e(n)$, $t), g_{1}(n, t)=e(n)$. One has $(p \circ e) ! \otimes_{C} \mathrm{id}_{R^{n}}=g_{0}$ ! (applying Definition 4.7), $g_{1} !=$ $\beta^{-1} \otimes_{C} e$ !. As there is an obvious homotopy between $g_{0}$ and $g_{1}$, one has $g_{0} !=$ $g_{1}$ !. Hence using the Bott periodicity, one gets $(p \circ e) !=e ! \otimes_{\boldsymbol{R}^{n}} \beta^{-1}$.

Specializing to the case when $X=V, N$ is the normal bundle of $F$ in $\mathbb{R}^{n}$ for

(*) For a more precise definition of the topological $K$-theory of a foliation, cf. [5].

(**) $e$ being étale is $K$-oriented. Hence $K$-orientations for $f$ and $j$ are in 1-1 correspondence. 
some embedding $V \rightarrow \mathbb{R}^{n}$, and $j: V \rightarrow N$ the obvious inclusion one gets for arbitrary foliations the precise analogue of the index theorem for families of Atiyah and Singer [2].

4.14. Theorem. Let $(V, F)$ be a smooth foliation, $P$ a longitudinal elliptic pseudo-differential operator. Let $i: V \rightarrow \mathbb{R}^{n}$ be an embedding, $N_{x}=\operatorname{di}\left(F_{x}\right)^{\perp}\left(\right.$ in $\left.\mathbb{R}^{n}\right)$ the normal bundle to $F$. Considering $N$ as an open transversal to the foliation of $V \times \mathbb{R}^{n}$ by $F \times\{0\}$ (cf. [10]), one gets a map: $\operatorname{Ind}_{t}: K(N) \rightarrow K\left(V / F \times \mathbb{R}^{n}\right) \approx K(V / F)$ (through the Bott periodicity for $C^{*}$-algebras). Then:

$$
\operatorname{Ind}_{a}(P)=\operatorname{Ind}_{t}(\tilde{\sigma}(P))
$$

where $\tilde{\sigma}(P)$ is associated to $\sigma(P) \in K^{*}\left(F^{*}\right)$ through the Thom isomorphism $K^{*}\left(F^{*}\right)$ $\approx K^{*}(N)^{(*)}$.

Here the analytical index $\operatorname{Ind}_{a}(P)$ is described by the Kasparov bimodule $\left(\mathcal{E}_{V, E}, P\right)$. It is of course the same as the image of the symbol in the connecting map of the six terms exact sequence corresponding to the exact sequence of Proposition 4.6. More explicitely, using a parametrix $Q, \operatorname{Ind}_{a}(P)$ is the class of the idempotent

$$
e=\left[\begin{array}{ll}
1-S_{0}^{2} & Q\left(S_{1}+S_{1}^{2}\right) \\
S_{1} P & S_{1}^{2}
\end{array}\right], e \in M_{2}\left(C^{*}(V, F)\right),
$$

where

$$
S_{0}=1-Q P, S_{1}=1-P Q \in C^{*}(V, F) .
$$

Proof. For simplicity, we only treat the case when $F$ is $\operatorname{Spin}^{c}$, then $N$ inherits a natural $\operatorname{Spin}^{c}$ structure with $S_{F} \hat{\otimes} S_{N} \approx S_{R^{n}}$. One can assume that $P$ is an order 0 Dirac operator with coefficients in $[E] \in K^{*}(V)$, so that $\operatorname{Ind}_{a}(P)=[E]$ $\otimes_{V} p_{V}$ ! where $p_{V}: V \rightarrow V / F$ is the projection. By Proposition 4.13, one has $p_{V}$ ! $=j ! \otimes_{N} e ! \otimes_{R^{n} \beta^{-1}}$, so that $\operatorname{Ind}_{a}(P)=[E] \otimes j ! \otimes_{N} e ! \otimes_{\mathbb{R}^{n} \beta^{-1}}$. Hence it is equal to Ind $_{t}(P)$ since $j ![E]=\tilde{\sigma}(P)$.

4.15. Remark. a) It is easy to deduce from the above theorem the index theorem for measured foliations of [9]. The transverse measure $\Lambda$ defines a linear map Trace from $^{0}(V / F)$ to $\mathbb{R}, \mathrm{cf}$. [8]. The composition of Trace $_{A} \circ$ Ind $_{a}$ is easily seen to be $\operatorname{Ind}_{\Lambda}(P)$ (see [9], [10]). The composition of $\operatorname{Trace}_{\Lambda} \circ \operatorname{Ind}_{t}(P)$ can be computed topologically and gives $\left\langle\operatorname{ch} P \operatorname{Td} F_{C}\right.$, [1] (cf. [9] or [10]).

b) The map $i: V \rightarrow \mathbb{R}^{n}$ of Theorem 4.14 is not required to be an imbedding, it is enough that its restriction to each leaf is an immersion. Thus the theorem

(*) The sum $F+N$ is the trivial bundle $\mathbb{R}^{n}$ so that $i_{N^{\circ}} p_{F}$ (with obvious notations) is $K$-oriented。 
still holds for transversally $C^{0}$ foliations and even for foliations whose transversals are just locally compact spaces. Formulated in this way it is even closer to the Atiyah-Singer index theorem for families.

4.16. The Euler Class. Let $(V, F)$ be a compact foliated manifold. The de Rham complex along the leaves yields a longitudinal elliptic operator. Let us compute its topological index using the zeros of a generic longitudinal vector field $X$ as a pseudotransversal $Z \stackrel{e}{\rightarrow} V / F$. By the genericity of $X, Z$ is a submanifold of $V$, let us $K$ orient the map $e$ from $Z$ to $V / F$. The derivative of $X$ yields an isomorphism $d X$ from the normal $N_{x}$ of $Z$ in $V(x \in Z)$ to $F_{x^{\circ}}$. Now, as $N \oplus T Z=\left.T V\right|_{Z}=(\tau \oplus F)_{Z}$ where $\tau$ is the transverse bundle, the above isomorphism yields a $K$-orientation of $e$.

4.17. Corollary. The index of the de Rham complex is equal to $e ![Z] \in$ $K^{0}(V / F){ }^{(*)}$

Proof. Let $\pi: F^{*} \rightarrow V / F$ be the projection of the total space of the longitudinal cotangent bundle to the space of leaves, with its natural $K$-orientation. Then $\otimes \pi !: K^{0}\left(F^{*}\right) \rightarrow K^{0}(V / F)$ is the analytical index. Let $i: Z \rightarrow F^{*}$ be the inclusion (obviously $K$ oriented) then $e=\pi \circ i$ so that (Theorem 4.10) $e !=i ! \otimes_{F^{*}} \pi !$. Thus one just has to check that $i ![Z]$ is the class in $K^{0}\left(F^{*}\right)$ of the symbol of $d+$ $\delta: \Lambda^{\mathrm{ev}} \rightarrow \Lambda^{\text {odd }}$. Put $\sigma_{t}(x, \xi)=\alpha_{t}(x, \xi)+\alpha_{t}(x, \xi)^{*}$, where for $t \in[0,1] \alpha_{t}(x, \xi)$ is the exterior multiplication by $\eta(x, \xi, t)=i(1-\operatorname{Inf}(t\|X\|, 1)) \xi+t X(x)$. This is clearly a homotopy between $i ![Z]$ and the symbol of $d+\delta$.

Let us consider a simple example. Let $\Gamma \in P S L(2, \mathbb{R})$ be the fundamental group of a compact Riemann surface $M=U / \Gamma$, ( $U$ the Poincaré disk) of genus $g \geq 2$. Let $V$ be the compact complex manifold $V=U \times{ }_{\Gamma} P_{1}(\mathbb{C})$ where $\Gamma$ acts on $P_{1}(\mathbb{C})$ by homographic transformations $(\operatorname{PSL}(2, \mathbb{R}) \subset P S L(2, \mathbb{C})$ ). Let $F$ be the foliation of $V$ dropped down from the foliation of $U \times P_{1}(\mathbb{C})$ with the leaves $U \times\{\mathrm{pt}\}$. By construction the holonomy covering of each leaf of the foliation $(V, F)$ is the Poincare disk. We shall endow the leaves with the canonical metric with constant curvature -1 .

Let us apply the above Corollary 4.17 to this situation. Let us determine the analytical index of the de Rham operator $D=d+\delta$ (from even forms to 1 -forms). In this situation the restriction of the Laplacian to even forms is lower bounded by $1 / 4\left(D^{*} D \geq 1 / 4\right)$ hence the analytical index $\operatorname{Ind}_{a}(D)$ is $-[\operatorname{Ker}$ $D^{*}$ ]. (Here 0 is isolated in the spectrum of $D D^{*}$ so that $\operatorname{Ker} D^{*}=\operatorname{Ker} D D^{*}$ is a

(*) $[Z] \in K^{0}(Z)$ is the class of the one dimensional trivial bundle. 
well defined idempotent in $\left.C^{*}(V, F)\right)$. On the other hand, the foliation $(V, F)$ is the foliation of the natural flat connection on the bundle $V \rightarrow M$, thus the fiber $S=P_{1}(\mathbb{C})$ is a closed transversal and has a well defined class $[S] \in K^{*}(V / F)$ (cf. [8], §8). Corollary 4.17 now reads:

$$
\left[\operatorname{Ker} D^{*}\right]=(2 g-2)[S] \text {. }
$$

Even though this foliation admits no holonomy invariant transverse measure (the action of $\Gamma$ on $P_{1}(\mathbb{C})$ is strongly proximal), the element [ $\operatorname{Ker} D^{*}$ ] is non-zero (as any of its multiples) in $K^{0}(V / F)$. Let us prove this. The bundle $V \rightarrow M$ has a smooth cross section $\sigma$. Hence the pushforward $x=i !(S) \in K^{0}(V)$ of the trivial bundle on the fiber $S$, is not a torsion element, since $\sigma^{*}(x)$ is the Bott element of $M$. Let $p: V \rightarrow V / F$ be the projection, then the above $[S] \in K^{0}(V / F)$ is equal to $p !(x)$. Thus the result follows from the injectivity of $p$ ! from $K^{0}(V)$ to $K^{0}(V / F)([10]$, p. 613).

\section{$\S$ V. Appendix $\mathbb{A}$ : Connections and Implicit Characterization of the Kasparov Product}

In [19], $\S 4, \mathrm{G}$. G. Kasparov defines the "intersection product" $(\mathcal{E}, F) \otimes_{D}$ $\left(\mathcal{E}^{\prime}, F^{\prime}\right)$ (where $\left.(\mathcal{E}, F) \in K K(A, D),\left(\mathcal{E}^{\prime}, F^{\prime}\right) \in K K(D, B)\right)$ assuming that the $D$ module $\mathcal{E}$ and the $B$ module $\mathcal{E}^{\prime}$ are of the form $\mathcal{E}=\mathfrak{S} \hat{\otimes} D, \mathcal{E}^{\prime}=\mathfrak{S} \hat{\otimes} B$ ( $\mathfrak{E}$ a separable Hilbert space) while $D$ has a unit. This is legitimate by G.G. Kasparov's Stabilization theorem ([18], Theorem 2). For our purposes, it is convenient to compute the Kasparov product without stabilizing.

In this appendix we introduce a notion of connection which allows us to give an implicit characterization of the Kasparov product.

Let $A, B, D$ be graded algebras ( $A$ separable $B$ and $D$ with countable approximate units).

Let $(\mathcal{E}, F) \in K K(A, D)$ and $\left(\mathcal{E}^{\prime}, F^{\prime}\right) \in K K(D, B)$. Put $\mathcal{E}^{\prime \prime}=\mathcal{E} \hat{\otimes}_{D} \mathcal{E}^{\prime}$. It is an $A, B$ bimodule.

For each $\xi \in \mathcal{E}$, let $T_{\xi} \in \Omega_{B}\left(\mathcal{E}^{\prime}, \mathcal{E}^{\prime \prime}\right)$ be given by $T_{\xi}(\eta)=\xi \hat{\otimes}_{D} \eta$. Its adjoint is given by $T_{\xi}^{*}\left(\xi_{1} \hat{\otimes} \eta_{1}\right)=\left\langle\xi, \xi_{1}\right\rangle \eta_{1}$.

A.1. Definition. Let $G \in \mathfrak{L}\left(\mathcal{E}^{\prime}\right)$ be such that $[d, G]^{(*)} \in \mathfrak{A}\left[\mathcal{E}^{\prime}\right]$ for all $d$ in $D$. An element $\tilde{G}$ of $\mathcal{L}\left(\mathcal{E}^{\prime \prime}\right)$ is called a $G$-connection on $\mathcal{E}$, if one has for any $\xi$ in $\mathcal{E}$ :

$$
\begin{aligned}
& T_{\xi} G-(-1)^{\partial \xi \partial G} \tilde{G} T_{\xi} \in \Omega\left(\mathcal{E}^{\prime}, \mathcal{E}^{\prime \prime}\right) \\
& G T_{\xi}^{*}-(-1)^{\partial \xi \partial G} T_{\xi}^{*} \tilde{G} \in \Omega\left(\mathcal{E}^{\prime \prime}, \mathcal{E}^{\prime}\right) .
\end{aligned}
$$

(*) All commutators are graded ones. 
A.2. Proposition. (a) For any $G \in \mathfrak{R}\left(\mathcal{E}^{\prime}\right)$ satisfying $[d, G] \in \mathscr{R}\left(\mathcal{E}^{\prime}\right)(\forall d \in D)$, there exist $G$ connections on $\mathcal{E}$.

(b) The space of G-connections is affine; the associated vector space is the space of 0 connections:

$\left\{\Omega \in \mathfrak{R}\left(\mathcal{E}^{\prime \prime}\right), \Omega(k \hat{\otimes} 1) \in \Re\left(\mathcal{E}^{\prime \prime}\right)\right.$ and $(k \hat{\otimes} 1) \Omega \in \mathscr{R}\left(\mathcal{E}^{\prime \prime}\right)$ for all $\left.k \in \Re(\mathcal{E})\right\}$.

(c) If $\tilde{G}$ is a G-connection and $k \in \Re(\mathcal{E})$ then $[\tilde{G}, k \hat{\otimes} 1] \in \Re\left(\mathcal{E}^{\prime \prime}\right)$.

Proof. (a) Let $P \in \mathcal{L}(\mathcal{E})$ be a projection and $\widetilde{G}$ a $G$-connection on $\mathcal{E}$. Then $(P \hat{\otimes} 1) \tilde{G}(P \hat{\otimes} 1) \in \mathfrak{R}\left(P \mathcal{E} \hat{\otimes}_{D} \mathcal{E}^{\prime}\right)$ is a $G$-connection on $P \mathcal{E}$. So that by the stabilization theorem ([18], Theorem 2) it is enough to construct a $G$-connection on $\mathcal{E}=\mathfrak{S} \hat{\otimes}_{C} \widetilde{D}$ ( $\widetilde{D}$ is obtained from $D$ by adjoining a unit which acts as the identity in $\left.\mathcal{E}^{\prime}\right)$. But then $\widetilde{G}=1_{\mathfrak{S}} \hat{\otimes}_{C} G \in \mathfrak{R}\left(\mathcal{E} \hat{\otimes}_{\tilde{D}} \mathcal{E}^{\prime}\right)=\mathfrak{R}\left(\mathfrak{E} \hat{\otimes}_{C} \mathcal{E}^{\prime}\right)$ is a $G$-connection (the set of $\xi \in \mathcal{E}$ such that conditions of Definition A.1 are satisfied is a closed $\widetilde{D}$ submodule of $\mathcal{E}$ as $[\widetilde{D}, G] \in \Re\left(\mathcal{E}^{\prime}\right)$, and contains $\left.\mathfrak{S} \hat{\otimes}_{C} \mathbb{C}\right)$. Call a connection of the form $\left(P \hat{\otimes}_{D} 1_{\mathcal{E}^{\prime}}\right)\left(1_{\mathfrak{F}} \hat{\otimes}_{C} G\right)\left(P \hat{\otimes}_{D} 1_{\mathcal{E}^{\prime}}\right)$ a Grassmann connection.

(b) It is clear that if $\widetilde{G}_{i}$ are $G_{i}$-connections, then $\widetilde{G}_{1}+\widetilde{G}_{2}$ is a $G_{1}+G_{2}$ connection (also $\widetilde{G}_{1} \cdot \widetilde{G}_{2}$ is a $G_{1} \cdot G_{2}$ connection). If $\Omega$ is a 0 connection and $\xi, \eta \in \mathcal{E}$ we have $\Omega T_{\xi} T_{\eta}^{*} \in \Re\left(\mathcal{E}^{\prime \prime}\right)$. But $T_{\xi} T_{\eta}^{*}=\theta_{\xi, \eta} \hat{\otimes} 1$ and the $\theta_{\xi, \eta}$ generate $\Re(\mathcal{E})$ as a closed subspace ([18], Definition 4). Hence $\Omega(\Re(\mathcal{E}) \hat{\otimes} 1) \subseteq \Re\left(\mathcal{E}^{\prime \prime}\right)$. In the same way $(\Re(\mathcal{E}) \hat{\otimes} 1) \Omega \subseteq \Re\left(\mathcal{E}^{\prime \prime}\right)$.

Conversely, if $\Omega(\Omega(\mathcal{E}) \hat{\otimes} 1) \subseteq \Omega\left(\mathcal{E}^{\prime \prime}\right)$ then $\left(\Omega T_{\xi}\right)\left(\Omega T_{\xi}\right)^{*} \in \Re\left(\mathcal{E}^{\prime \prime}\right)$. Hence $\Omega T_{\xi} \in \Re\left(\mathcal{E}^{\prime}, \mathcal{E}^{\prime \prime}\right)$.

(c) It is enough to prove it for $k=\theta_{\xi, \eta}$. But $\theta_{\xi, \eta} \hat{\otimes} 1=T_{\xi} T_{\eta}^{*}$.

We now formulate an implicit characterization of the Kasparov product $(\mathcal{E}, F) \otimes_{D}\left(\mathcal{E}^{\prime}, F^{\prime}\right)$

A.3. Theorem. There exists an $F^{\prime}$ connection $F^{\prime \prime}$ (of degree one) on $\mathcal{E}$ such that:

(a) $\left(\mathcal{E}^{\prime \prime}, F^{\prime \prime}\right)$ is a Kasparov bimodule.

(b) $\left[F^{\prime \prime}, F \hat{\otimes} 1\right]=P+h$ where $P \geq 0$ and

$$
h \in \mathfrak{J}=\left\{k \in \mathfrak{L}\left(\mathcal{E}^{\prime \prime}\right), k A \subset \Re\left(\mathcal{E}^{\prime \prime}\right), A k \subseteq \Re\left(\mathcal{E}^{\prime \prime}\right)\right\} .
$$

Such a connection is unique up to operatorial homotopy; the class of $\left(\mathcal{E}^{\prime \prime}, F^{\prime \prime}\right)$ in $K K(A, B)$ is the Kasparov product $(\mathcal{E}, F) \bigotimes_{D}\left(\mathcal{E}^{\prime}, F^{\prime}\right)$.

Proof. Existence: Let $G$ be an $F^{\prime}$ connection on $\mathcal{E}$. Let $E_{1}$ be the $C^{*}$ subalgebra of $\mathcal{L}\left(\mathcal{E}^{\prime \prime}\right)$ generated by $\Re(\mathcal{E}) \hat{\otimes} 1$ and $\Re\left(\mathcal{E}^{\prime \prime}\right)$. 
Let $E_{2}$ be the (separable) $C^{*}$-subalgebra of $\mathfrak{L}\left(\mathcal{E}^{\prime \prime}\right)$ generated by $\left\{G^{2}-1\right.$, $\left.G-G^{*},[G, F \hat{\otimes} 1],[G, a](a \in A)\right\}$. Let $\mathfrak{F}$ be the (separable) vector space spanned by $F \hat{\otimes} 1, G$ and $A$.

Finally put $E=\Omega\left(\mathcal{E}^{\prime \prime}\right)$.

As all elements of $E_{2}$ are 0 -connections $E_{1} \cdot E_{2} \subseteq E$. Using A.2(c) we get $\left[\mathfrak{F}, E_{1}\right] \subseteq E_{1}$.

Apply then Theorem 4 of Section 3 of [19] and get $M, N \in \mathbb{R}\left(\mathcal{E}^{\prime \prime}\right), M \geq 0$, $N \geq 0, M+N=1$ such that $M E_{1} \subseteq E, N E_{2} \subseteq E,[M, \mathfrak{F}] \subseteq E$.

Put then $F^{\prime \prime}=M^{1 / 2} F \hat{\otimes} 1+N^{1 / 2} G$. One gets easily that $\left(\mathcal{E}^{\prime \prime}, F^{\prime \prime}\right)$ is a Kasparov bimodule.

As $M \cdot E_{1} \subseteq E, M^{1 / 2}$ is a 0 -connection; as $[M, F \hat{\otimes} 1] \in E, M^{1 / 2}(F \hat{\otimes} 1)$ is also a 0 -connection. By A.2(b) $N^{1 / 2}$ is a 1 -connection. Hence $F^{\prime \prime}$ is an $F^{\prime}$ connection.

Finally $\left[F^{\prime \prime}, F \hat{\otimes} 1\right]=M^{1 / 2}[F \hat{\otimes} 1, F \hat{\otimes} 1]$ modulo $\Re\left(\mathcal{E}^{\prime \prime}\right)$. But as $2 M^{1 / 2}\left(F^{2} \hat{\otimes} 1\right)$ $=2 M^{1 / 2}$ modulo $\mathfrak{\Im}$ we get the positivity condition.

Uniqueness: Let first $G_{0}$ and $G_{1}$ be two $F^{\prime}$ connections. Let $E_{2}$ be the $C^{*}$-subalgebra of $\mathfrak{L}\left(\mathcal{E}^{\prime \prime}\right)$ generated by $\left\{G_{0}-G_{1}, G_{0}^{2}-1, G_{0}-G_{0}^{*},\left[G_{0}, F \hat{\otimes} 1\right],\left[G_{0}, a\right] a \in A\right\}$ and $\mathfrak{F}$ the subspace spanned by $F \hat{\otimes} 1, G_{0}, G_{1}, A$. Apply then [19], Theorem 4, $\S 3$ (with $E_{1}$ and $E$ as defined above). $\mathbb{P u t} F_{t}^{\prime \prime}=M^{1 / 2}(F \hat{\otimes} 1)+N^{1 / 2}\left((1-t) G_{0}+t G_{1}\right)$.

It now remains to prove that if $G$ is an $F^{\prime}$ connection satisfying (a) and (b), and if $M, N$ are constructed as above, we can join $G$ and $F^{\prime \prime}=M^{1 / 2}(F \hat{\otimes} 1)+$ $N^{1 / 2} G$ by a norm continuous path of $G$-connections satisfying (a) and (b).

Let $Q_{t}=(t M)^{1 / 2}(F \hat{\otimes} 1)+(1-t M)^{1 / 2} G$. Write $[F \hat{\otimes} 1, G]=P+h$ with $P \geq 0$ and $h \in \mathfrak{\Im}$. Put $Z_{t}=1+t^{1 / 2}(1-t)^{1 / 2} P$, and $F_{t}^{\prime \prime}=Q_{t} Z_{t}^{-1 / 2}$. One checks easily that $\left|Q_{t}\right|^{2}-Z_{t} \in \Im$.

Moreover $\left[A, Q_{t}\right] \subset \Omega\left(\mathcal{E}^{\prime \prime}\right)$; hence $\left[A, Z_{t}\right] \subset \Omega\left(\mathcal{E}^{\prime \prime}\right)$. Thus $\left[A, F_{t}^{\prime \prime}\right] \subset \Omega\left(\mathcal{E}^{\prime \prime}\right)$, and $\left|F_{t}^{\prime \prime}\right|^{2}-1=Z_{t}^{-1 / 2}\left(\left|Q_{t}\right|^{2}-Z_{t}\right) Z_{t}^{-1 / 2} \in \Im$. Also $\left[Q_{t}, Z_{t}\right] \in \Im$, so that $F_{t}^{\prime \prime}-$ $F_{t}^{\prime \prime *} \in \Im$.

We thus get that $\left(\mathcal{E}^{\prime \prime}, F_{t}^{\prime \prime}\right)$ is a Kasparov $A, B$ bimodule.

As $Q_{t}$ is a $G$-connection and $P$ is a 0 -connection, $F_{t}^{\prime \prime}$ is a $G$-connection.

Finally $[F \hat{\otimes} 1, P] \in \Im$ so that $\left[F \hat{\otimes} 1, Z_{t}^{-1 / 2}\right] \in \mathfrak{\Im}$ and hence $\left[F \hat{\otimes} 1, F_{t}^{\prime \prime}\right]=$ $Z_{t}^{-1 / 4}\left(2(t M)^{1 / 2}+(1-t)^{1 / 2} P\right) Z_{t}^{-1 / 4}+h_{t}$ where $h_{t} \in \Im_{\text {. }}$

Thus $F_{t}^{\prime \prime}$ is the desired homotopy between $F_{0}^{\prime \prime}=G$ and $F_{1}^{\prime \prime}=F^{\prime \prime}$.

Finally, if in the existence part we start with a Grassmann connection then $\left(\mathcal{E}^{\prime \prime}, F^{\prime \prime}\right)$ is the Kasparov product as defined in [19].

We need in fact a slight refinement of Theorem A.3: 
Let $(\mathcal{E}, F)$ be a Kasparov $A, D$ bimodule. Let $\mathcal{K}_{1}$ be the $C^{*}$-subalgebra of $\Re(\mathcal{E})$ generated by $[a, F], a\left(F^{2}-1\right), a\left(F-F^{*}\right)(a \in A)$ and the multiples by $A$, $F$ and $F^{*}$. Let $\varepsilon_{1}$ be the closed $D$ submodule of $\mathcal{E}$ generated by $\mathcal{K}_{1} \mathcal{E}$. Call this $\mathcal{E}_{1}$ the support of $(\mathcal{E}, F)$. It is obviously stable under the actions of $A$ and F.

Let $\left(\mathcal{E}^{\prime}, F^{\prime}\right)$ be a Kasparov $D, B$ bimodule. The refined version of Theorem A.3 is based on the following notion of connection localized on the support of $(\mathcal{E}, F)$.

A.4. Definition. An $F^{\prime}$ connection on $(\mathcal{E}, F)$ is an element $\tilde{G}$ of $\mathcal{L}\left(\mathcal{E}^{\prime \prime}\right)$ such that $T_{\xi} F^{\prime}-(-1)^{\partial \xi} \tilde{G} T_{\xi} \in \Re\left(\mathcal{E}^{\prime}, \mathcal{E}^{\prime \prime}\right)$ and $F^{\prime} T_{\xi}^{*}-(-1)^{\partial \xi} T_{\xi}^{*} \widetilde{G} \in \Re\left(\mathcal{E}^{\prime \prime}, \mathcal{E}^{\prime}\right)$ for all $\xi$ in the support of $(\mathcal{E}, F)$.

A.5. Theorem. In the above situation there exists $F^{\prime \prime} \in \mathfrak{Q}\left(\mathcal{E}^{\prime \prime}\right)$ such that:

(a) $\left(\mathcal{E}^{\prime \prime}, F^{\prime \prime}\right)$ is a Kasparov $A, B$ bimodule.

(b) $F^{\prime \prime}$ is an $F^{\prime}$ connection on $(\mathcal{E}, F)$.

(c) $a\left[F^{\prime \prime}, F \otimes 1\right] a^{*} \geq 0$ modulo $\Re\left(\mathcal{E}^{\prime \prime}\right)$ for all $a \in A$.

Such an $F^{\prime \prime}$ is unique up to norm homotopy and the class of $\left(\mathcal{E}^{\prime \prime}, F^{\prime \prime}\right)$ in $K K(A, B)$ is the Kasparov product $(\mathcal{E}, F) \otimes_{D}\left(\mathcal{E}^{\prime}, F^{\prime}\right)$.

Note that the condition (c) here is equivalent to condition (b) in Theorem A.3.

Proof. In view of Theorem A.3 we just have to prove uniqueness. But the proof of uniqueness in Theorem A.3 needs no changes to apply in the present situation.

Let us end this appendix with some remarks which are used in the text:

A.6. Remarks. (1) Let $A_{1}, A_{2}$ be separable, $B_{1}, B_{2}, D$ with countable approximate units. Let $\left(\mathcal{E}_{1}, F_{1}\right)$ be a Kasparov $A, B_{1} \hat{\otimes} D$ bimodule and $\left(\mathcal{E}_{2}\right.$, $\left.F_{2}\right)$ a Kasparov $D \hat{\otimes} A_{2}, B_{2}$ bimodule. Their Kasparov product $\left(\varepsilon_{1}, F_{1}\right) \otimes_{D}\left(\varepsilon_{2}\right.$, $F_{2}$ ) is computed using the equality:

$$
\left(\mathcal{E}_{1}, F_{1}\right) \otimes_{D}\left(\mathcal{E}_{2}, F_{2}\right)=\left(\mathcal{E}_{1} \hat{\otimes} A_{2}, F_{1} \hat{\otimes} 1\right) \otimes_{B_{1} \hat{\otimes} D \hat{\otimes} A_{2}}\left(B_{1} \hat{\otimes} \mathcal{E}_{2}, 1 \hat{\otimes} F_{2}\right)
$$

in $K K\left(A_{1} \otimes A_{2}, B_{1} \otimes B_{2}\right)$ (cf. [19], Theorem 4, §4).

(2) Let $(\mathcal{E}, F)$ be a Kasparov $A, B$ bimodule and let $\mathcal{E}_{1} \subseteq \mathcal{E}$ be the support of $(\mathcal{E}, F)$. Let $F_{1}$ be the restriction of $F$ to $\mathcal{E}_{1}$. Obviously $\left(\mathcal{E}_{1}, F_{1}\right)$ is a Kasparov $A, B$ bimodule. Moreover, the classes of $(\varepsilon, F)$ and $\left(\mathcal{E}_{1}, F_{1}\right)$ in $K K(A, B)$ coincide.

Indeed, let $\tilde{\mathcal{E}}$ be the Hilbert $B \otimes C[0,1]$ submodule of $\mathcal{E} \otimes C[0,1], \tilde{\mathcal{E}}=\{\xi ; \xi(1)$ $\left.\in \mathcal{E}_{1}\right\}$. Let $A$ act in an obvious way in $\tilde{\mathcal{E}}((a \xi)(t)=a \cdot \xi(t))$ and $\widetilde{F} \in \mathbb{R}(\tilde{\mathcal{E}})$ be given 
by $(\tilde{\mathbb{F}} \xi)(t)=F \xi(t)$.

Then $(\tilde{\mathcal{E}}, \tilde{F})$ is a homotopy between $(\mathcal{E}, F)$ and $\left(\mathcal{E}_{1}, F_{1}\right)$.

(3) Let $D$ and $G$ be as in Definition A.1. Suppose moreover that $[d, G]=0$ for all $d$ in $D$. Then $1 \hat{\otimes}_{D} G$ makes sense in $\Re_{B}\left(\varepsilon \hat{\otimes}_{D} \mathcal{E}^{\prime}\right)$ and is a $G$-connection on $\varepsilon$.

(4) Let $D$ and $G$ be as in Definition A.1. Let $\tilde{G} \in \mathbb{L}\left(\mathcal{E} \hat{\otimes}_{D} \mathcal{E}^{\prime}\right)$. If for all $\xi, \eta$ in $\mathcal{E}, T_{\xi}^{*} \tilde{G} \mathbb{T}_{\eta}-(-1)^{\partial \xi \partial G} G\langle\xi, \eta\rangle \in \Re\left(\mathcal{E}^{\prime}\right)$ and $\mathbb{T}_{\xi}^{*} \widetilde{G}^{*} \tilde{G} \mathbb{T}_{\eta}-(-1)^{\partial \xi \partial\left(G^{*} G\right)} G^{*} G\langle\xi, \eta\rangle \in$ $\Omega\left(\mathcal{E}^{\prime}\right)$, then $\tilde{G}$ is a $G$-connection on $\mathcal{E}$ (to see this compute $\left(T_{\xi} G-(-1)^{\partial \xi \partial G} \tilde{G} T_{\xi}\right)^{*}$ $\left.\left(\mathbb{T}_{\xi} G-(-1)^{\partial \xi \partial G} \tilde{G} T_{\xi}\right)\right)$.

Remark on the notation. By $\partial x$ we mean the degree of the homogeneous element $x$. Note that in A.6.4 above if $G$ is homogeneous $\partial\left(G^{*} G\right)=0$. However, the formulae of Definition $\mathbb{A} .1$ as well as those of Remark A.6.4 have to be understood as extended by bilinearity to the non-homogeneous case.

\section{§VI。 Afpendix B: $\mathbb{K}$-Orientation of Microbumdles}

The definition of $f$ ! of [10] that we used in Section 2 was given for a $K$ oriented map $f: X \rightarrow \mathbb{Y}$ where $X$ and $Y$ are smooth manifolds. In order to generalize this construction to the case where $Y$ is just a topological manifold (cf. Remark 2.10 (c)), we need the notion of $\mathbb{K}$-orientation of microbundles that we now discuss.

Let $X$ be a compact space. Recall that a microbundle $\tau$ over $X$ (real of dimension $n$ ) is given by a locally compact total space $Z$ and a pair of maps $X \stackrel{i}{\rightarrow} Z \underset{p}{\rightarrow} X$, satisfying some local triviality condition (cf. [23], Definition, p. 54) only the germ of $Z$ around $i(X)$ being of interest.

Let us first define the $\mathbb{K}$-theory $\mathbb{K}(\tau)$ of the microbundle $\tau$ :

A virtual vector bundle over $\tau$ is given by a triple $(\Omega, E, \sigma)$ where $\Omega$ is a neighbourhood of $i(X)$ in $Z, \mathbb{E}$ is a $\mathbb{Z} / 2$ graded hermitian vector bundle over $X$ and $\sigma$ is a continuous bounded section of $\mathscr{L}\left(p^{*} E\right)$ over $\Omega \backslash i(X)\left(\sigma \in C_{b}(\Omega \backslash i(X)\right.$, $\left.\mathscr{L}\left(p^{*} \mathbb{E}\right)\right)$ ) such that $\sigma(z)^{2}=1, \sigma(z)$ is of degree $1, \sigma(z)=\sigma(z)^{*}$ for all $z$ in $\Omega \backslash i(X)$.

If $\Omega^{\prime}$ is an open subset of $\Omega$ and $i(X) \subseteq \Omega^{\prime}$, we identify $(\Omega, E, \sigma)$ with $\left(\Omega^{\prime}, \mathbb{E},\left.\sigma\right|_{\Omega^{\prime} \backslash i(X)}\right)$.

The triple $(\Omega, E, \sigma)$ is called trivial if $\sigma$ extends to an element of $C_{b}\left(\Omega, \mathscr{L}\left(p^{*} E\right)\right)$.

Homotopies between triples are defined by triples relative to the microbundle $\operatorname{pr}_{X}^{*}(\tau)$ over $X \times[0,1]\left(\operatorname{pr}_{X}: X \times[0,1] \rightarrow X\right.$ is the projection).

$\mathbb{L}$ et $\mathbb{K}^{0}(\tau)$ be the abelian group of stable homotopy classes of such triples. 
By construction $K^{0}(\tau)$ is a module over $\mathbb{K}^{0}(\mathbb{X})$.

Let $T$ be a real vector bundle, $\tau=|T|$ the underlying microbundle. Then $K^{0}(T)$ is naturally isomorphic to $\mathbb{K}^{0}(\tau)$. The map from $K^{0}(|T|)$ to $\mathbb{K}^{0}(\mathbb{T})$ is described as follows: Let $(\Omega, E, \sigma)$ be a virtual vector bundle over $\tau$; choose a Euclidean structure of the vector bundle $T$ in such a way that the unit ball is included in $\Omega$. Then the restriction of $\sigma$ to the unit sphere $S T$ defines a Clifford symbol in the sense of the first section.

If $\tau_{1}$ and $\tau_{2}$ are two microbundles over $X$ let $\tau=\tau_{1} \times_{X} \tau_{2}$ be their Whitney sum ([23], page 59). There is a natural $\left(K(X)\right.$ bilinear) cup product: $K\left(\tau_{1}\right) \times$ $K\left(\tau_{2}\right) \rightarrow K(\tau)$ given by $\left[\left(\Omega_{1}, E_{1}, \sigma_{1}\right)\right] \times_{X}\left[\left(\Omega_{2}, E_{2}, \sigma_{2}\right)\right]=\left[\left(\Omega_{1} \times_{X} \Omega_{2}, E_{1} \hat{\otimes} E_{2}, \sigma\right)\right]$ where $\sigma\left(z_{1}, z_{2}\right)=M^{1 / 2} \sigma_{1}\left(z_{1}\right) \hat{\otimes} 1+(1-M)^{1 / 2} 1 \hat{\otimes} \sigma_{2}\left(z_{2}\right)$ where $M\left(z_{1}, z_{2}\right) \in[0,1], M_{1}$ is continuous, $M\left(z_{1}, z_{2}\right)=0$ if $z_{1}=i_{1}\left(p_{1}\left(z_{1}\right)\right) ; M\left(z_{1}, z_{2}\right)=1$ if $z_{2}=i_{2}\left(p_{2}\left(z_{2}\right)\right)\left(\left(z_{1}, z_{2}\right) \in\right.$ $\left.\Omega_{1} \times{ }_{X} \backslash\left(i_{1} \times i_{2}\right)(X)\right)$.

Recall that if $X$ is a finite simplicial complex, then for any microbundle $\tau$ over $X$ there exists $\tau^{\prime}$ with $\tau \times{ }_{X} \tau^{\prime}$ isomorphic as a microbundle to $X \times \mathbb{R}^{q}$ (cf. [23], Theorem 4.1).

B.H. Definition。 An element $[\sigma]$ of $\mathbb{K}^{*}(\tau)^{(*)}$ will be called a $\mathbb{K}$-orientation of $\tau$ if there exists a microbundle $\tau^{\prime}$ and $\left[\sigma^{\prime}\right] \in K^{*}\left(\tau^{\prime}\right)$ such that:

(a) $\tau \times{ }_{X} \tau^{\prime}$ is isomorphic to the trivial microbunole $\mathbb{X} \times \mathbb{R}^{n}$.

(b) $[\sigma] \times{ }_{X}\left[\sigma^{\prime}\right]=[\beta]$ where $[\beta] \in \mathbb{K}^{n}\left(X \times \mathbb{R}^{n}\right)$ is the Bott generaior.

We shall not spell out the details of the rather obvious:

B.2. Proposintion。 1. A K-orientation $[\sigma]$ of $\tau$ is a generator of $K^{*}(\tau)$ as a $K^{*}(X)$ module.

2. If $\left[\sigma_{1}\right]$ and $\left[\sigma_{2}\right]$ are two $K$-orientations of $\tau$, there exists a unit $\varepsilon \in \mathbb{K}^{0}(X)$ with $\sigma_{2}=\varepsilon \circ \sigma_{1}$.

3. Let $[\sigma] \in \mathbb{K}^{*}(\tau)$ and let $\tau^{\prime}$ be such that $\tau \times{ }_{X^{\prime}} \tau^{\prime} \simeq \mathbb{X} \times \mathbb{R}^{n}$. If $\left[\sigma^{\prime}\right] \in \mathbb{K}^{*}\left(\tau^{\prime}\right)$ satisfying (b) of Definition B.1 exists, it is unique. Moreover $\left[\sigma^{\prime}\right]$ is then a $\mathbb{K}$ orientation of ' $\tau^{\prime}$.

4. If $\left[\sigma^{\prime}\right]$ exists for some $\tau^{\prime}$ (satisfying (a) of Definition $\mathbb{B} .1$ ), il exists for any.

5. If $\left[\sigma_{1}\right]$ and $\left[\sigma_{2}\right]$ are $K$-orientations of $\tau_{1}$ and $\tau_{2}$ then $\left[\sigma_{1}\right] \times_{X}\left[\sigma_{2}\right]$ is a $\mathbb{K}-$ orientation of $\tau_{1} \times_{X} \tau_{2}$.

6. If $\tau_{1}$ and $\tau_{1} \times_{X} \tau_{2}$ are $K$-crientable so is $\tau_{2}$ 。

7. Let $f: Y \rightarrow X$ be a continuous map and $\tau$ a microbundle over $X$. Then if $\sigma$ is a K-orientation of $\tau, f^{*}(\sigma)$ is a K-orientation of $j^{*}(\tau)$.

8. Any K-orientation of $\tau$ gives an orientation of $\tau$ (using the Chern

(*) $[\sigma]$ is assumed to be homogeneous for the $\mathbb{E} / 2$ grading of $K_{n}^{* *}(\tau)$. 
character).

The most natural example of a $K$-orientation is given by a $\operatorname{Spin}^{c}$ structure (on a real vector bundle $T$ ) which in $C^{*}$-algebra terms is described as follows:

Associated to a Euclidean structure on the bundle $T$, is a bundle of complexified Clifford algebras (Cliff $\left.T_{x}\right)_{x \in X^{\circ}}$ Let $c: T_{x} \rightarrow$ Cliff $T_{x}, c(\xi)^{2}=\|\xi\|^{2}$, $c(\xi)=c(\xi)^{*}$ be the canonical embedding. The continuous sections of this bundle of $C^{*}$-algebras form a $\left(\mathbb{Z} / 2\right.$ graded) $C^{*}$-algebra, noted $\operatorname{Cliff}(X, T)$.

If $\operatorname{dim} T$ is even each Cliff $T_{x}$ is a matrix algebra; however it is not in general of the form $\mathfrak{L}\left(S_{x}\right)$ for some hermitian bundle $S$ (cf. [3], [14]). A Spin ${ }^{c} \operatorname{structure}^{2}$ on $T$ is given by an orientation of $T$ together with a bundle $S$ of irreducible Clifford modules.

If $\operatorname{dim} T$ is odd, the discussion is the same replacing Cliff $T_{x}$ by its even part.

If $S$ and $S^{\prime}$ are two $\operatorname{Spin}^{c}$ structures then $\operatorname{Hom}_{\mathrm{Cliff}}\left(S, S^{\prime}\right)$ is a complex line bundle $L$ and $S^{\prime}=S \otimes_{C} L$. Thus one gets a transitive and free action of the group of complex line bundles on the set of $\operatorname{Spin}^{c}$ structures, if any (these are $\operatorname{Spin}^{c}$ structures corresponding to a given orientation of $T$ ).

If $S$ is a $\operatorname{Spin}^{c}$ structure on the even dimensional Euclidean vector bundle $T$, first $S$ has a natural grading (given by Clifford multiplication by the orientation of $T$, cf. [14]) and the equality $\sigma(\xi)=c(\xi /\|\xi\|) \in \mathfrak{R}(S)(\xi \in T, \xi \neq 0)$ defines an element $\sigma$ of $K^{0}(T)$.

In the odd dimensional case one gets an element of $K^{1}(T)$.

B.3. Proposition. Let $T$ be a real vector bundle over $X$.

(a) The above construction associates in an injective way a K-orientation to each Spin $^{c}$ structure.

(b) If the microbundle $|T|$ admits a K-orientation, then $T$ admits a Spin ${ }^{c}$ structure.

Proof. (a) Note first that the group of line bundles is a subgroup of the group of units of $K^{0}(X)$ which is isomorphic (through the first Chern class) to $H^{2}(X, \mathbb{Z})$. This shows the injectivity.

If $T^{\prime}$ is a Euclidean bundle such that $T \oplus T^{\prime}$ is trivial, then $T^{\prime}$ is oriented; the equality $\operatorname{Cliff}\left(T \oplus T^{\prime}\right)=\operatorname{Cliff}(T) \hat{\otimes} \operatorname{Cliff}\left(T^{\prime}\right)$ shows that the Dixmier-Douady obstruction of $\operatorname{Cliff}\left(T^{\prime}\right)$ is 0 (cf. [12], Definition 10.7.14); hence $T^{\prime}$ has a Spin ${ }^{c}$ structure $S^{\prime}$. Moreover, using the action of $H^{2}(X, \mathbb{Z})$, we may assume that the Spin ${ }^{c}$ structure $S \hat{\otimes} S^{\prime}$ of $T \oplus T^{\prime}$ is the trivial one (on the trivial bundle $T \oplus T^{\prime}$ ). But the $[\sigma]$ corresponding to the trivial $\operatorname{Spin}^{c}$ structure is the Bott element.

(b) Assume that $T$ is of even dimension $2 p$. Let $T^{\prime}$ be a Euclidean 
bundle (of dimension $2 p^{\prime}$ ) such that the bundle $T \oplus T^{\prime}$ is trivial. Let $\operatorname{Cliff}(X, T)$ and Cliff $\left(X, T^{\prime}\right)$ be the Clifford algebras (graded trivially) of $\mathbb{T}$ and $T^{\prime}$. The Thom isomorphism ([3], [19], Theorem $8, \S 5)$ identifies $K^{0}(T)$ with $K_{0}(\mathrm{Cliff}(X, T)$ ) and $K^{0}\left(T^{\prime}\right)$ with $K_{0}\left(\operatorname{Cliff}\left(X, T^{\prime}\right)\right.$ ) (by Proposition B.2.8 above both $T$ and $T^{\prime}$ are oriented). Let $\left[S^{(0)}\right]-\left[S^{(1)}\right] \in K_{0}(\operatorname{Cliff}(X, T))$ and $\left[S^{\prime(0)}\right]-\left[S^{\prime(1)}\right] \in K_{0}\left(\operatorname{Cliff}\left(X, T^{\prime}\right)\right)$ be the virtual Clifford modules associated to the $K$-orientations of $T$ and $T^{\prime}$ through the Thom isomorphism. By hypothesis the product $\left(\left[S^{(0)}\right]-\left[S^{(1)}\right]\right) \otimes$ $\left(\left[S^{\prime(0)}\right]-\left[S^{\prime(1)}\right]\right)$ defines the same element of $K_{0}\left(\mathrm{Cliff}\left(X, T \oplus T^{\prime}\right)\right)$ as the standard irreducible module $C(X) \otimes S\left(\mathbb{R}^{2 p+2 p^{\prime}}\right)$ over $C(X) \otimes C$ liff $\left(\mathbb{R}^{2 p+2 p^{\prime}}\right)$. We thus get

$$
\left(\operatorname{dim} S^{(0)}-\operatorname{dim} S^{(1)}\right)\left(\operatorname{dim} S^{\prime(0)}-\operatorname{dim} S^{\prime(1)}\right)=2^{p+p^{\prime}} .
$$

As $S^{(0)}$ and $S^{(1)}$ are $\operatorname{Cliff}(X, T)$ modules, $\operatorname{dim} S^{(0)}$ and $\operatorname{dim} S^{(1)}$ are multiples of $2^{p}$. Also $\operatorname{dim} S^{\prime(0)}$ and $\operatorname{dim} S^{\prime(1)}$ are multiples of $2^{p^{\prime}}$. So that either $S^{(0)}$ or $S^{(1)}$ (say $S^{(0)}$ ) is of dimension $r 2^{p}$ where $r$ is odd.

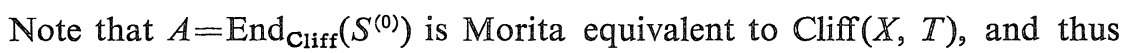
has the same Dixmier-Douady obstruction $w \in H^{3}(X, \mathbb{Z})$. For each $x \in X, A_{x}$ is a matrix algebra of dimension $r^{2}$ so that ([14]) $r w=0$. But as $T \oplus T$ is naturally Spin $^{c}$ (see Remark B.4 below) we get $2 w=0$ hence $w=0$.

If $\operatorname{dim} T$ is odd, replace $T$ by $T \oplus R$.

Note that in general $H^{2}(X, \mathscr{Z})$ is strictly contained in the group $K^{0}(X)^{-1}$ of units in $K^{0}(X)$, so that the map which to a Spin ${ }^{c}$ structure of $\mathbb{T}$ associates a $K$-orientation of $|T|$ is not surjective.

B.4. Remark. Recall that if $T$ is a real vector bundle on $X, T \oplus T$ has a natural $\mathrm{Spin}^{c}$ structure coming from its complex structure.

For a complex Hermitian bundle $T$ put $S=\Lambda(T)$ and for $\xi \in \mathbb{T}$ put $c(\xi)=$ $e_{\xi}+e_{\xi}^{*}$ where $e_{\xi}(w)=\xi \wedge w$. As $c(\xi)^{2}=\|\xi\|^{2}$ the bundle $S$ is a Cliff $T$ graded module with grading by even and odd exterior powers.

In particular if the microbundle $\tau$ is $|T|$ for some real vector bundle $T, \tau \times{ }_{X} \tau$ is $K$-oriented. It is not clear how this $K$-orientation depends upon the choice of $T$.

B.5. Defimition. Let $X$ be a smooth manifold and $Y$ a topological manifold. Let $f: X \rightarrow Y$ be a continuous map. Let $\tau_{Y}$ be the tangent microbundle of $Y$ (given by $Y \stackrel{\Delta}{\rightarrow} Y \times Y \stackrel{\mathrm{pr}_{1}}{\rightarrow} Y, \Delta(y)=(y, y)$, cf. [23], Example 3, § 2). A K-orientation of $f$ is a K-orientation of the microbundle $\tau_{f}=\left|T^{*} X\right| \times_{X} f^{*}\left(\tau_{Y}\right)$ (in the sense of Definition B.1). 
Let us gather some easy facts in the following:

B.6. Proposition. (a) If $X$ and $Y$ are $K$-oriented, any continuous map from $X$ to $Y$ is K-oriented. In particular, if $Y$ and $f$ are smooth then $d f: T X \rightarrow T Y$ is $K$-oriented.

(b) Id $d_{X}$ has a canonical K-orientation.

(c) Let $f_{1}: X_{1} \rightarrow X_{2}$ and $f_{2}: X_{2} \rightarrow Y$ be continuous maps where $X_{1}, X_{2}$ are smooth manifolds $Y$ is a topological manifold. If $f_{1}$ and $f_{2}$ are $K$-oriented then so is $f_{2} \circ f_{1}$. If $f_{2}$ and $f_{2} \circ f_{1}$ are $K$-oriented, then so is $f_{1}$.

(d) Let $Y$ be smooth and $f: X \rightarrow Y$ a smooth immersion. Let $N$ be the normal bundle of $f$. Then $f$ is $K$-orientable if and only if $N$ is $K$-orientable.

(e) Let $Y$ be smooth and $f: X \rightarrow Y$ a smooth submersion. Then $f$ is $K$ orientable if and only if the bundle $\operatorname{Ker} d f($ over $X)$ is $K$-orientable.

In this proposition, the following conventions are made:

(a) If $\sigma_{X}$ is a $K$-orientation of $T X$, we let $\sigma_{X}^{-1}$ be the $K$-orientation of $T^{*} X$ such that $\sigma_{X}^{-1} \times_{X} \sigma_{X}$ is the $K$-orientation of $T^{*} X \oplus T X$ given by the complex structure where tangent vectors are real and cotangent imaginary. The $K$ orientation of $f$ is then $\sigma_{\bar{X}}^{-1} \times_{X} f^{*}\left(\sigma_{Y}\right)$.

The $K$-orientation of $T X$ (or $T Y$ ) is given by the same almost complex structure as in [1]: horizontal vectors are real, vertical are imaginary.

(b) The $K$-orientation of $\tau_{\operatorname{Id}_{X}}$ is given by the complex structure of $T^{*} X \oplus T X$ discussed in (a).

(c) The $K$-orientations $\sigma_{1}$ of $f_{1}, \sigma_{2}$ of $f_{2}$ and $\sigma$ of $f_{2} \circ f_{1}$ are related by the equality $f_{1}^{*} \delta_{2} \times_{X_{1}} \sigma=\sigma_{1} \times_{X_{1}} f_{1}^{*} \sigma_{2}$ where $\delta_{2} \in K\left(T^{*} X_{2} \oplus T X_{2}\right)$ is the $K$-orientation of $\operatorname{Id}_{X_{2}}$ (we have identified $f_{1}^{*}\left(\tau_{\operatorname{Id}_{X_{2}}}\right) \times_{X_{1}} \tau_{f_{2^{\circ}} f_{1}}$ with $\tau_{f_{1}} \times_{X_{1}} f_{1}^{*}\left(\tau_{f_{2}}\right)$ ).

(d) The $K$-orientations $\sigma$ of $\tau_{f}$ and $\sigma^{\prime}$ of $N$ are related by the equality $\sigma=\delta_{X} \times_{X} \sigma^{\prime}$ where $\delta_{X}$ is the $K$-orientation of $\operatorname{Id}_{X}$ (we have identified $\tau_{f}$ with $\left.\tau_{\text {Id } X} \times{ }_{X} N\right)$.

(e) The $K$-orientations $\sigma$ of $\tau_{f}$ and $\sigma^{\prime}$ of $\operatorname{Ker}(d f)$ are related by the equality $\sigma=\sigma^{\prime} \times_{X} f^{*}\left(\delta_{Y}\right)$.

\section{Bibliography}

[1] M.F. Atiyah and I. Singer, The index of elliptic operators I, Ann. of Math., 87 (1968), 484-530.

[2] — The index of elliptic operators IV, Anil. of Math., 93 (1971), 119-138.

[3] M.F. Atiyah, R. Bott and Shapiro, Clifford Modules, Topology, 3, suppl. 1 (1964), 338.

[4] P. Baum, Cycles, Cocycles and $K$ theory, to appear. 
[5] P. Baum and A. Connes, Geometric $K$-theory for Lie groups and Foliations, preprint.

[6] P. Baum and R. Douglas, K-holomology and index theory, Proceedings of A.M.S., 38.

[7] L. Boutet de Montvel, A course on pseudodifferential operators and their applications, Duke Univ. Math. Series, $\mathbb{I I}$.

[8] A. Connes, An analogue of the Thom isomorphism, for crossed products of a $C^{*}$ algebra by an action of $\mathbb{R}, A d v$. in Math., 39 (1981).

[9] - Sur la théorie non commutative de l'intégration, Lecture Notes in Math., 725, (1979), Springer, 19-143.

[10] - A survey of foliations and operator algebras, Proceedings of the A.M.S., 38, 521-628.

[11] A. Connes et G. Skandalis, Théorème de l'indice pour les feuilletages, C.R. Acad. Sci. Paris, 292 (1981), 871-876.

[12] J. Dixmier, Les $C^{*}$-algèbres et leurs représentations 2ème édition, Paris Gauthier Villars, 1969.

[13] A. Haefliger, Feuilletages sur les variétés ouvertes, Topology, 9 (1970), 183-194.

[14] J. Hayden and R.J. Plymen, On the invariant of Dixmier-Douady, preprint.

[15] M.W. Hirsh, Differential topology, Graduate texts in Math. 33, Springer Verlag, Berlin.

[16] L. Hörmander, On the index of pseudodifferential operators, Elliptische Differentialgleischungen Band II, Koll., Berlin, 1969.

[17] G.G. Kasparov, Topological invariants of elliptic operators. I: $K$ homology, Math. USSR Izv., 9 (1975), 751-792.

[18] - Hilbert $C^{*}$-modules: Theorems of Stinespring and Voiculescu, Journal of operator theory, 4 (1980).

[19] - Operator $K$ functor and extensions of $C^{*}$-algebras, Izv. Akad. Nauk, S.S.S.R. ser. Mat., 44 (1980), 571-636.

[20] - $K$-theory, group $C^{*}$-algebras and higher signatures (Conspectus), part $1-2$, preprint.

[21] J.M. Kister, Microbundles are bundles, Ann. of Math., 80 (1964), 190-199.

[22] B. Lawson, Foliations, Bull. Am. Math. Soc., 80 (1974), 369-418.

[23] J. Milnor, Microbundles I, Topology, 3, Suppl. 1 (1964), 53.

[24] R.S. Palais, Seminar on the Atiyah-Singer index theorem, Annals of Math. Studies, 57 (1965).

[25] I. Singer, Future extensions of index theory and elliptic operators, Prospects in Math., Annals. of Math. Studies, 70.

[26] E. Winkelnkemper, The graph of a foliation, preprint. 
